JORGE CARVALHO COSTA

\title{
ANÁLISE LINEAR DE CASCAS COM MÉTODO DE GALERKIN LIVRE DE ELEMENTOS
}

Dissertação apresentada à Escola Politécnica da Universidade de São Paulo para obtenção do título de Mestre em Engenharia 


\title{
JORGE CARVALHO COSTA
}

\section{ANÁLISE LINEAR DE CASCAS COM MÉTODO DE GALERKIN LIVRE DE ELEMENTOS}

\author{
Dissertação apresentada à Escola Politécnica \\ da Universidade de São Paulo para obtenção \\ do título de Mestre em Engenharia \\ Área de Concentração: \\ Engenharia de Estruturas
}

Orientador:

Prof. Dr. Paulo de Mattos Pimenta 
Este exemplar foi revisado e alterado em relação à versão original, sob responsabilidade única do autor e com a anuência de seu orientador.

São Paulo, 8 de outubro de 2010.

Assinatura do autor

Assinatura do orientador

FICHA CATALOGRÁFICA

Costa, Jorge Carvalho

Análise linear de cascas com método de Galerkin livre de elementos / J.C. Costa. -- ed.rev. -- São Paulo, 2010.

$98 \mathrm{p}$.

Dissertação (Mestrado) - Escola Politécnica da Universidade de São Paulo. Departamento de Engenharia de Estruturas e Geotécnica.

1. Cascas (Engenharia) 2. Método sem malha 3. Método de Galerkin I. Universidade de São Paulo. Escola Politécnica. Departamento de Engenharia de Estruturas e Geotécnica II. t. 
A meus pais, meus grandes incentivadores, meus grandes exemplos. 


\section{AGRADECIMENTOS}

Primeiramente, agradeço a Deus por me proporcionar as melhores condições para realização deste trabalho com saúde e paz.

Muitas pessoas merecem meu agradecimento. Antes de cometer qualquer injustiça ao não citar nomes, agradeço a todos aqueles que contribuíram direta ou indiretamente para a realização deste trabalho.

Agradeço imensamente ao Prof. Paulo Pimenta pelo apoio, o incentivo, o conhecimento e o entusiasmo transmitidos ao longo do desenvolvimento desse trabalho. Estendo ao Prof. Carlos Tiago a mesma gratidão por me acolher como aluno, me receber para o estágio em Lisboa e pelo continuado apoio. Ao Prof. Eduardo Campello pela atenção dispensada nesses estudos.

A todos os amigos que fiz na Escola Politécnica e no IST, nos quais tomo a liberdade de incluir esses professores e os colegas Henrique (e Mônica), Marcelo, Eduardo, Evandro, Fernando, Leonardo, Paulo Nigro, Luís, Cínthia, Márcia e todos os outros que me fizeram sentir em casa, me apoiaram e incentivaram. Obrigado pela excelente companhia.

Aos amigos que, dentro ou fora da Engenharia, aguentaram meu lamentar de que "a matriz tá dando singular". A Walter, Ryan, Thaísa e Rita, por manterem os retirantes sempre juntos. A Marcelinho, Samuel, Higor Sérgio, Ariel, Marcel, Anísia, Gustavo, Juliana, Jorge, Fábio, Lucas, Diogo e todos os outros amigos cujos nomes esse acanhado espaço não permite citar, mas que me fazem sentir bem em qualquer lugar, especialmente em Aracaju. A Alexandre, pela amizade e a paciência no conviver.

A toda minha família. Qualquer agradecimento que possa fazer a vocês é pouco. A meus avôs Joaldo e Lealdo que por mim intercedem de um plano superior. A minhas avós Iza e Inês, que por mim pedem em suas preces e que tão bem me recebem. Meus avós "emprestados" Eunice e Osvaldo e toda sua família, que adotei como minha também. A minhas tias, em especial Têca, Lica, Rosa e Ângela, minhas mães, pelo amor que me têm. Tia Nana, também mãe, mas com o carinho e incentivo especial de "companheira acadêmica". A meus tios, sempre exemplos para mim. Tios João, Joca, Bôsco e Clovis. A Thiago e seus amigos que me receberam em São Paulo e tornaram a adaptação bem mais divertida. A meus primos Joaldo, Evelyne e Izinha, por assumirem os postos de meus irmãos. A meus primos/afilhados Vítor, Matheus, Rodrigo, Eduardo, Thainá e Geovana, por aguentarem minhas brincadeiras.

Por fim, especiais agradecimentos a meu pai, Jorge Lima e minha mãe Luzia. Vocês são meus exemplos, meus modelos. Vocês são responsáveis pelo que eu faça de bom. Obrigado pelo apoio, por entender minha ausência e por me ajudarem em todos os sentidos possíveis.

Agradeço também à FAPESP pelo apoio financeiro na forma de bolsa de mestrado (processo 2009/04525-5) e à CAPES por bolsa concedida anteriormente. 


\section{RESUMO}

O Método dos Elementos Finitos é a forma mais difundida de análise estrutural numérica, com aplicações nas mais diversas teorias estruturais. Contudo, no estudo das cascas e alguns outros usos, suas deficiências impulsionaram a pesquisa em outros métodos de resolução de Equações Diferenciais Parciais. O presente trabalho utiliza uma dessas alternativas, o Método de Galerkin Livre de Elementos (Element-Free Galerkin) para estudar as cascas. Inicia com a observação da aproximação usada no método, os Moving Least Squares e os Multiple-Fixed Least Squares. A seguir, estabelece uma formulação que combina a teoria de placas moderadamente espessas de Reissner-Mindlin à teoria da Elasticidade Plana e se utiliza da aproximação estudada para analisar placas e chapas deste tipo. Depois, expõe uma teoria geometricamente exata de cascas inicialmente curvas onde as curvaturas iniciais são impostas como deformações livres de tensão a partir de uma configuração de referência plana. Tal teoria exclui a necessidade de coordenadas curvilíneas e consequentemente da utilização de objetos como os símbolos de Cristoffel, já que todas as integrações e imposições são feitas na configuração plana de referência, em um sistema ortonormal de coordenadas. A imposição das condições essenciais de contorno é feita por forma fraca, resultando em um funcional híbrido de deslocamentos que permite a maleabilidade necessária ao uso dos Moving Least Squares. Esse trabalho se propõe a particularizar tal teoria para o caso de pequenos deslocamentos e deformações (linearidade geométrica), mantendo a consistência das definições de tensões e deformações generalizadas enquanto permite uma imposição da forma fraca resultante, depois de discretizada, por um sistema linear de equações. Por fim, exemplos numéricos são usados para discutir sua eficácia e exatidão.

Palavras-chave: Cascas. Método sem Malha. Método de Galerkin. 


\begin{abstract}
The Finite Element Method is the most spread numerical analysis tool, applied to a wide range of structural theories. However, for the study of shells and other problems, some of its deficiencies have stimulated research in other methods for solving the derived Partial Differential Equations. The present work uses one of those alternatives, the Element Free Galerkin Method, for the study of shells. It begins with the observation of the approximation used in the method, Moving Least Squares and Multiple-Fixed Least Squares. Then, it establishes a formulation that combines the Reissner-Mindlin moderately thick plate theory with plane elasticity, and uses the proponed approximation to analyze such plates and stabs. Afterwards, it demonstrates a geometrically exact shell theory that accounts for initial curvatures as a stress-free deformation from a flat reference configuration. Such theory precludes the use of curvilinear coordinates and, subsequently, the use of objects such as Cristoffel symbols, as all integrations and impositions are done in the flat reference configuration, in an orthogonal frame. The essential boundary conditions are imposed in a weak statement, rendering a hybrid displacement functional that provides the necessary conditions for the use of Moving Least Squares. This work's main objective is the particularization of this theory for the small displacement and strains assumption (geometrical linearity), keeping the consistent definition of generalized stresses and strains, while allowing the imposition of the discretized weak form through a system of linear equations. Lastly, numerical simulations are carried out to assess the method's efficiency and accuracy.
\end{abstract}

Key-words: Shells. Meshless Methods. Galerkin Methods. 


\section{SUMÁRIO}

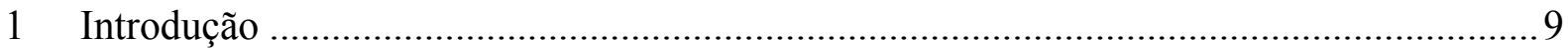

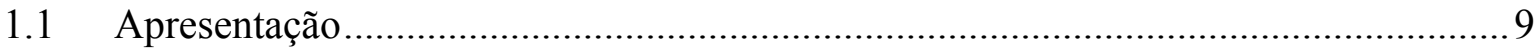

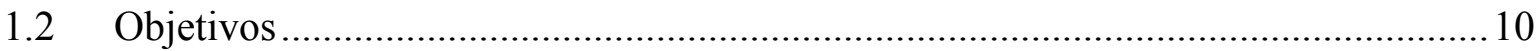

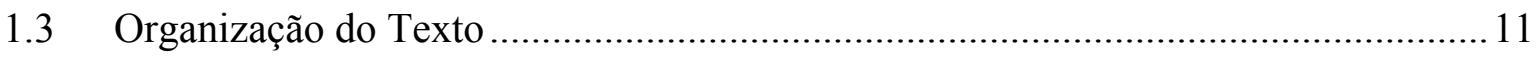

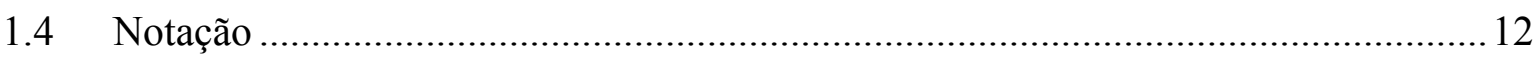

2 Aproximação por Mínimos Quadrados ................................................................... 13

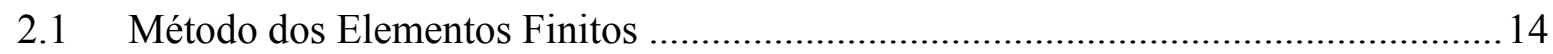

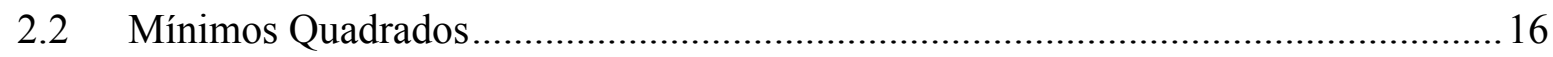

2.3 Distance-Weighted Least Squares ou Moving Least Squares ............................... 18

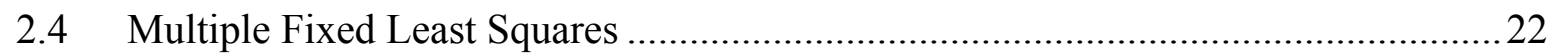

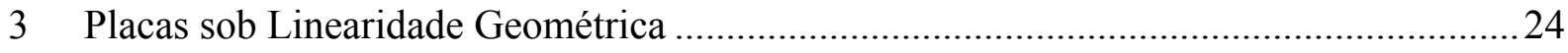

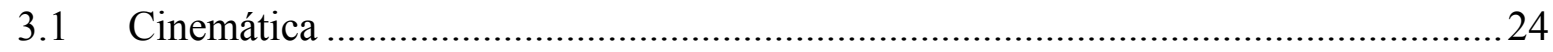

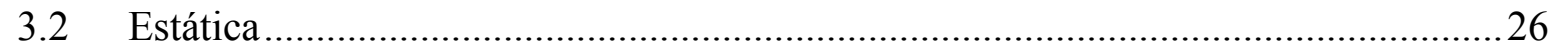

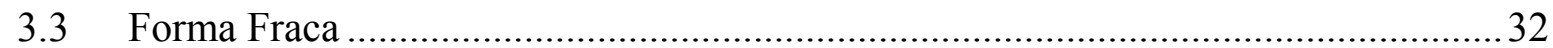

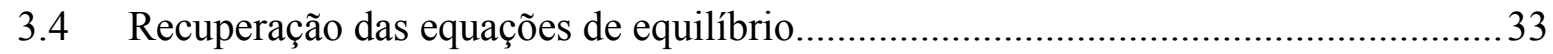

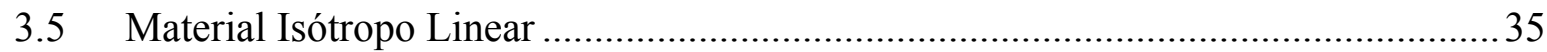




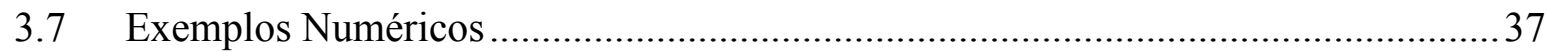

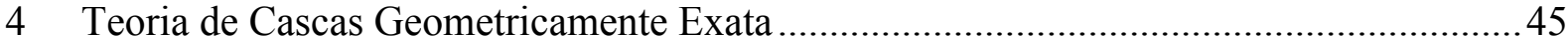

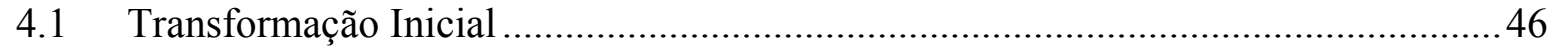

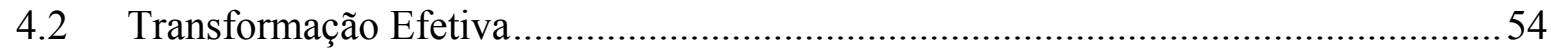

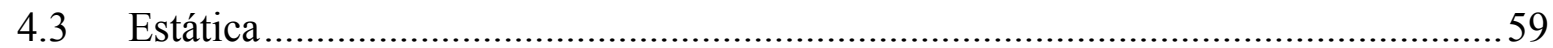

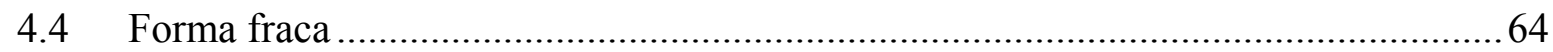

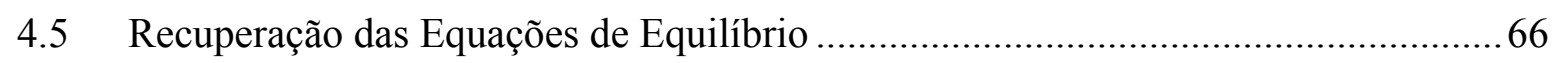

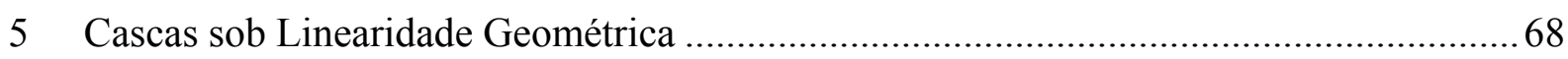

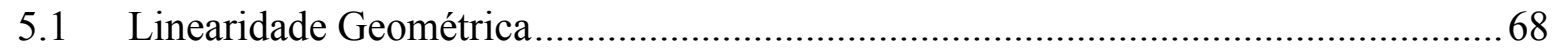

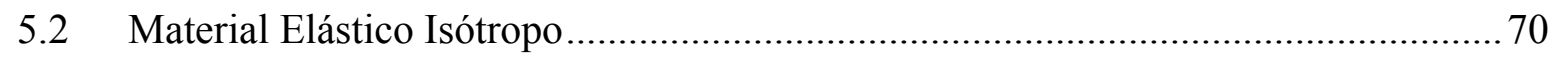

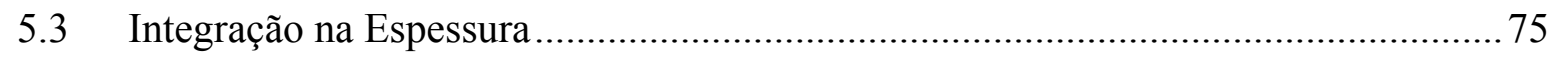

5.4 Implementação em Método de Galerkin Livre de Elementos ................................. 79

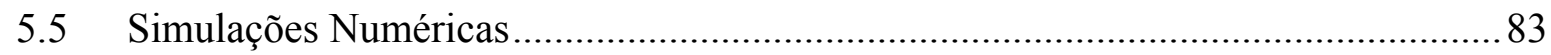

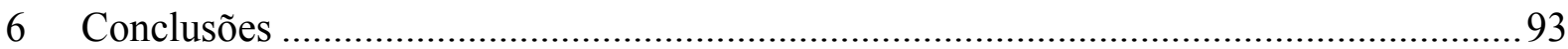

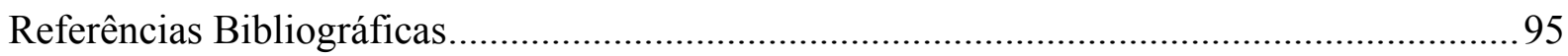




\section{INTRODUÇÃO}

\subsection{Apresentação}

Há cerca de meio século o Método dos Elementos Finitos vem sendo desenvolvido e aprimorado, tornando-se o processo de aproximação usado na maior parte das simulações numéricas, tanto para estruturas quanto em outros campos de aplicação. Esse extenso desenvolvimento permite que exista hoje uma vasta gama de aplicações para o mesmo e que seus problemas e dificuldades sejam bem conhecidos e, na medida do possível, contornados.

Contudo, algumas adversidades persistem, como deterioração da precisão quando de grandes distorções de malha, necessidade de uma malha bastante refinada para análise de problemas com grandes gradientes, distorção da homogeneidade e da isotropia causada pela orientação da malha e principalmente, dificuldade de se obter continuidades nas aproximações.

Segundo Li e Liu [3] (com tradução nossa),

Na maior parte das aplicações, os campos de interpolação do MEF são
primordialmente funções $C^{0}(\Omega)$. Campos de interpolação de ordem superior são
difíceis de construir para domínios de geometria arbitrária em múltiplas dimensões.
Isto restringe severamente a capacidade da computação baseada em Elementos
Finitos de ser utilizada em problemas práticos com derivadas de altas ordens, como
simulações de placas e cascas, elasticidade e plasticidade de grandes gradientes.

Essa deficiência provocou a busca de métodos de aproximação que propiciassem uma maior continuidade dos resultados obtidos, que levaram aos métodos sem malha. "[os métodos de partículas sem malhas] podem facilmente prover um campo de interpolação de maior grau de continuidade em qualquer dimensão, o que torna fácil a implementação de formulações de única variável ou mistas pelo método de Galerkin”. (Li e Liu [3]).

O presente trabalho busca utilizar algumas dessas vantagens para análise de cascas. Para tal, se utilizará da teoria de cascas inicialmente curvas desenvolvida por Pimenta e Campello em [1], empregando aproximações por Mínimos Quadrados Móveis (Moving Least Squares) para 
os campos de aproximação e teste, como feito por Tiago [2]. O presente trabalho será, então, uma particularização do último ao apresentar uma linearização da teoria ali contida, mediante a consideração de pequenos deslocamentos generalizados.

A referida teoria parte de uma configuração plana de referência para a casca, a partir da qual a mesma é mapeada para a sua configuração inicial curva. A partir desta é que as deformações efetivas ocorrem. Essa estratégia torna desnecessário o uso de métricas de geometria diferencial e usa apenas sistemas de coordenadas ortogonais, excluindo-se de recursos como os símbolos de Christoffel. Também é totalmente baseado em imposições cinemáticas, e não em sólidos degenerados.

Assim, possui as características de

(i) esforços e deformações generalizadas são definidas de uma forma totalmente consistente, gerando uma teoria completa em resultantes de tensão e (ii) deformações de cisalhamento de primeira ordem encontram-se incluídas no modelo, já que uma cinemática do tipo Reissner-Mindlin é considerada, baseada em um diretor inextensível (sem variação de espessura). (Pimenta e Campello [1], tradução nossa).

As deformações efetivas generalizadas são então tomadas em sua primeira ordem nos deslocamentos e rotações efetivas da casca e o material é particularizado para o caso linear.

A forma fraca obtida no processo se assemelha àquela presente em Belytschko et al. [15], mesmo partindo-se de formulações diferentes. Como descrito nesse trabalho, a combinação dessa forma fraca com o uso dos Moving Least Squares para os campos de aproximação e teste, aqui utilizada constitui um Método de Galerkin Livre de Elementos (Element-Free Galerkin Method).

\subsection{Objetivos}

O presente trabalho busca desenvolver o uso de Métodos Numéricos Sem Malha na análise linear de cascas, incluindo as inicialmente curvas. Para tal, utilizará a teoria geometricamente 
exata apresentada em Pimenta e Campello em [1] e Tiago [2], impondo as restrições da teoria linear de Reissner-Mindlin.

O resultado será uma teoria de primeira ordem nos deslocamentos e rotações que mantem as definições consistentes das deformações generalizadas, bem como dos esforços generalizados.

Também pretende utilizar o Método de Galerkin Livre de Elementos (Element-Free Galerkin Method), elaborado a partir da aproximação por Mínimos Quadrados Móveis (Moving Least Squares) para a aproximação dos deslocamentos e rotações da casca e uma interpolação das reações da fronteira cinemática por polinômios lineares de Lagrange. Tal procedimento transformará o funcional híbrido resultante da teoria linearizada em um sistema de equações linear nos deslocamentos, nas rotações e nas reações generalizadas da fronteira cinemática.

Por fim, apresentará exemplos numéricos que provam as habilidades do método, comparandoos com resultados obtidos previamente por outros pesquisadores e outros métodos.

\subsection{Organização do Texto}

Além deste capítulo introdutório, este texto possui outros cinco. No capítulo 2 é feita uma pequena explanação sobre a aproximação por mínimos quadrados móveis. No capítulo 3 , apresenta-se uma unificação das teorias de placa e chapa para superfícies planas, com o objetivo de se obter uma base a ser utilizada para descrever as cascas. Exemplos numéricos obtidos com a aproximação dos deslocamentos e rotações com Moving Least Squares são expostos.

No capítulo 4, a mencionada teoria de cascas inicialmente curvas é apresentada em sua forma geometricamente exata. No capítulo seguinte, é particularizada para o caso de pequenas deformações na transformação efetiva, descreve-se o processo de discretização da casca com Moving Least Squares e são apresentados exemplos numéricos que ilustram o desempenho do modelo apresentado.

O capítulo 6 reúne as conclusões pertinentes ao trabalho. 


\subsection{Notação}

Ao decorrer do texto, letras minúsculas itálicas, gregas ou latinas $(a, b, \ldots, \alpha, \beta, \ldots)$ representam escalares, letras minúsculas itálicas e negritas, gregas ou latinas $(\boldsymbol{a}, \boldsymbol{b}, \ldots, \boldsymbol{\alpha}, \boldsymbol{\beta}, \ldots)$ representam vetores e letras maiúsculas itálicas e negritas, gregas ou latinas $(\boldsymbol{A}, \boldsymbol{B}, \ldots)$ representam tensores de segunda ordem no espaço vetorial Euclidiano. A convenção da somatória para índices repetidos é adotada, com índices gregos variando de $1 \mathrm{a}$ 2 e índices latinos de 1 a 3. 


\section{APROXIMAÇÃO POR MÍNIMOS QUADRADOS}

Seja o problema aproximar uma função $u(\boldsymbol{x})$ em um domínio $\Omega$. Essa necessidade pode vir das mais diversas áreas, sendo, na opinião do autor, as que mais contribuíram para o desenvolvimento científico, a extensão de valores conhecidos em determinados pontos para posições onde não se conhece a função (scattered data fitting) e a resolução de problemas elípticos de valor de contorno.

Várias soluções foram propostas para esse tipo de problema ao longo do tempo. Esse texto se preocupa com duas das mais usadas na resolução de problemas estruturais: primeiro apresenta a interpolação por Elementos Finitos, que servirá de base para melhor entendimento da técnica seguinte, que dispensa o uso de uma malha e se utiliza apenas de pontos, ou partículas, para fazer a aproximação.

Dentre as soluções livres de malhas, pode-se destacar (em seus nomes originais em inglês, a fim de evitar traduções faltosas e dificuldade para futuras consultas) as Radial Basis Functions, os Kernel Particle Methods, entre eles o Reproducing Kernel Particle Method, derivado da Smoothed Particle Hydrodynamics, as Extrinsically Nodal Enricheted Approximations, onde se incluem as hp-clouds, os Natural Neighbor Interpolants e os esquemas de Least Squares (Mínimos Quadrados). Os últimos englobam os Moving Least Squares, escolhidos para serem usados nos capítulos seguintes.

Este método foi inicialmente estudado para a solução de problemas de Elasticidade Plana por Nayroles et al. [20], onde recebia o nome de Diffuse Approximation. Contudo, segundo Belytschko et al. [15], já havia sido estudado para aproximação de dados dispersos por Lancaster e Šalkauskas, McLain, Gordon e Wixom, Barnhill entre outros e recebia a denominação de Moving Least Squares. Em Tiago [2], são inicialmente chamados de Distance-Weighted Least Squares e logo identificados como Mínimos Quadrados Móveis.

Tanto a aproximação por Elementos Finitos quanto os Moving Least Squares se utilizam de um conjunto de pontos $\overline{\boldsymbol{x}}$, chamados de nós ou partículas, que "acomodarão" valores para a 
aproximação, de forma que em qualquer ponto do domínio será obtida a partir destes valores. Pode-se referir a eles como valores ou parâmetros nodais $\overline{\boldsymbol{u}}$, sendo $\bar{u}_{i}$ esse valor em cada ponto $\overline{\boldsymbol{x}}_{i}$.

Também em ambos os casos, o valor da aproximação $u^{h}$ será dado por uma combinação linear de funções definidas no domínio, chamadas de funções de forma $\Phi$. Os coeficientes desta combinação linear serão os parâmetros nodais $\overline{\boldsymbol{u}}$, de forma que

$$
u^{h}(\boldsymbol{x})=\sum_{i=1}^{n} \Phi_{i} u_{i}=\boldsymbol{\Phi}(\boldsymbol{x}) \overline{\boldsymbol{u}} .
$$

\subsection{Método dos Elementos Finitos}

O método dos elementos finitos usa uma aproximação baseada em elementos para descrever a geometria e as grandezas incógnitas do problema (na sua formulação clássica, os deslocamentos). Tome-se, por exemplo, a Figura 1, onde o domínio foi dividido em três elementos ( $e 1, e 2, e 3)$, os dois primeiros contendo dois nós cada, e o terceiro contendo 3 nós.

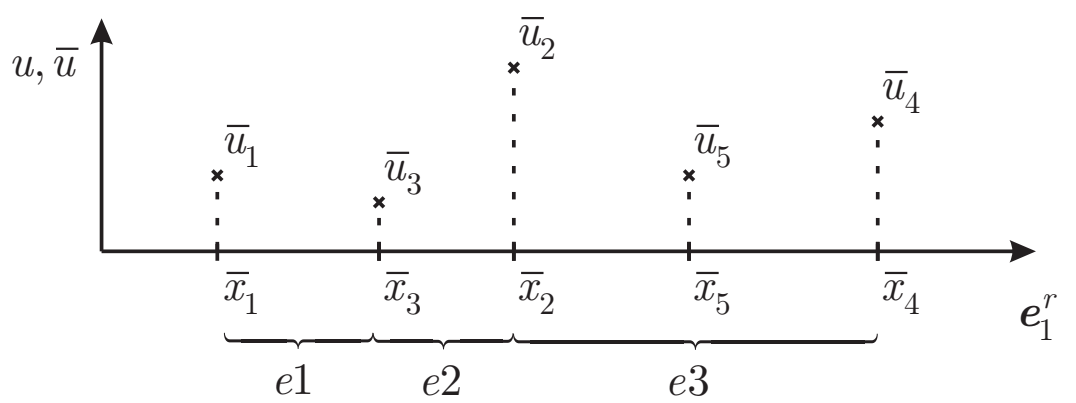

Figura 1 - Divisão do domínio em elementos

A aproximação tem, então, a forma

$$
u^{e}(\boldsymbol{x})=\boldsymbol{p}(\boldsymbol{x}) \cdot \boldsymbol{a}^{e},
$$

onde $u^{e}(\boldsymbol{x})$ é o valor da aproximação no ponto $\boldsymbol{x}$ dentro do elemento $e ; \boldsymbol{p}(\boldsymbol{x})$ é um vetor de funções, normalmente polinômios, definidas no elemento $e$, chamado base; e $\boldsymbol{a}^{e}$ é o 
conjunto de parâmetros para esse elemento. Dado o conjunto $\overline{\boldsymbol{x}}$ de pontos onde os valores dessa aproximação são definidos $(\overline{\boldsymbol{u}})$, podemos escrever

$$
\bar{u}=\boldsymbol{P}(\overline{\boldsymbol{x}}) \boldsymbol{a}^{e},
$$

onde cada linha de $\boldsymbol{P}(\overline{\boldsymbol{x}})$ é a base da aproximação $\boldsymbol{p}$ avaliada no ponto $\overline{\boldsymbol{x}}_{i}$.

Se o número de componentes da base $\boldsymbol{p}$ é igual ao número de pontos $\overline{\boldsymbol{x}}$ onde $\overline{\boldsymbol{u}}$ é definido, $\boldsymbol{P}$ é quadrada e normalmente pode ser invertida. Essa é a situação mais comum entre os elementos finitos, quando o número de nós é igual ao número de monômios da base de aproximação, e leva a

$$
\begin{aligned}
\boldsymbol{a}^{e} & =\boldsymbol{P}^{-1} \overline{\boldsymbol{u}} \\
u^{e}(\boldsymbol{x}) & =\boldsymbol{p}(\boldsymbol{x}) \cdot \boldsymbol{P}^{-1} \overline{\boldsymbol{u}}=\boldsymbol{\Phi}(\boldsymbol{x}) \overline{\boldsymbol{u}}
\end{aligned},
$$

sendo $\boldsymbol{\Phi}(\boldsymbol{x})=\boldsymbol{p}^{T} \boldsymbol{P}^{-1}$ o conjunto das funções de forma atreladas aos coeficientes $\overline{\boldsymbol{u}}$. É interessante notar que, nas formulações mais comuns de elementos finitos, a função de forma $\Phi_{i}$ obedece à propriedade de delta de Kronecker, ou seja,

$$
\Phi_{i}\left(\overline{\boldsymbol{x}}_{j}\right)=\delta_{i j}
$$

a função de forma assume valor unitário no nó ao qual está associada e zero em todos os outros nós, o que dá ao parâmetro nodal um significado físico: naquele ponto, a função a ser aproximada tem aquele valor

$$
u^{h}\left(\overline{\boldsymbol{x}}_{i}\right)=\bar{u}_{i}
$$

Esse processo também pode ser descrito como a minimização da norma do erro entre a aproximação e o parâmetro nodal ( $\left.J^{e}\right)$ nos nós. 


$$
\begin{aligned}
J^{e}\left(\boldsymbol{a}^{e}\right) & =\sum_{i=1}^{n^{e}}\left(\bar{u}_{i}-u^{e}\left(\boldsymbol{x}_{i}\right)\right)^{2}=\sum_{i=1}^{n^{e}}\left(\bar{u}_{i}-\boldsymbol{p}\left(\boldsymbol{x}_{i}\right) \cdot \boldsymbol{a}^{e}\right)^{2}, \\
& =\left(\overline{\boldsymbol{u}}-\boldsymbol{P} \boldsymbol{a}^{e}\right) \cdot\left(\overline{\boldsymbol{u}}-\boldsymbol{P} \boldsymbol{a}^{e}\right)^{2}
\end{aligned}
$$

onde $P_{i j}=p_{j}\left(\overline{\boldsymbol{x}}_{i}\right)$. A minimização de $J^{e}\left(\boldsymbol{a}^{e}\right)$ com relação a $\boldsymbol{a}$ leva a (2.4). Mantendo-se a igualdade de nós por elemento com a dimensão da base polinomial, esse erro é zero nos nós e (2.6) é obedecida.

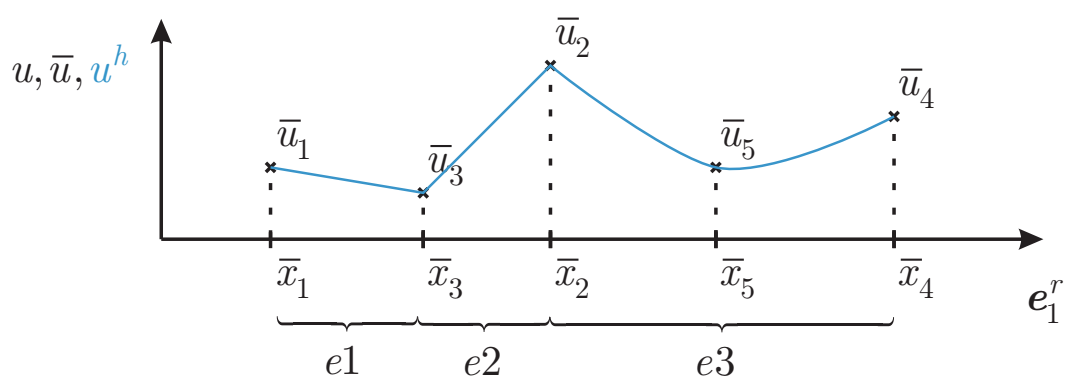

Figura 2 - Aproximação com Elementos Finitos

A Figura 2 mostra esquematicamente a aproximação obtida no caso anterior.

\subsection{Mínimos Quadrados}

O esquema de Mínimos Quadrados parte da minimização do erro da aproximação nos pontos onde o valor da função é definido. A ideia de se aproximar $u(\boldsymbol{x})$ através de uma combinação linear das funções de forma se mantem, mas a aproximação agora não depende da divisão do domínio em elementos. Cria-se uma distribuição de pontos $\overline{\boldsymbol{x}}$ (no domínio ou próximo a ele, seguindo condições descritas por Tiago [2]) aos quais estarão associados os parâmetros de aproximação $\overline{\boldsymbol{u}}$.

Possui um aspecto geral na forma

$$
u^{h}(\boldsymbol{x})=\boldsymbol{p}(\boldsymbol{x}) \cdot \boldsymbol{a},
$$

em que a definição dos coeficientes $\boldsymbol{a}$ varia conforme a modalidade de Least Squares a ser adotada. 
Sua forma clássica é descrita com $\boldsymbol{a}$ constante em todo o domínio. Exemplos de aproximações de diferentes ordens podem ser vistos na Figura 3.

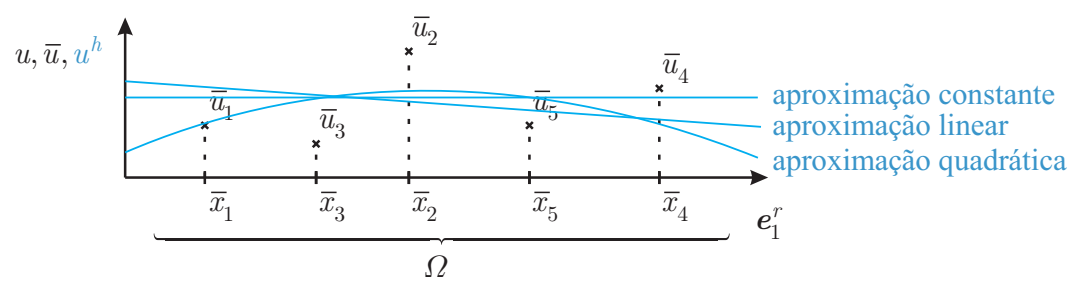

Figura 3 - Aproximações com Least Squares

A norma do erro da aproximação nas partículas será

$$
\begin{aligned}
J(\boldsymbol{a}) & =\sum_{i=1}^{n}\left(\bar{u}_{i}-u^{h}\left(\overline{\boldsymbol{x}}_{i}\right)\right)^{2} \\
& =\sum_{i=1}^{n}\left(\bar{u}_{i}-\boldsymbol{p}\left(\overline{\boldsymbol{x}}_{i}\right) \cdot \boldsymbol{a}\right)^{2} \\
& =(\overline{\boldsymbol{u}}-\boldsymbol{P a})^{T}(\overline{\boldsymbol{u}}-\boldsymbol{P a})
\end{aligned}
$$

Agora, pouco pode se afirmar sobre as dimensões de $\boldsymbol{P}$. Normalmente, têm-se mais partículas no domínio que membros na base de aproximação, o que torna $\boldsymbol{P}$ retangular. A minimização de (2.9) em relação aos parâmetros $\boldsymbol{a}$ leva a

$$
\frac{\partial J(\boldsymbol{a})}{\partial \boldsymbol{a}}=\boldsymbol{P}^{T} \boldsymbol{P a}-\boldsymbol{P}^{T} \overline{\boldsymbol{u}}=\boldsymbol{O}
$$

ou

$$
\begin{aligned}
& \boldsymbol{A} \boldsymbol{a}=\boldsymbol{B} \overline{\boldsymbol{u}}, \\
& \boldsymbol{A}=\boldsymbol{P}^{T} \boldsymbol{P}, \\
& \boldsymbol{B}=\boldsymbol{P}^{T}
\end{aligned}
$$

que pode ser resolvido para $\boldsymbol{a}$,

$$
a=A^{-1} B \bar{u}
$$


e as funções de forma serão

$$
\begin{aligned}
& \Phi_{i}(\boldsymbol{x})=\boldsymbol{p}(\boldsymbol{x}) \boldsymbol{A}^{-1} \boldsymbol{B}_{i}, \\
& \boldsymbol{\Phi}(\boldsymbol{x})=\boldsymbol{p}(\boldsymbol{x}) \boldsymbol{A}^{-1} \boldsymbol{B}
\end{aligned}
$$

onde $\boldsymbol{B}_{i}$ é a $i$-ésima coluna de $\boldsymbol{B}$.

A forma clássica dos mínimos quadrados carrega consigo algumas deficiências drásticas que dificultam sua aplicabilidade. A aproximação tem um caráter global, ou seja, todas as partículas contribuem de forma igual para a aproximação em todos os pontos. Pode-se recorrer a uma ponderação do erro a fim de concentrar a aproximação em uma determinada região, mas a mesma continuaria distante dos parâmetros nodais em pontos longe do centro de ponderação.

Também, um aumento no número de funções da base, normalmente um acréscimo de monômios de ordem superior, causa um mau condicionamento do sistema de equações devido às diferentes ordens de grandeza dos membros de $\boldsymbol{p}(\boldsymbol{x})$ quando avaliados longe da origem.

\subsection{Distance-Weighted Least Squares ou Moving Least Squares}

Há algumas maneiras de se contornar as dificuldades dos Mínimos Quadrados. O parâmetro $\boldsymbol{a}$ pode ser tomado variável no domínio. Assim, para cada ponto $\boldsymbol{x}$ o valor aproximação nos pontos $\overline{\boldsymbol{x}}$ será diferente. A ponderação do erro permite, então, que a aproximação nas partículas mais próximas tenda a $\overline{\boldsymbol{u}}$, enquanto progressivamente despreza o erro nas mais distantes.

A imposição desse peso ao erro provê um caráter local à aproximação e a escolha da função peso é de grande importância para a eficiência do método. Discussões sobre as características recomendáveis às mesmas podem ser encontradas na bibliografia (recomenda-se [2] e [16]). Esse texto limita-se a citar algumas: 
- isotropia: deve assumir uma forma isotrópica, sem favorecer uma direção sobre a outra, o que pode ser facilmente obtido tomando funções cujo argumento seja a distância entre pontos;

- positividade: a função deve ser positiva em todo seu domínio;

- suporte compacto: a função deve assumir o valor zero a partir de certa distância;

- normalidade: a integral da função em seu suporte deve ser 1;

- monotonamente decrescente: a função deve decair conforme aumenta a distância para o seu centro;

- comportamento de delta de Dirac: deve tender ao delta de Dirac enquanto seu domínio é reduzido.

Algumas funções peso devem ser citadas por serem usadas ou referenciadas nesse texto, o que não diminui a qualidade de outras opções. Seja $r$ a distância entre o centro da função peso e o ponto onde se deve avaliá-la,

constante:

$$
W(r)=c
$$

constante no intervalo: $\quad W(r)=\left\{\begin{array}{l}c, \text { se } r \leq 1 \\ 0, \text { se } r>1\end{array}\right.$

spline cúbico: $W(r)=\left\{\begin{array}{l}\frac{2}{3}-4 r^{2}+4 r^{3}, \text { se } r \leq \frac{1}{2} \\ \frac{4}{3}-4 r+4 r^{2}-\frac{4}{3} r^{3}, \text { se } \frac{1}{2}<r \leq 1 \\ 0, \text { se } r>1\end{array}\right.$

spline ajustável: $\quad W(r)=\left\{\begin{array}{l}\left(1-r^{2}\right)^{s}, \text { se } r \leq 1 \\ 0, \text { se } r>1\end{array}\right.$

onde $c$ é uma constante real e $s$ é um número inteiro positivo. 
A função spline ajustável com parâmetro $s$ igual a 3 foi a utilizada na maior parte dos exemplos numéricos, e encontra-se esquematizada em uma e duas dimensões na Figura 4.
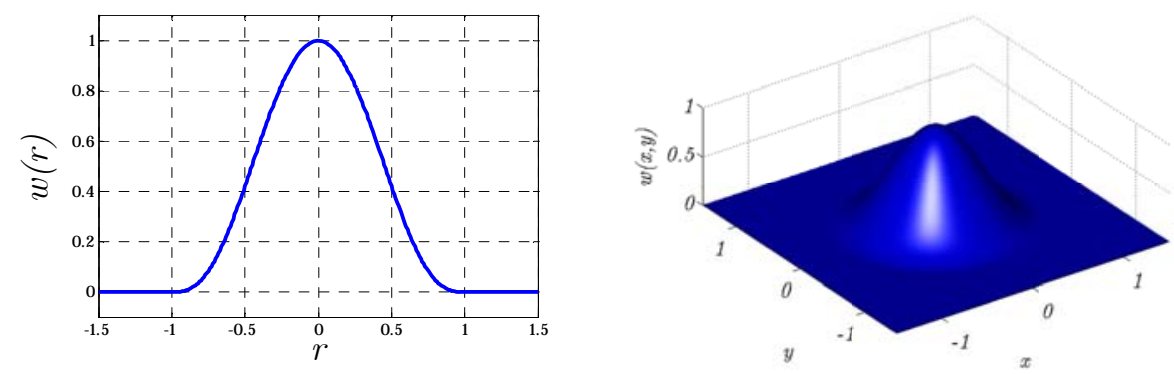

Figura 4 - Spline ajustável de grau 3 (uma e duas dimensões)

Definida a função peso, a aproximação em uma região em torno de um ponto $\tilde{\boldsymbol{x}}$ será dada por

$$
u^{\tilde{x}}(\boldsymbol{x})=\boldsymbol{p}(\boldsymbol{x}) \cdot \boldsymbol{a}(\tilde{\boldsymbol{x}})
$$

É interessante notar que a aproximação não depende apenas da posição do ponto de interesse, mas também do ponto de referência $\tilde{\boldsymbol{x}}$. Para as partículas $\overline{\boldsymbol{x}}$, esta aproximação será

$$
\begin{aligned}
& u_{i}^{\tilde{x}}=\boldsymbol{p}\left(\overline{\boldsymbol{x}}_{i}\right) \cdot \boldsymbol{a}(\tilde{\boldsymbol{x}}) \\
& \boldsymbol{u}^{\tilde{x}}=\boldsymbol{P a}(\tilde{\boldsymbol{x}})
\end{aligned}
$$

onde, mais uma vez, $P_{i j}=p_{j}\left(\overline{\boldsymbol{x}}_{i}\right)$. Simplificando a notação com $\boldsymbol{a}(\tilde{\boldsymbol{x}})=\boldsymbol{a}^{\tilde{\boldsymbol{x}}}$ e $W(\|\boldsymbol{x}-\tilde{\boldsymbol{x}}\|)=w^{\tilde{\boldsymbol{x}}}(\boldsymbol{x})$, a norma do erro ponderado será

$$
\begin{aligned}
J^{\tilde{x}}\left(\boldsymbol{a}^{\tilde{x}}\right) & =\sum_{i=1}^{n} w^{\tilde{x}}\left(\overline{\boldsymbol{x}}_{i}\right)\left(\bar{u}_{i}-u^{\tilde{x}}\left(\overline{\boldsymbol{x}}_{i}\right)\right)^{2} \\
& =\sum_{i=1}^{n} w^{\tilde{x}}\left(\overline{\boldsymbol{x}}_{i}\right)\left(\bar{u}_{i}-\boldsymbol{p}\left(\overline{\boldsymbol{x}}_{i}\right) \cdot \boldsymbol{a}(\tilde{\boldsymbol{x}})\right)^{2}, \\
& =\left(\overline{\boldsymbol{u}}-\boldsymbol{P} \boldsymbol{a}^{\tilde{x}}\right)^{T} \boldsymbol{W}\left(\overline{\boldsymbol{u}}-\boldsymbol{P} \boldsymbol{a}^{\tilde{x}}\right)
\end{aligned}
$$

onde $W_{i i}=w^{\tilde{x}}\left(\overline{\boldsymbol{x}}_{i}\right)$. A minimização de (2.20) em $\boldsymbol{a}^{\tilde{\boldsymbol{x}}}$ leva a

$$
\boldsymbol{A}^{\tilde{x}} \boldsymbol{a}^{\tilde{x}}=\boldsymbol{B}^{\tilde{x}} \bar{u} .
$$


O processo se torna móvel a partir da imposição que $\tilde{\boldsymbol{x}} \rightarrow \boldsymbol{x}$, ou seja, que o centro da aproximação, especificamente da função peso, é o próprio ponto de interesse. Dessa forma,

$$
\boldsymbol{a}(\boldsymbol{x})=\boldsymbol{A}^{-1}(\boldsymbol{x}) \boldsymbol{B}(\boldsymbol{x}) \overline{\boldsymbol{u}}
$$

sendo

$$
\begin{aligned}
\boldsymbol{A}^{\tilde{x}} & =\sum_{i=1}^{n} w^{\tilde{x}}\left(\overline{\boldsymbol{x}}_{i}\right) \boldsymbol{A}_{i} \\
\boldsymbol{A}_{i} & =\boldsymbol{p}\left(\overline{\boldsymbol{x}}_{i}\right) \boldsymbol{p}\left(\overline{\boldsymbol{x}}_{i}\right)^{T} \\
\boldsymbol{B}^{\tilde{x}} & =\left[\begin{array}{llll}
w^{\tilde{x}}\left(\overline{\boldsymbol{x}}_{1}\right) \boldsymbol{p}\left(\overline{\boldsymbol{x}}_{1}\right) & w^{\tilde{x}}\left(\overline{\boldsymbol{x}}_{2}\right) \boldsymbol{p}\left(\overline{\boldsymbol{x}}_{2}\right) & \cdots & w^{\tilde{x}}\left(\overline{\boldsymbol{x}}_{n}\right) \boldsymbol{p}\left(\overline{\boldsymbol{x}}_{n}\right)
\end{array}\right]
\end{aligned}
$$

Substituindo (2.22) em (2.18),

$$
\begin{aligned}
& u^{\tilde{x}}(\boldsymbol{x})=\boldsymbol{p}(\boldsymbol{x}) \boldsymbol{A}^{-1}(\boldsymbol{x}) \boldsymbol{B}(\boldsymbol{x}) \overline{\boldsymbol{u}} \\
& \Phi_{i}(\boldsymbol{x})=\boldsymbol{p}(\boldsymbol{x}) \boldsymbol{A}^{-1}(\boldsymbol{x}) \boldsymbol{B}_{i}(\boldsymbol{x})
\end{aligned}
$$

onde $\boldsymbol{B}_{i}$ é a $i$-ésima coluna de $\boldsymbol{B}$.

As derivadas da função de forma são

$$
\boldsymbol{\Phi}_{, i}(\boldsymbol{x})=\boldsymbol{p}_{, i} \boldsymbol{A}^{-1} \boldsymbol{B}+\boldsymbol{p}\left[\boldsymbol{A}^{-1}\right]_{, i} \boldsymbol{B}+\boldsymbol{p} \boldsymbol{A}^{-1} \boldsymbol{B}_{, i} .
$$

As derivadas da inversa da matriz $\boldsymbol{A}$ podem ser obtidas por

$$
\left[\boldsymbol{A}^{-1}\right]_{, i}=-\boldsymbol{A}^{-1} \boldsymbol{A}_{, i} \boldsymbol{A}^{-1}
$$

A Figura 5 esquematiza aproximações constantes, lineares e quadráticas em torno do ponto $\tilde{\boldsymbol{x}}$ em uma dimensão. 


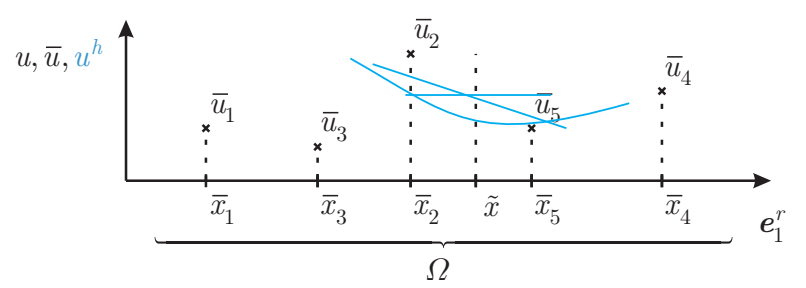

Figura 5 - Aproximações com Moving Least Squares

Alguns procedimentos são utilizados para melhorar a desempenho dessa aproximação. Uma observação é feita por Krys1 e Belytschko em [18], que salienta a importância de se substituir nessas aproximações as coordenadas de $\boldsymbol{x}$ por $\boldsymbol{x}-\tilde{\boldsymbol{x}}$ para evitar-se perda de precisão e mau condicionamento por conta de que os valores absolutos de $\boldsymbol{x}$ são demasiado grandes. Isso significa usar um sistema local de coordenadas, centrado no ponto de interesse, para a base da aproximação.

\subsection{Multiple Fixed Least Squares}

Os Mínimos Quadrados Móveis possuem uma definição clara e objetiva, mas sua implementação numérica é um tanto dificultosa. Particularmente, a definição de uma regra de variação da função peso sobre o domínio, necessária para o cômputo de $w^{\tilde{\boldsymbol{x}}}(\boldsymbol{x})$, é sujeita a um grande número de condições.

Assim, na maioria das implementações, a função peso está realmente definida nas partículas, ao que se chama de Mínimos Quadrados Fixos Múltiplos (Multiple Fixed Least Squares). Pode-se citar o caso de Dolbow e Belytschko [16], onde o texto define a aproximação por MLS enquanto o código fornecido no anexo usa os MFLS.

Nos Multiple Fixed Least Squares, a função peso é centrada nas partículas. Uma fácil interpretação da diferença é na definição das nuvens. Enquanto que nos Mínimos Quadrados Móveis vão-se buscar partículas que estejam no suporte de $w^{\tilde{x}}$, nos Mínimos Quadrados Fixos Múltiplos vão-se buscar as partículas em cujo domínio $\boldsymbol{x}$ se encontre. A função peso é definida para cada partícula, e não para cada ponto do domínio e naquelas que estão seus centros. 
Neste trabalho, toda a implementação computacional é feita em Multiple Fixed Least Squares, mesmo quando se fala em Moving Least Squares. De fato, a função peso é definida como igual para todo o domínio, sendo que os parâmetros que definem seu suporte são guardados para cada partícula. São determinados percorrendo-se os pontos de interesse do problema (pontos de amostragem na regra de integração, pontos de aplicação de forças, de restrições cinemáticas e de interesse no traçado de resultados) e inspecionando se o número de partículas cujo suporte inclui tal ponto é suficiente para que a base seja bem determinada ali (se $\boldsymbol{A}$ é invertível). Então, são majorados por um coeficiente $d_{\text {max }}$. 


\section{PLACAS SOB LINEARIDADE GEOMÉTRICA}

Pretende-se, neste capítulo, descrever as teorias de placas espessas e de elasticidade plana de forma unificada. Assim, será demonstrada uma cinemática em que os deslocamentos provenientes das duas condições são superpostos, dentro de uma abordagem geometricamente linear.

Dessa forma, definem-se as deformações de membrana e de flexão consistentemente, bem como suas respectivas tensões generalizadas. Servirá, assim, de base comparativa para a teoria linear de cascas apresentada a posteriori.

Em seguida, a forma fraca resultante é discretizada em Moving Least Squares e são mostrados os resultados obtidos em exemplos numéricos.

\subsection{Cinemática}

Seja uma placa plana colocada no plano de $\left\{\boldsymbol{e}_{1}^{r}, e_{2}^{r}\right\}$. A posição dos pontos da placa pode ser descrita como $\boldsymbol{\xi}=\boldsymbol{\zeta}+\boldsymbol{a}^{r}$, onde $\boldsymbol{\zeta}=\xi_{\alpha} \boldsymbol{e}_{\alpha}^{r}$ localiza o ponto na superfície de referência e $\boldsymbol{a}^{r}=\xi_{3} \boldsymbol{e}_{3}^{r}$ é o diretor, perpendicular à mesma.

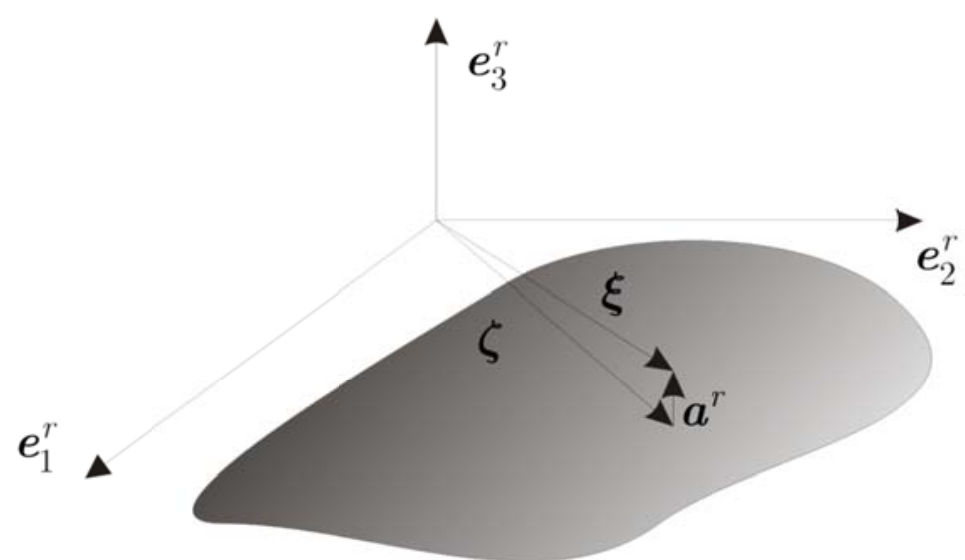

Figura 6 - Placa plana

Os deslocamentos na Teoria de Reissner-Mindlin são dados por 


$$
\boldsymbol{\delta}=\boldsymbol{u}+\boldsymbol{\theta} \times \boldsymbol{a}^{r}
$$

onde $\boldsymbol{u}=\boldsymbol{u}\left(\xi_{\alpha}\right)$ é o vetor deslocamento da superfície média e $\boldsymbol{\theta}=\boldsymbol{\theta}\left(\xi_{\alpha}\right)$ é o vetor rotação, definido no plano da superfície média, ou seja,

$$
\boldsymbol{\theta}=\theta_{\alpha} e_{\alpha}
$$

Vale ressaltar que $u$ possui 3 componentes, já que pretende-se descrever simultaneamente os movimentos de chapa $\left(\boldsymbol{u}_{\alpha}\right)$ e de placa $\left(\boldsymbol{u}_{3}\right.$ e $\left.\boldsymbol{\theta}_{\alpha}\right)$.

O gradiente dos deslocamentos é dado por

$$
\begin{aligned}
\boldsymbol{L} & =\frac{\partial \boldsymbol{\delta}}{\partial \boldsymbol{\xi}}=\frac{\partial \boldsymbol{\delta}}{\partial \xi_{\alpha}} \otimes \boldsymbol{e}_{\alpha}^{r}+\frac{\partial \boldsymbol{\delta}}{\partial \xi_{3}} \otimes \boldsymbol{e}_{3}^{r} \\
& =\left(\boldsymbol{u}_{, \alpha}+\boldsymbol{\theta}_{, \alpha} \times \boldsymbol{a}^{r}\right) \otimes \boldsymbol{e}_{\alpha}^{r}+\left(\boldsymbol{\theta} \times \boldsymbol{a}_{, 3}^{r}\right) \otimes \boldsymbol{e}_{3}^{r} \\
& =\left(\boldsymbol{u}_{, \alpha}-\boldsymbol{\theta} \times \boldsymbol{e}_{\alpha}^{r}+\boldsymbol{\theta}_{, \alpha} \times \boldsymbol{a}^{r}\right) \otimes \boldsymbol{e}_{\alpha}^{r}+\left(\boldsymbol{\theta} \times \boldsymbol{e}_{\alpha}^{r}\right) \otimes \boldsymbol{e}_{\alpha}^{r}+\left(\boldsymbol{\theta} \times \boldsymbol{e}_{3}^{r}\right) \otimes \boldsymbol{e}_{3}^{r} . \\
& =\left(\boldsymbol{u}_{, \alpha}+\boldsymbol{e}_{\alpha}^{r} \times \boldsymbol{\theta}+\boldsymbol{\theta}_{, \alpha} \times \boldsymbol{a}^{r}\right) \otimes \boldsymbol{e}_{\alpha}^{r}+\left(\boldsymbol{\theta} \times \boldsymbol{e}_{i}^{r}\right) \otimes \boldsymbol{e}_{i}^{r} \\
& =\left(\boldsymbol{\eta}_{\alpha}+\boldsymbol{\kappa}_{a} \times \boldsymbol{a}^{r}\right) \otimes \boldsymbol{e}_{\alpha}^{r}+\boldsymbol{\Theta} \\
& =\boldsymbol{\gamma}_{\alpha} \otimes \boldsymbol{e}_{\alpha}^{r}+\boldsymbol{\Theta}
\end{aligned}
$$

com a definição das deformações generalizadas de membrana e de flexão, respectivamente

$$
\boldsymbol{\eta}_{\alpha}=\boldsymbol{u}_{, \alpha}+\boldsymbol{e}_{\alpha}^{r} \times \boldsymbol{\theta} \text { e } \quad \boldsymbol{\kappa}_{\alpha}=\boldsymbol{\theta}_{, \alpha}
$$

e o tensor antissimétrico cujo vetor axial é $\boldsymbol{\theta}$

$$
\boldsymbol{\Theta}=\operatorname{Skew}(\boldsymbol{\theta})
$$

sendo as deformações generalizadas em um ponto dadas por

$$
\gamma_{\alpha}=\boldsymbol{\eta}_{\alpha}+\boldsymbol{\kappa}_{\alpha} \times \boldsymbol{a}^{r}
$$


Nota-se que

$$
\begin{aligned}
\boldsymbol{e}_{3}^{r} \cdot \boldsymbol{\eta}_{\alpha} & =\boldsymbol{e}_{3}^{r} \cdot\left(\boldsymbol{u}_{, \alpha}+\boldsymbol{e}_{\alpha}^{r} \times \boldsymbol{\theta}\right)=\boldsymbol{e}_{3}^{r} \cdot \boldsymbol{u}_{, \alpha}+\boldsymbol{e}_{3}^{r} \cdot\left(\boldsymbol{e}_{\alpha}^{r} \times \boldsymbol{\theta}\right) \\
\boldsymbol{e}_{3}^{r} \cdot \boldsymbol{\eta}_{\alpha} & =\boldsymbol{e}_{3}^{r} \cdot \boldsymbol{u}_{, \alpha}+\left(\boldsymbol{e}_{3}^{r} \times \boldsymbol{e}_{\alpha}^{r}\right) \cdot \boldsymbol{\theta} \\
\left(\boldsymbol{e}_{\alpha}^{r} \times \boldsymbol{e}_{3}^{r}\right) \cdot \boldsymbol{\theta} & =\boldsymbol{e}_{3}^{r} \cdot\left(\boldsymbol{u}_{, \alpha}-\boldsymbol{\eta}_{\alpha}\right) \\
-\varepsilon_{\alpha \beta} \boldsymbol{e}_{\beta}^{r} \cdot \boldsymbol{\theta} & =\boldsymbol{e}_{3}^{r} \cdot\left(\boldsymbol{u}_{, \alpha}-\boldsymbol{\eta}_{\alpha}\right) \\
\boldsymbol{e}_{\beta}^{r} \cdot \boldsymbol{\theta} & =-\varepsilon_{\alpha \beta} \boldsymbol{e}_{3}^{r} \cdot\left(\boldsymbol{u}_{, \alpha}-\boldsymbol{\eta}_{\alpha}\right) \\
\boldsymbol{e}_{\alpha}^{r} \cdot \boldsymbol{\theta} & =\varepsilon_{\alpha \beta} \boldsymbol{e}_{3}^{r} \cdot\left(\boldsymbol{u}_{, \beta}-\boldsymbol{\eta}_{\beta}\right)
\end{aligned}
$$

onde

$$
\varepsilon_{\alpha \beta}=\boldsymbol{e}_{3}^{r} \cdot \boldsymbol{e}_{\alpha}^{r} \times \boldsymbol{e}_{\beta}^{r}
$$

Se o desejo fosse de uma teoria de placas finas, poder-se-ia expressar o vínculo de Kirchhoff pela ausência do cisalhamento, a terceira componente dos vetores de deformação generalizada, usando (3.7):

$$
\begin{aligned}
& \boldsymbol{e}_{3}^{r} \cdot \boldsymbol{\eta}_{\alpha}=0 \\
& \boldsymbol{e}_{\alpha}^{r} \cdot \boldsymbol{\theta}=\varepsilon_{\alpha \beta} \boldsymbol{e}_{3}^{r} \cdot \boldsymbol{u}_{, \beta}
\end{aligned}
$$

ou, em sua forma clássica, por

$$
\begin{aligned}
& \theta_{1}=u_{3,2} \\
& \theta_{2}=-u_{3,1}
\end{aligned} .
$$

\subsection{Estática}

O tensor de Cauchy pode ser representado por seus vetores-coluna $t_{i}$ como em

$$
\boldsymbol{T}=\boldsymbol{t}_{i} \otimes \boldsymbol{e}_{i}^{r}
$$

Sua simetria pode ser expressa por 


$$
\begin{aligned}
& \boldsymbol{e}_{i}^{r} \times \boldsymbol{t}_{i}=\boldsymbol{O} \\
& \boldsymbol{e}_{\alpha}^{r} \times \boldsymbol{t}_{\alpha}+\boldsymbol{e}_{3}^{r} \times \boldsymbol{t}_{3}=\boldsymbol{O}
\end{aligned}
$$

de forma que

$$
\begin{aligned}
\boldsymbol{t}_{3} \cdot \boldsymbol{\delta}_{, 3} & =\boldsymbol{t}_{3} \cdot\left(\boldsymbol{\theta} \times \boldsymbol{e}_{3}\right) \\
& =\left(\boldsymbol{e}_{3} \times \boldsymbol{t}_{3}\right) \cdot \boldsymbol{\theta} \\
& =-\left(\boldsymbol{e}_{\alpha} \times \boldsymbol{t}_{\alpha}\right) \cdot \boldsymbol{\theta} \\
& =\boldsymbol{t}_{\alpha} \cdot\left(\boldsymbol{e}_{\alpha} \times \boldsymbol{\theta}\right)
\end{aligned}
$$

Assim, pode se desenvolver o produto

$$
\begin{aligned}
\boldsymbol{t}_{i} \cdot \boldsymbol{\delta}_{, i} & =\boldsymbol{t}_{\alpha} \cdot \boldsymbol{\delta}_{, \alpha}+\boldsymbol{t}_{3} \cdot \boldsymbol{\delta}_{, 3} \\
& =\boldsymbol{t}_{\alpha} \cdot \boldsymbol{u}_{, \alpha}+\boldsymbol{t}_{\alpha} \cdot\left(\boldsymbol{e}_{\alpha}^{r} \times \boldsymbol{\theta}\right) \\
& =\boldsymbol{t}_{\alpha} \cdot\left(\boldsymbol{u}_{, \alpha}+\boldsymbol{e}_{\alpha}^{r} \times \boldsymbol{\theta}\right) \\
& =\boldsymbol{t}_{\alpha} \cdot \boldsymbol{\gamma}_{\alpha}
\end{aligned}
$$

com $\gamma_{\alpha}$ definido em (3.6). Outra forma de se expressar a simetria de $\boldsymbol{T}$ é dada por

$$
\begin{aligned}
& \boldsymbol{t}_{(j)} \cdot \boldsymbol{e}_{(i)}^{r}=\boldsymbol{t}_{(i)} \cdot \boldsymbol{e}_{(j)}^{r} \\
& \boldsymbol{t}_{(\alpha)} \cdot \boldsymbol{e}_{(\beta)}^{r}=\boldsymbol{t}_{(\beta)} \cdot \boldsymbol{e}_{(\alpha)}^{r}, \\
& \boldsymbol{t}_{3} \cdot \boldsymbol{e}_{(\alpha)}^{r}=\boldsymbol{t}_{(\alpha)} \cdot \boldsymbol{e}_{3}^{r}
\end{aligned}
$$

onde os parênteses nos índices indicam que não há somatória.

A condição de estado plano de tensões é dada pela ausência de tensões nas faces cuja normal seja $e_{3}^{r}$

$$
t_{3} \cdot e_{3}=0
$$




\subsubsection{Esforços Generalizados}

Podemos definir os esforços generalizados como

$$
\boldsymbol{n}_{\alpha}=\int_{H} \boldsymbol{t}_{\alpha} d H \quad \text { e } \quad \boldsymbol{m}_{\alpha}=\int_{H} \boldsymbol{a}^{r} \times \boldsymbol{t}_{\alpha} d H
$$

onde $\boldsymbol{n}_{\alpha}$ reúne as resultantes de normal e cisalhamento e $\boldsymbol{m}_{\alpha}$ os momentos.

Da segunda expressão de (3.17), conclui-se que

$$
\begin{aligned}
\boldsymbol{m}_{\alpha} \cdot \boldsymbol{e}_{3}^{r} & =\int_{H}\left(\boldsymbol{a}^{r} \times \boldsymbol{t}_{\alpha}\right) d H \cdot \boldsymbol{e}_{3}^{r} \\
& =\int_{H}\left(\boldsymbol{e}_{3}^{r} \times \boldsymbol{t}_{\alpha}\right) \xi_{3} d H \cdot \boldsymbol{e}_{3}^{r} \\
& =\int_{H} \xi_{3} d H\left(\boldsymbol{e}_{3}^{r} \times \boldsymbol{t}_{\alpha}\right) \cdot \boldsymbol{e}_{3}^{r}=0
\end{aligned}
$$

o que indica que as tensões generalizadas de momento só terão componentes na direção do plano de referência devido à imposição do estado plano de tensões.

Os esforços $\boldsymbol{n}_{\alpha}$ podem ser decompostos da seguinte forma

$$
\boldsymbol{n}_{\alpha}=\boldsymbol{n}_{\alpha}^{m}+\boldsymbol{n}_{\alpha}^{b}
$$

onde

$$
\boldsymbol{n}_{\alpha}^{m}=\left(\boldsymbol{n}_{\alpha} \cdot \boldsymbol{e}_{\beta}^{r}\right) \boldsymbol{e}_{\beta}^{r}=N_{\alpha \beta} \boldsymbol{e}_{\beta}^{r} \quad \text { e } \quad \boldsymbol{n}_{\alpha}^{b}=\left(\boldsymbol{n}_{\alpha} \cdot \boldsymbol{e}_{3}^{r}\right) \boldsymbol{e}_{3}^{r}=V_{\alpha} \boldsymbol{e}_{3}^{r}
$$

são as parcelas de membrana e de placa, respectivamente. Com a ajuda de (3.15) e da primeira expressão de (3.17) chega-se a 


$$
\begin{aligned}
\boldsymbol{n}_{\alpha} \cdot \boldsymbol{e}_{\beta}^{r} & =\int_{H} \boldsymbol{t}_{\alpha} d H \cdot \boldsymbol{e}_{\beta}^{r} \\
& =\int_{H} \boldsymbol{t}_{\alpha} \cdot \boldsymbol{e}_{\beta}^{r} d H \\
& =\int_{H} \boldsymbol{t}_{\beta} \cdot \boldsymbol{e}_{\alpha}^{r} d H \\
& =\boldsymbol{n}_{\beta} \cdot \boldsymbol{e}_{\alpha}^{r}
\end{aligned}
$$

ou $N_{\alpha \beta}=N_{\beta \alpha}$. Com (3.15), tem-se

$$
\begin{aligned}
\int_{H} \boldsymbol{t}_{3} d H & =\int_{H}\left(\boldsymbol{t}_{3} \cdot \boldsymbol{e}_{\alpha}^{r}\right) \boldsymbol{e}_{\alpha}^{r} d H \\
& =\left(\int_{H}\left(\boldsymbol{t}_{\alpha} \cdot \boldsymbol{e}_{3}^{r}\right) d H\right) \boldsymbol{e}_{\alpha}^{r} \\
& =\left(\int_{H}\left(\boldsymbol{t}_{\alpha}\right) d H \cdot \boldsymbol{e}_{3}^{r}\right) \boldsymbol{e}_{\alpha}^{r} \\
& =\left(\boldsymbol{n}_{\alpha} \cdot \boldsymbol{e}_{3}^{r}\right) \boldsymbol{e}_{\alpha}^{r} \\
& =V_{\alpha} \boldsymbol{e}_{\alpha}^{r}
\end{aligned}
$$

Podem-se definir os seguintes tensores

$$
\boldsymbol{N}=\boldsymbol{n}_{\alpha} \otimes \boldsymbol{e}_{\alpha}^{r} \quad \text { e } \quad \boldsymbol{M}=\boldsymbol{m}_{\alpha} \otimes \boldsymbol{e}_{\alpha}^{r}
$$

Logo $N$ não é simétrico, mas

$$
\boldsymbol{N}^{m}=\boldsymbol{n}_{\alpha}^{m} \otimes \boldsymbol{e}_{\alpha}^{r}=N_{\alpha \beta} \boldsymbol{e}_{\alpha}^{r} \otimes \boldsymbol{e}_{\beta}^{r}
$$

é simétrico. Com o auxílio de (3.15), conclui-se também que

$$
\boldsymbol{m}_{2} \cdot \boldsymbol{e}_{2}^{r}=-\boldsymbol{m}_{1} \cdot \boldsymbol{e}_{1}^{r}
$$

note-se, no entanto, que $M$ não é simétrico.

\subsubsection{Potência Interna}

A potência dos esforços internos por unidade de volume da placa pode ser dada por 


$$
\begin{aligned}
\boldsymbol{T}: \dot{\boldsymbol{E}} & =\boldsymbol{T}: \frac{1}{2}\left[\frac{\cdot}{\left(\gamma_{\alpha} \otimes \boldsymbol{e}_{\alpha}^{r}+\boldsymbol{\Theta}\right)}+\overline{\left(\gamma_{\alpha} \otimes \boldsymbol{e}_{\alpha}^{r}+\boldsymbol{\Theta}\right)^{T}}\right] \\
& =\boldsymbol{T}: \frac{1}{2}\left[\dot{\gamma}_{\alpha} \otimes \boldsymbol{e}_{\alpha}^{r}+\dot{\boldsymbol{\Theta}}+\overline{\left(\boldsymbol{e}_{\alpha}^{r} \otimes \gamma_{\alpha}+\boldsymbol{\Theta}^{T}\right)}\right] \\
& =\boldsymbol{T}: \frac{1}{2}\left[\dot{\gamma}_{\alpha} \otimes \boldsymbol{e}_{\alpha}^{r}+\boldsymbol{e}_{\alpha}^{r} \otimes \dot{\gamma}_{\alpha}\right] \\
& =\boldsymbol{T}: \frac{1}{2}\left[\dot{\gamma}_{\alpha} \otimes \boldsymbol{e}_{\alpha}^{r}\right]+\boldsymbol{T}: \frac{1}{2}\left[\boldsymbol{e}_{\alpha}^{r} \otimes \dot{\gamma}_{\alpha}\right] \\
& =\left[\boldsymbol{t}_{i} \otimes \boldsymbol{e}_{i}^{r}\right]:\left[\dot{\gamma}_{\alpha} \otimes \boldsymbol{e}_{\alpha}^{r}\right] \\
& =\boldsymbol{t}_{\alpha} \cdot \dot{\gamma}_{\alpha}
\end{aligned}
$$

que, integrada na espessura, nos fornece a potência por unidade de área:

$$
\begin{aligned}
\int_{H}(\boldsymbol{T}: \dot{\boldsymbol{E}}) d H & =\int_{H}\left(\boldsymbol{t}_{\alpha} \cdot \dot{\boldsymbol{\gamma}}_{\alpha}\right) d H \\
& =\int_{H}\left[\boldsymbol{t}_{\alpha} \cdot \dot{\boldsymbol{\eta}}_{\alpha}+\boldsymbol{t}_{\alpha} \cdot\left(\dot{\boldsymbol{\kappa}}_{\alpha} \times \boldsymbol{a}^{r}\right)\right] d H \\
& =\int_{H}\left[\boldsymbol{t}_{\alpha} \cdot \dot{\boldsymbol{\eta}}_{\alpha}\right] d H+\int_{H}\left[\left(\boldsymbol{a}^{r} \times \boldsymbol{t}_{\alpha}\right) \cdot \dot{\boldsymbol{\kappa}}_{\alpha}\right] d H \\
& =\left[\int_{H}\left(\boldsymbol{t}_{\alpha}\right) d H\right] \cdot \dot{\boldsymbol{\eta}}_{\alpha}+\left[\int_{H}\left(\boldsymbol{a}^{r} \times \boldsymbol{t}_{\alpha}\right) d H\right] \cdot \dot{\boldsymbol{\kappa}}_{\alpha} \\
& =\boldsymbol{n}_{\alpha}^{r} \cdot \dot{\boldsymbol{\eta}}_{\alpha}+\boldsymbol{m}_{\alpha}^{r} \cdot \dot{\boldsymbol{\kappa}}_{\alpha}
\end{aligned}
$$

Podem-se ainda definir os vetores que reúnem as tensões, as deformações e os deslocamentos generalizados

$$
\boldsymbol{\sigma}_{\alpha}=\left[\begin{array}{c}
\boldsymbol{n}_{\alpha} \\
\boldsymbol{m}_{\alpha}
\end{array}\right], \quad \boldsymbol{\varepsilon}_{\alpha}=\left[\begin{array}{c}
\boldsymbol{\eta}_{\alpha} \\
\boldsymbol{\kappa}_{\alpha}
\end{array}\right] \quad \text { e } \boldsymbol{d}=\left[\begin{array}{c}
\boldsymbol{u} \\
\boldsymbol{\theta}
\end{array}\right]
$$

tal que

$$
\begin{aligned}
& \varepsilon_{\alpha}=\Delta_{\alpha} \boldsymbol{d} \\
& \dot{\varepsilon}_{\alpha}=\Delta_{\alpha} \dot{d}
\end{aligned}
$$

onde 


$$
\boldsymbol{\Delta}_{\alpha}=\left[\begin{array}{cc}
\boldsymbol{I} \frac{\partial}{\partial \xi_{\alpha}} & \boldsymbol{E}_{\alpha}^{r} \\
\boldsymbol{O} & \boldsymbol{I} \frac{\partial}{\partial \xi_{\alpha}}
\end{array}\right], \quad \boldsymbol{E}_{\alpha}^{r}=\operatorname{Skew}\left(\boldsymbol{e}_{\alpha}^{r}\right)
$$

Assim, (3.27) pode ser expressa por

$$
\begin{aligned}
\int_{H}(\boldsymbol{T}: \dot{\boldsymbol{E}}) d H & =\boldsymbol{n}_{\alpha}^{r} \cdot \dot{\boldsymbol{\eta}}_{\alpha}+\boldsymbol{m}_{\alpha}^{r} \cdot \dot{\boldsymbol{\kappa}}_{\alpha} \\
& =\boldsymbol{\sigma}_{\alpha}^{r} \cdot \dot{\boldsymbol{\varepsilon}}_{\alpha}^{r}
\end{aligned}
$$

\subsubsection{Potência Externa}

Definindo-se os vetores

$$
\boldsymbol{q}^{\Gamma_{u}}=\left[\begin{array}{c}
\boldsymbol{n}^{\Gamma} \\
\boldsymbol{m}^{\Gamma}
\end{array}\right]
$$

que reúne as reações na fronteira cinemática,

$$
\overline{\boldsymbol{q}}^{\Omega}=\left[\begin{array}{c}
\overline{\boldsymbol{n}}^{\Omega} \\
\overline{\boldsymbol{m}}^{\Omega}
\end{array}\right] \quad \text { e } \quad \overline{\boldsymbol{q}}^{\Gamma_{t}}=\left[\begin{array}{c}
\overline{\boldsymbol{n}}^{\Gamma_{t}} \\
\overline{\boldsymbol{m}}^{\Gamma_{t}}
\end{array}\right]
$$

que reúnem, respectivamente, os esforços generalizados impostos no domínio e na fronteira, a potência dos esforços externos será dada por

$$
P_{\mathrm{ext}}=\int_{\Omega}\left(\overline{\boldsymbol{q}}^{\Omega} \cdot \dot{\boldsymbol{d}}\right) d \Omega+\int_{\Gamma_{t}}\left(\overline{\boldsymbol{q}}^{\Gamma_{t}} \cdot \dot{\boldsymbol{d}}\right) d \Gamma_{t}+\int_{\Gamma_{u}}\left(\boldsymbol{q}^{\Gamma_{u}} \cdot \dot{\boldsymbol{d}}\right) d \Gamma_{u} .
$$

É interessante notar a presença das reações de apoio na definição da potência dos esforços externos. Isso se faz necessário uma vez que o tipo de aproximação escolhida para o desenvolvimento deste trabalho não impõe a priori as condições essenciais de contorno. 


\subsection{Forma Fraca}

A variação do vetor das deformações generalizadas dado por (3.6) pode ser feita na forma

$$
\delta \boldsymbol{\gamma}_{\alpha}=\delta \boldsymbol{\eta}_{\alpha}+\delta \boldsymbol{\kappa}_{\alpha} \times \boldsymbol{a}^{r}
$$

com

$$
\begin{aligned}
& \delta \boldsymbol{\eta}_{\alpha}=\delta \boldsymbol{w}_{, \alpha}+\boldsymbol{e}_{\alpha}^{r} \times \delta \boldsymbol{\theta} \\
& \delta \boldsymbol{\kappa}_{\alpha}=\delta \boldsymbol{\theta}_{, \alpha}
\end{aligned}
$$

ou seja, a variação das deformações generalizadas é análoga a (3.29),

$$
\delta \varepsilon_{\alpha}=\Delta_{\alpha} \delta \boldsymbol{d}
$$

Então, os trabalhos virtuais podem ser escritos como

$$
\delta W_{\mathrm{int}}=\int_{\Omega}\left(\boldsymbol{\sigma}_{\alpha} \cdot \delta \boldsymbol{\varepsilon}_{\alpha}\right) d \Omega
$$

e

$$
\delta W_{\mathrm{ext}}=\int_{\Omega}\left(\overline{\boldsymbol{q}}^{\Omega} \cdot \delta \boldsymbol{d}\right) d \Omega+\int_{\Gamma_{t}}\left(\overline{\boldsymbol{q}}^{\Gamma_{t}} \cdot \delta \boldsymbol{d}\right) d \Gamma_{t}+\int_{\Gamma_{u}}\left(\boldsymbol{q}^{\Gamma_{u}} \cdot \delta \boldsymbol{d}\right) d \Gamma_{u}
$$

Onde mais uma vez aparece a contribuição das reações de apoio, conforme já descrito na seção anterior.

Pelo mesmo motivo, a imposição das condições essenciais de contorno será feita pela imposição fraca da compatibilidade, expressa pelo trabalho virtual complementar das reações de apoio: 


$$
-\int_{\Gamma_{u}} \delta \boldsymbol{q}^{\Gamma_{u}} \cdot(\boldsymbol{d}-\overline{\boldsymbol{d}})=0, \forall \delta \boldsymbol{q}^{\Gamma_{u}}
$$

A combinação do Princípio dos Trabalhos Virtuais com essa imposição nos dá a forma fraca final, que será o funcional híbrido

$$
\begin{aligned}
\delta W= & \int_{\Omega}\left(\boldsymbol{\sigma}_{\alpha} \cdot \delta \boldsymbol{\varepsilon}_{\alpha}\right) d \Omega-\int_{\Omega}\left(\overline{\boldsymbol{q}}^{\Omega} \cdot \delta \boldsymbol{d}\right) d \Omega-\int_{\Gamma_{t}}\left(\overline{\boldsymbol{q}}^{\Gamma_{t}} \cdot \delta \boldsymbol{d}\right) d \Gamma_{t} \\
& -\int_{\Gamma_{u}}\left(\boldsymbol{q}^{\Gamma_{u}} \cdot \delta \boldsymbol{d}\right) d \Gamma_{u}-\int_{\Gamma_{u}}\left(\delta \boldsymbol{q}^{\Gamma_{u}} \cdot(\boldsymbol{d}-\overline{\boldsymbol{d}})\right) d \Gamma_{u}=0, \forall \delta \boldsymbol{d}, \delta \boldsymbol{q}^{\Gamma_{u}} .
\end{aligned}
$$

\subsection{Recuperação das equações de equilíbrio}

As equações locais de equilíbrio podem ser obtidas da forma fraca derivada anteriormente. Tomando o primeiro termo de (3.41) e integrando por partes em $\boldsymbol{u}_{, \alpha}$ e $\boldsymbol{\theta}_{, \alpha}$,

$$
\begin{aligned}
\int_{\Omega}\left(\boldsymbol{n}_{\alpha} \cdot \delta \boldsymbol{\eta}_{\alpha}+\boldsymbol{m}_{\alpha} \cdot \delta \boldsymbol{\kappa}_{\alpha}\right) d \Omega \\
=\int_{\Omega}\left(\boldsymbol{n}_{\alpha} \cdot\left(\delta \boldsymbol{u}_{, \alpha}+\boldsymbol{e}_{\alpha}^{r} \times \delta \boldsymbol{\theta}\right)+\boldsymbol{m}_{\alpha} \cdot \delta \boldsymbol{\theta}_{, \alpha}\right) d \Omega \\
=\int_{\Omega}\left(\boldsymbol{n}_{\alpha} \cdot \delta \boldsymbol{u}_{, \alpha}+\boldsymbol{n}_{\alpha} \cdot\left(\boldsymbol{e}_{\alpha}^{r} \times \delta \boldsymbol{\theta}\right)+\boldsymbol{m}_{\alpha} \cdot \delta \boldsymbol{\theta}_{, \alpha}\right) d \Omega \\
=\int_{\Omega}\left(\boldsymbol{n}_{\alpha} \cdot \delta \boldsymbol{u}_{, \alpha}\right) d \Omega+\int_{\Omega}\left(\boldsymbol{m}_{\alpha} \cdot \delta \boldsymbol{\theta}_{, \alpha}\right) d \Omega+\int_{\Omega}\left(\left(\boldsymbol{n}_{\alpha} \times \boldsymbol{e}_{\alpha}^{r}\right) \cdot \delta \boldsymbol{\theta}\right) d \Omega . \\
=\int_{\Gamma^{t}} n_{\alpha}\left(\boldsymbol{n}_{\alpha} \cdot \delta \boldsymbol{u}\right) d \Gamma^{t}+\int_{\Gamma^{u}} n_{\alpha}\left(\boldsymbol{n}_{\alpha} \cdot \delta \boldsymbol{u}\right) d \Gamma^{u}-\int_{\Omega}\left(\boldsymbol{n}_{\alpha, \alpha} \cdot \delta \boldsymbol{u}\right) d \Omega \\
\quad+\int_{\Gamma^{t}} n_{\alpha}\left(\boldsymbol{m}_{\alpha} \cdot \delta \boldsymbol{\theta}\right) d \Gamma^{t}+\int_{\Gamma^{u}} n_{\alpha}\left(\boldsymbol{m}_{\alpha} \cdot \delta \boldsymbol{\theta}\right) d \Gamma^{u}-\int_{\Omega}\left(\boldsymbol{m}_{\alpha, \alpha} \cdot \delta \boldsymbol{\theta}\right) d \Omega \\
\quad+\int_{\Omega}\left(\left(\boldsymbol{n}_{\alpha} \times \boldsymbol{e}_{\alpha}^{r}\right) \cdot \delta \boldsymbol{\theta}\right) d \Omega
\end{aligned}
$$

de forma que (3.41) pode ser escrita como 


$$
\begin{aligned}
& \delta W=\int_{\Gamma^{t}}\left(n_{\alpha} \boldsymbol{n}_{\alpha}\right) \cdot \delta \boldsymbol{u} d \Gamma^{t}+\int_{\Gamma^{u}}\left(n_{\alpha} \boldsymbol{n}_{\alpha}\right) \cdot \delta \boldsymbol{u} d \Gamma^{u}-\int_{\Omega}\left(\boldsymbol{n}_{\alpha, \alpha}\right) \cdot \delta \boldsymbol{u} d \Omega \\
& +\int_{\Gamma^{t}}\left(n_{\alpha} \boldsymbol{m}_{\alpha}\right) \cdot \delta \boldsymbol{\theta} d \Gamma^{t}+\int_{\Gamma^{u}}\left(n_{\alpha} \boldsymbol{m}_{\alpha}\right) \cdot \delta \boldsymbol{\theta} d \Gamma^{u}-\int_{\Omega}\left(\boldsymbol{m}_{\alpha, \alpha}\right) \cdot \delta \boldsymbol{\theta} d \Omega \\
& -\int_{\Omega}^{\Gamma^{t}}\left(\boldsymbol{e}_{\alpha}^{r} \times \boldsymbol{n}_{\alpha}\right) \cdot \delta \boldsymbol{\theta} d \Omega-\int_{\Omega}^{\Gamma^{u}}\left(\overline{\boldsymbol{n}}^{\Omega}\right) \cdot \delta \boldsymbol{u} d \Omega-\int_{\Omega}\left(\overline{\boldsymbol{m}}^{\Omega}\right) \cdot \delta \boldsymbol{\theta} d \Omega \\
& -\int_{\Gamma^{t}}^{\Omega}\left(\overline{\boldsymbol{n}}^{\Gamma_{t}}\right) \cdot \delta \boldsymbol{u} d \Gamma^{t}-\int_{\Gamma^{t}} \overline{\boldsymbol{m}}^{\Gamma_{t}} \cdot \delta \boldsymbol{\theta} d \Gamma^{t}-\int \stackrel{\Omega}{\boldsymbol{n}^{\Gamma_{u}}} \cdot \delta \boldsymbol{u} d \Gamma^{u} \\
& -\int_{\Gamma^{u}}^{\Gamma^{t}} \boldsymbol{m}^{\Gamma_{u}} \cdot \delta \boldsymbol{\theta} d \Gamma^{u}-\int_{\Gamma_{u}}^{\Gamma^{t}}\left(\delta \boldsymbol{q}^{\Gamma_{u}} \cdot(\boldsymbol{d}-\overline{\boldsymbol{d}})\right)^{\Gamma^{u}} d \Gamma_{u}=0, \forall \delta \boldsymbol{d}, \delta \boldsymbol{q}^{\Gamma_{u}} \\
& =-\int_{\Omega}\left(\boldsymbol{n}_{\alpha, \alpha}+\overline{\boldsymbol{n}}^{\Omega}\right) \cdot \delta \boldsymbol{u} d \Omega-\int_{\Omega}\left(\boldsymbol{m}_{\alpha, \alpha}+\boldsymbol{e}_{\alpha}^{r} \times \boldsymbol{n}_{\alpha}+\overline{\boldsymbol{m}}^{\Omega}\right) \cdot \delta \boldsymbol{\theta} d \Omega \\
& +\int_{\Gamma^{t}}^{\Omega}\left(n_{\alpha} \boldsymbol{n}_{\alpha}-\overline{\boldsymbol{n}}^{\Gamma_{t}}\right) \cdot \delta \boldsymbol{u} d \Gamma^{t}+\int_{\Gamma^{t}}\left(n_{\alpha} \boldsymbol{m}_{\alpha}-\overline{\boldsymbol{m}}^{\Gamma_{t}}\right) \cdot \delta \boldsymbol{\theta} d \Gamma^{t} \\
& +\int_{\Gamma^{u}}^{\Gamma^{t}}\left(n_{\alpha} \boldsymbol{n}_{\alpha}-\boldsymbol{n}^{\Gamma_{u}}\right) \cdot \delta \boldsymbol{u} d \Gamma^{u}+\int_{\Gamma^{u}}^{\Gamma^{t}}\left(n_{\alpha} \boldsymbol{m}_{\alpha}-\boldsymbol{m}^{\Gamma_{u}}\right) \cdot \delta \boldsymbol{\theta} d \Gamma^{u} \\
& -\int_{\Gamma_{u}}^{\Gamma_{u}^{u}}\left(\delta \boldsymbol{q}^{\Gamma_{u}} \cdot(\boldsymbol{d}-\overline{\boldsymbol{d}})\right) d \Gamma_{u}=0, \forall \delta \boldsymbol{d}, \delta \boldsymbol{q}^{\Gamma_{u}}
\end{aligned}
$$

de onde pode-se retirar as equações governativas do problema:

$$
\begin{aligned}
\boldsymbol{n}_{\alpha, \alpha}+\overline{\boldsymbol{n}}^{\Omega}=\boldsymbol{O} \\
\boldsymbol{m}_{\alpha, \alpha}+\boldsymbol{e}_{\alpha}^{r} \times \boldsymbol{n}_{\alpha}+\overline{\boldsymbol{m}}^{\Omega}=\boldsymbol{O}
\end{aligned} \quad \text { ou } \quad \boldsymbol{\Delta}_{\alpha}^{*} \boldsymbol{\sigma}_{\alpha}+\overline{\boldsymbol{q}}^{\Omega}=\boldsymbol{O} \mathrm{em} \Omega
$$

com

$$
\begin{aligned}
& \boldsymbol{\Delta}_{\alpha}^{*}= {\left[\begin{array}{cc}
\boldsymbol{I} \frac{\partial}{\partial x_{\alpha}} & \boldsymbol{O} \\
\boldsymbol{E}_{\alpha}^{r} & \boldsymbol{I} \frac{\partial}{\partial x_{\alpha}}
\end{array}\right], } \\
& n_{\alpha} \boldsymbol{n}_{\alpha}-\overline{\boldsymbol{n}}^{\Gamma_{t}}=\boldsymbol{O} \\
& n_{\alpha} \boldsymbol{m}_{\alpha}-\overline{\boldsymbol{m}}^{\Gamma_{t}}=\boldsymbol{O}
\end{aligned} \text { ou } \boldsymbol{\sigma}^{\Gamma}=\overline{\boldsymbol{\sigma}}^{\Gamma} \mathrm{em} \Gamma_{t},
$$

obtido definindo-se 


$$
\boldsymbol{\sigma}^{\Gamma}=\left[\begin{array}{c}
\boldsymbol{n}^{\Gamma} \\
\boldsymbol{m}^{\Gamma}
\end{array}\right]=\nu_{\alpha} \boldsymbol{\sigma}_{\alpha}
$$

e

$$
\begin{aligned}
n_{\alpha} \boldsymbol{n}_{\alpha}-\boldsymbol{n}^{\Gamma_{u}} & =\boldsymbol{O} \\
n_{\alpha} \boldsymbol{m}_{\alpha}-\boldsymbol{m}^{\Gamma_{u}} & =\boldsymbol{O} \text { em } \Gamma_{u}, \\
\boldsymbol{d}-\overline{\boldsymbol{d}} & =\boldsymbol{O}
\end{aligned}
$$

\subsection{Material Isótropo Linear}

A descrição do material será feita com maior rigor no capítulo destinado ao estudo das cascas. Aqui, satisfaz a imposição de uma relação linear entre os esforços generalizados e as deformações generalizadas, na forma

$$
\sigma=D \varepsilon
$$

em que a matriz $D$ é definida por

$$
\begin{aligned}
\boldsymbol{D} & =\left[\begin{array}{ll}
\boldsymbol{D}_{11} & \boldsymbol{D}_{12} \\
\boldsymbol{D}_{21} & \boldsymbol{D}_{22}
\end{array}\right] \\
\boldsymbol{D}_{\alpha \beta} & =\left[\begin{array}{ll}
\frac{\partial \boldsymbol{n}_{\alpha}}{\partial \boldsymbol{\eta}_{\beta}} & \frac{\partial \boldsymbol{n}_{\alpha}}{\partial \boldsymbol{\kappa}_{\beta}} \\
\frac{\partial \boldsymbol{m}_{\alpha}}{\partial \boldsymbol{\eta}_{\beta}} & \frac{\partial \boldsymbol{m}_{\alpha}}{\partial \boldsymbol{\kappa}_{\beta}}
\end{array}\right]
\end{aligned}
$$

\subsection{Aproximação por Multiple Fixed Least Squares}

Assume-se para a aproximação das reações de apoio uma interpolação através de polinômios de Lagrange. Assim, tomando um ponto $\boldsymbol{x}_{u} \in \Gamma_{u}$, cada reação de apoio nesse ponto será aproximada por $\boldsymbol{q}^{\Gamma_{u}}=\Phi^{\Gamma}{ }_{i}\left(\boldsymbol{x}_{u}\right) \boldsymbol{r}_{i}$ onde $\boldsymbol{r}_{i}$ são os parâmetros nodais que definem as reações de apoio generalizadas na fronteira cinemática. 
Os deslocamentos são aproximados por Moving Least Squares como descrito anteriormente, ou seja, $\boldsymbol{d}=\boldsymbol{\Phi}^{\Omega} \boldsymbol{u}$, onde $\Phi^{\Omega}{ }_{i j}=\Phi^{\Omega}{ }_{i}\left(\boldsymbol{x}_{j}\right)$ e $\boldsymbol{x}_{j}$ é cada um dos pontos (ou partículas) usados para descrever a aproximação.

Faz-se a ressalva que o $\boldsymbol{u}$ aqui se refere aos parâmetros nodais de deslocamento e rotação e não mais aos deslocamentos da superfície média.

Assim, a forma fraca discretizada e em forma matricial fica:

$$
\begin{aligned}
\delta W= & \int_{\Omega}\left(\delta \boldsymbol{u}^{T}\left(\boldsymbol{\Delta} \boldsymbol{\Phi}^{\Omega}\right)^{T} \boldsymbol{D} \boldsymbol{\Delta} \boldsymbol{\Phi}^{\Omega} \boldsymbol{u}\right) d \Omega-\int_{\Omega}\left(\delta \boldsymbol{u}^{T} \boldsymbol{\Phi}^{\Omega T} \overline{\boldsymbol{q}}^{\Omega}\right) d \Omega \\
& -\int_{\Gamma_{t}}\left(\delta \boldsymbol{u}^{T} \boldsymbol{\Phi}^{T} \overline{\boldsymbol{q}}^{\Gamma_{t}}\right) d \Gamma_{t}-\int_{\Gamma_{u}}\left(\delta \boldsymbol{u}^{T} \boldsymbol{\Phi}^{\Omega T} \boldsymbol{\Phi}^{T} \boldsymbol{r}\right) d \Gamma_{u} \\
& -\int_{\Gamma_{u}}\left(\delta \boldsymbol{r}^{T} \boldsymbol{\Phi}^{T T} \boldsymbol{\Phi}^{\Omega} \boldsymbol{u}\right) d \Gamma_{u}+\int_{\Gamma_{u}}\left(\delta \boldsymbol{r}^{T} \boldsymbol{\Phi}^{T} \overline{\boldsymbol{d}}\right) d \Gamma_{u}=0, \forall \delta \boldsymbol{u}, \delta \boldsymbol{r}
\end{aligned}
$$

e o sistema discretizado de equações é

$$
\left[\begin{array}{cc}
\mathbf{K} & \mathbf{G} \\
\mathbf{G}^{\mathbf{T}} & \mathbf{0}
\end{array}\right]\left\{\begin{array}{l}
\mathbf{u} \\
\mathbf{r}
\end{array}\right\}=\left\{\begin{array}{l}
\mathbf{f} \\
\mathbf{q}
\end{array}\right\}
$$

com

$$
\begin{aligned}
\mathbf{K} & =\int_{\Omega}\left(\boldsymbol{\Delta} \boldsymbol{\Phi}^{\Omega}\right)^{T} \boldsymbol{D} \boldsymbol{\Delta} \boldsymbol{\Phi}^{\Omega} d \Omega \\
\mathbf{G} & =-\int_{\Gamma_{u}} \boldsymbol{\Phi}^{\Omega T} \boldsymbol{\Phi}^{T} d \Gamma_{u} \\
\mathbf{f} & =\int_{\Omega}\left(\boldsymbol{\Phi}^{\Omega T} \overline{\boldsymbol{q}}^{\Omega}\right) d \Omega+\int_{\Gamma_{t}} \boldsymbol{\Phi}^{\Omega T} \overline{\boldsymbol{q}}^{\Gamma_{t}} d \Gamma_{t} . \\
\mathbf{q} & =-\int_{\Gamma_{u}} \boldsymbol{\Phi}^{\Gamma T} \overline{\boldsymbol{d}} d \Gamma_{u}
\end{aligned}
$$




\subsection{Exemplos Numéricos}

Para ilustrar a formulação apresentada, esta foi codificada em MATLAB de forma a permitir efetuar-se simulações numéricas.

\subsubsection{Viga engastada com carregamento na extremidade}

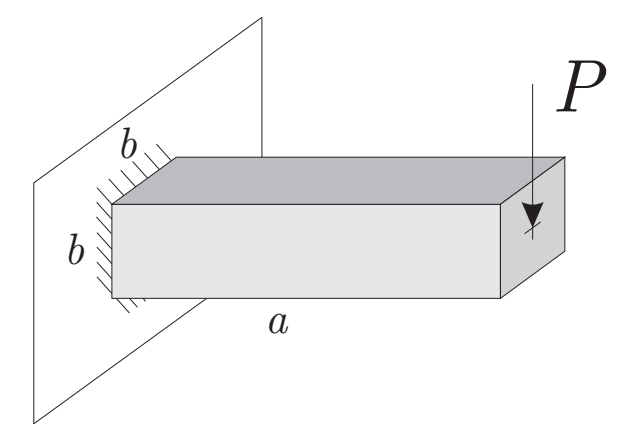

Figura 7 - Viga em balanço com carga na extremidade

A viga com carregamento na extremidade ilustrada na Figura 7 pode ser tratada nessa formulação de duas formas: um placa no plano $\boldsymbol{e}_{1}^{r} \times \boldsymbol{e}_{2}^{r}$ com o carregamento na direção $\boldsymbol{e}_{3}^{r}$, conforme a Figura 8 ou como um chapa (elasticidade bidimensional, com estado plano de tensões) no plano $\boldsymbol{e}_{1}^{r} \times \boldsymbol{e}_{2}^{r}$ com carregamento na direção $\boldsymbol{e}_{2}^{r}$, como na Figura 9.

Para uma mais fácil comparação das duas possibilidades apresentadas, tomamos uma viga com secção quadrada e de comprimento bem maior que as outras dimensões, de forma a podermos comparar os resultados com a resposta provida pela teoria de barras de Timoshenko. Assim, as dimensões adotadas foram

$$
a=20, b=2 \text { e } P=1,
$$

e as propriedades do material adotado

$$
E=10^{6} \text { e } \nu=0,3
$$


em um sistema de unidades consistente. A resposta analítica para o deslocamento do ponto de aplicação da carga, dada por Timoshenko [6] é

$$
\frac{P a^{3}}{3 E I}+\frac{P a}{G A^{\prime}}, I=\frac{b h^{3}}{12}
$$

$b$ e $h$ sendo as dimensões da seção transversal e $A^{\prime}$ a área equivalente de cisalhamento, aqui tomada como $5 / 6 b^{2}$.

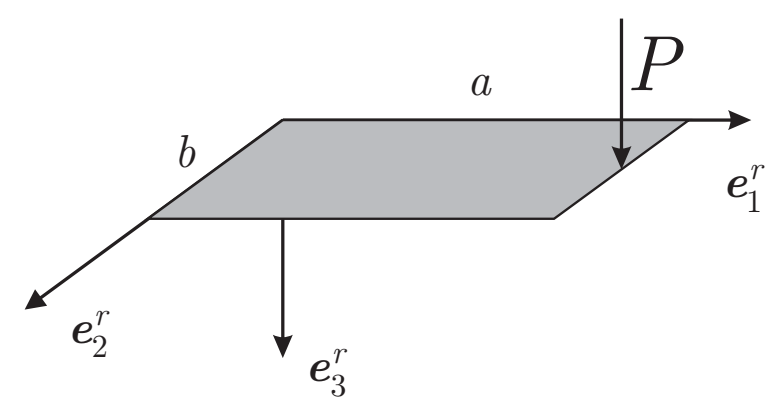

Figura 8 - Viga em balanço modelada como placa

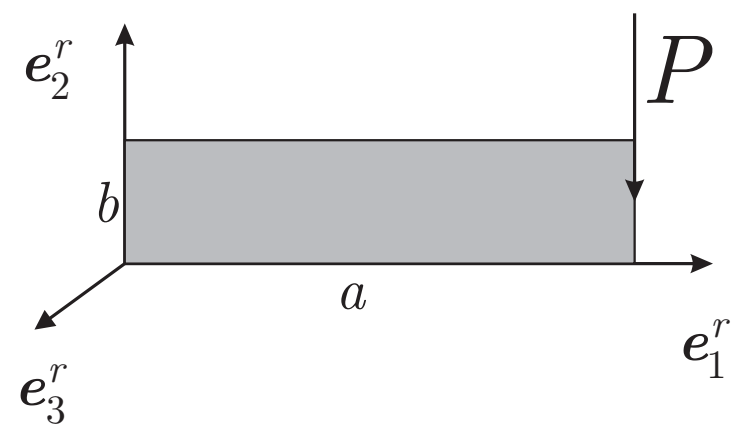

Figura 9 - Viga em balanço modelada como chapa

Em ambas as simulações, foi usada uma função peso do tipo spline ajustável

$$
\left(1-r^{2}\right)^{3}
$$

em que $r$ é a distância normalizada do ponto de interesse à partícula. O suporte das partículas foi maximizado por $d_{\max }=1,5$, a integração foi feita com 250 células de integração, cada 
uma contendo três pontos de integração, pela regra de Gauss, em cada direção $\boldsymbol{e}_{\alpha}^{r}$, conforme a Figura 10.

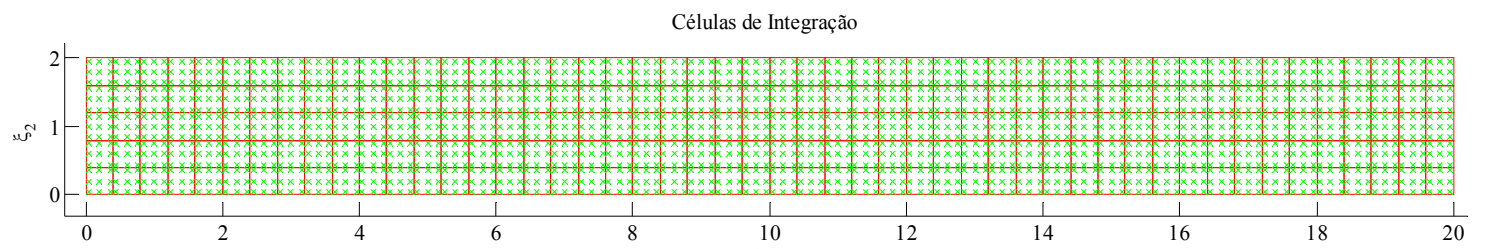

Figura 10 - Células de integração para viga em balanço

Foram usadas quatro distribuições de partículas para cada simulação, com 63, 124, 205 e 306 partículas, conforme a Figura 11.
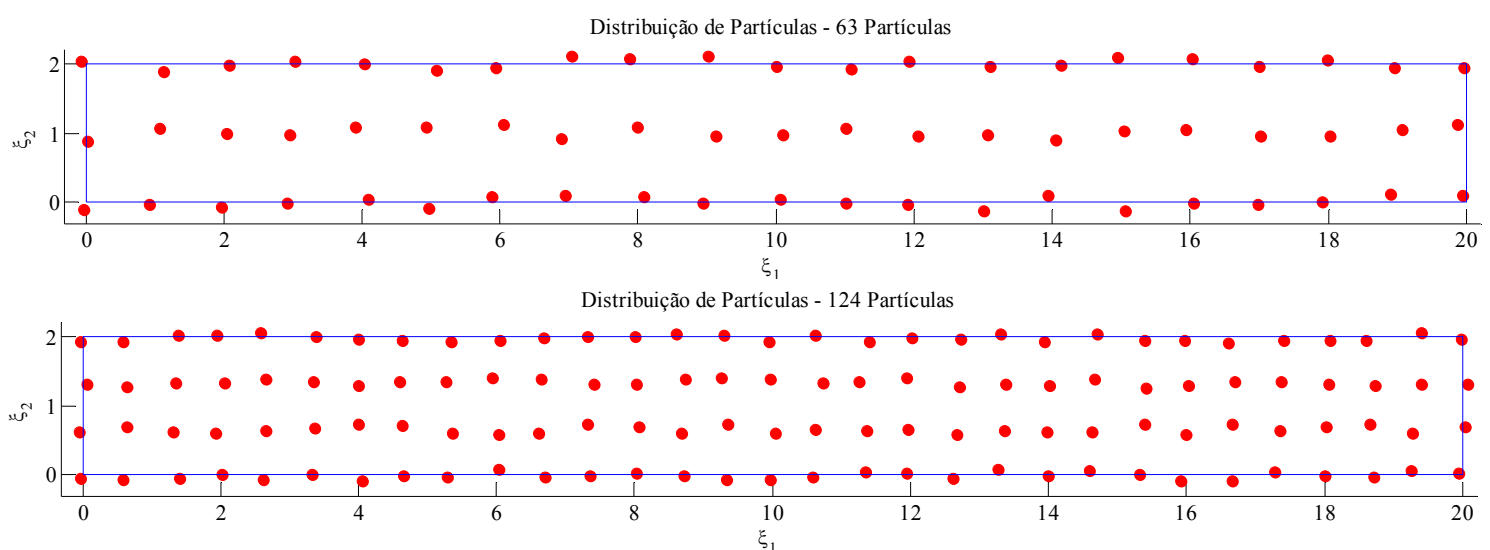

Distribuição de Partículas - 205 Partículas

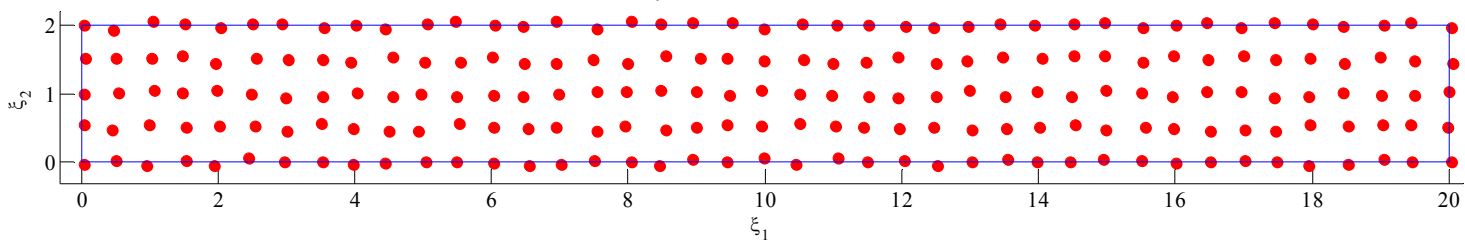

Distribuição de Partículas - 205 Partículas

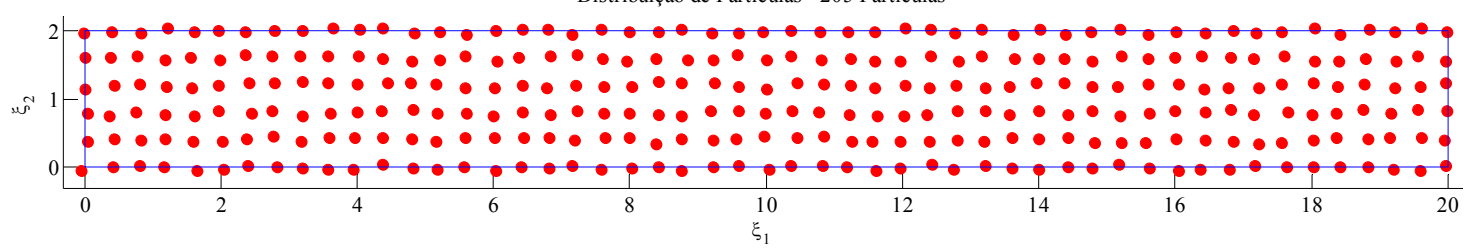

Figura 11 - Distribuição de Partículas para Viga (chapa e placa)

Para a confecção da distribuição de partículas, foi adicionada à distribuição uniforme uma perturbação pseudorrandômica da posição das partículas de forma que não fícassem 
alinhadas. O alinhamento das partículas gera dependências lineares nas funções de forma, de maneira que é necessário um domínio maior das nuvens para produzir uma resposta satisfatória.

O resultado do deslocamento do ponto de aplicação da carga (vertical ou horizontal de acordo com forma de simulação), normalizado pela resposta analítica, para as várias discretizações está apresentado na Figura 12.

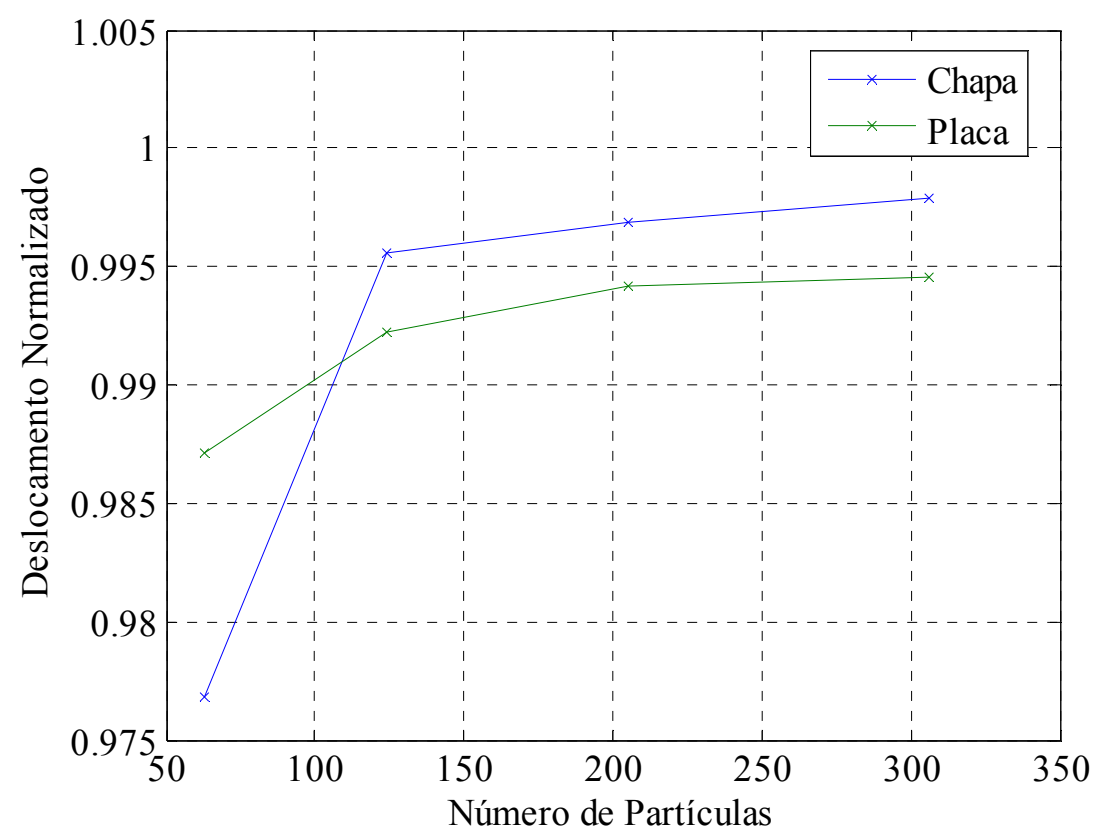

Figura 12 - Viga em balanço com carga na extremidade - Resultados

Os resultados obtidos foram satisfatoriamente próximos à resposta dada pela teoria de barra de Timoshenko. Vale a pena ressaltar que se tratam de três diferentes teorias estruturais, três diferentes modelos sendo resolvidos, daí a diferença presente entre as soluções.

\subsubsection{Placa Carregada Uniformemente}

O exemplo anterior gera bons resultados para malhas relativamente grosseiras. A fim de se ter uma melhor perspectiva do comportamento da resposta com o refinar da distribuição de partículas, propõe-se o estudo de uma placa quadrada com carregamento uniforme perpendicular, como ilustrado na Figura 13. A placa encontra-se simplesmente apoiada em 
seus bordos. O apoio é tomado como do tipo hard, ou seja, são restritos os deslocamentos verticais e as rotações perpendiculares à borda. Há ainda restrições de deslocamento normal a duas bordas não opostas, a fim de se evitar movimentos de corpo rígido.

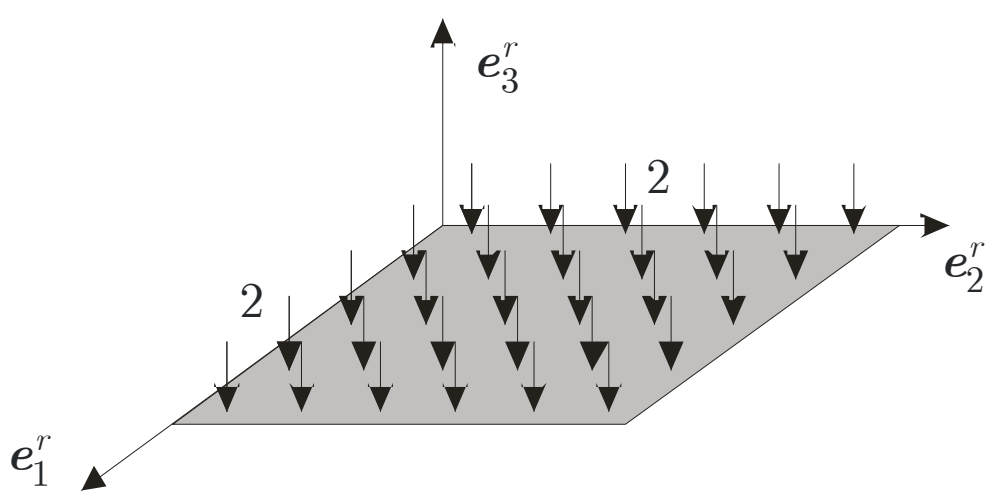

Figura 13 - Placa carregada uniformemente

As dimensões da placa superfície de referência da placa são tomadas como constante $(2 \times 2)$ e a análise é feita para diversas espessuras. A carga é tomada como $6,25 \times 10^{4} \times$ espessura, de forma a adimensionalizar o problema. O material tem módulo de elasticidade $E=2,1 \times 10^{9}$ e coeficiente de Poisson $\nu=0,3$.

Os resultados são comparados com a solução analítica dada em Wang, Reddy e Lee [10], constituída de uma série de somatórios, usando os 101 primeiros termos.

Mais uma vez foi adotada a função peso exponencial e o domínio foi majorado pelo fator $d_{\max }=1,75$. A base polinomial adotada foi um polinômio completo de ordem 4 em $e_{1}^{r} \mathrm{e}$ $\boldsymbol{e}_{2}^{r}$. Simulações com polinômios de ordem inferior demonstraram travamento por cortante.

Uma malha de fundo, estruturada, foi gerada em cada simulação para a integração, feita com cinco pontos de Gauss em cada direção. As partículas foram tomadas como os nós dessa malha. A Figura 14 ilustra uma delas. 

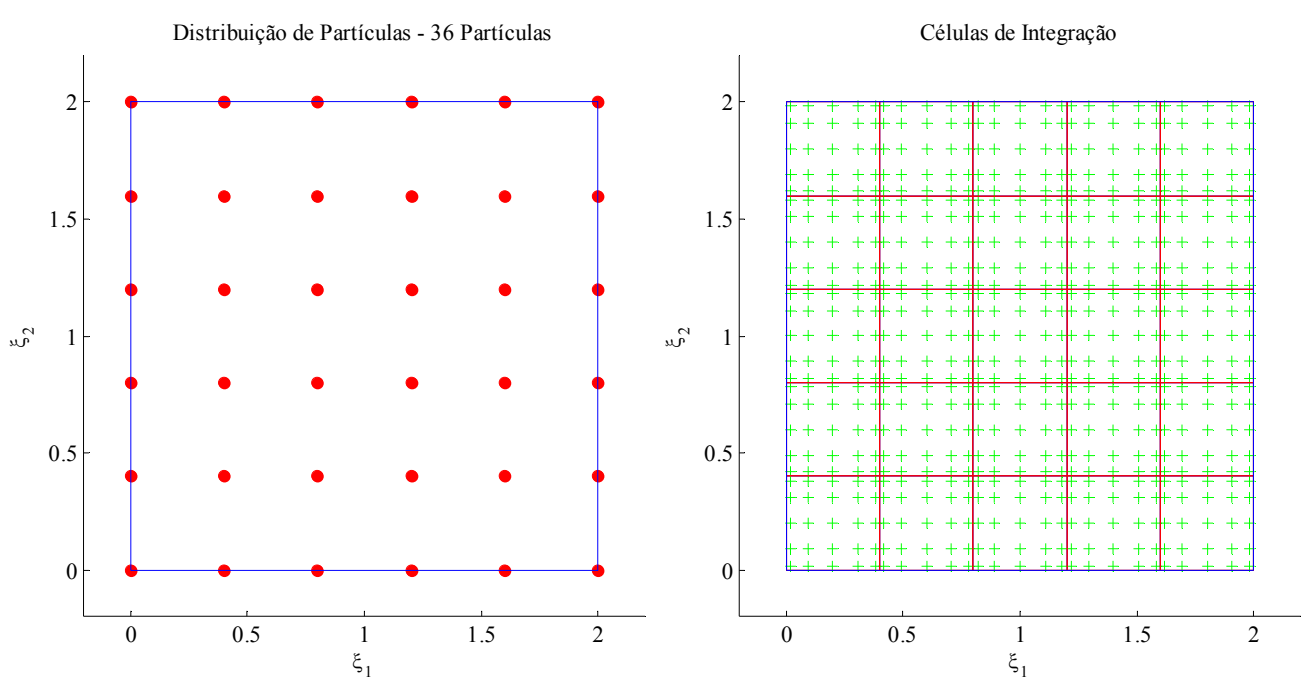

Figura 14 - Distribuição de Partículas e Células de Integração

Assim, o refinamento da malha melhora tanto a aproximação, pelo número de partículas, quanto a integração, pelo número de células de integração. Os resultados obtidos com diferentes discretizações para diferentes espessuras de placas são mostrados na Figura 15.

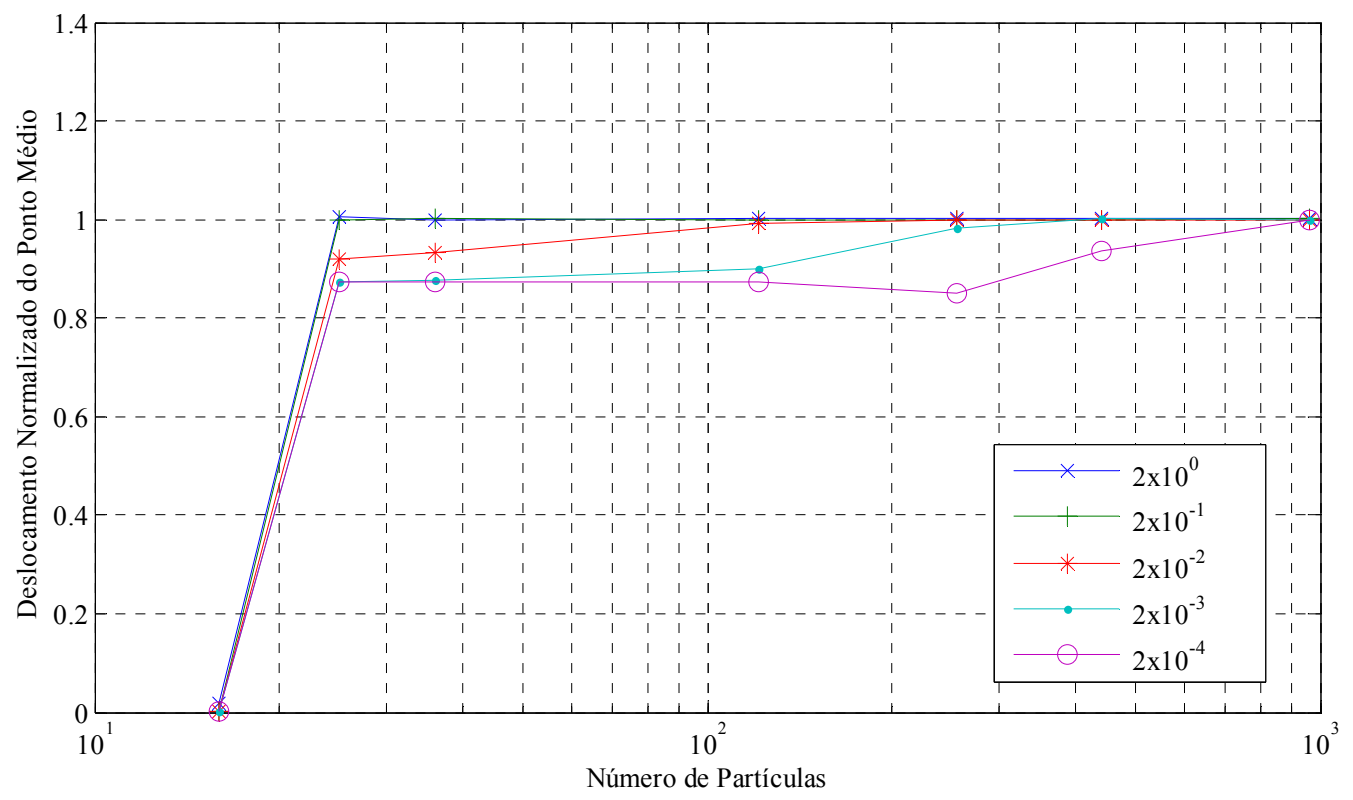

Figura 15 - Deslocamento normalizado da placa x Número de Partículas

Mais uma vez os resultados são satisfatórios, até para placas bastante finas, com relação espessura / borda da ordem de $10^{-4}$. Nessa mesma placa, faz-se interessante a observação do ponto onde a resposta foi pior em relação à distribuição anterior. Isso pode ser explicado no 
campo dos métodos sem malha pela consideração de que o espaço de aproximação gerado por uma distribuição com mais partículas não necessariamente contém o espaço gerado por outra distribuição com menos pontos.

Para a malha apresentada (36 partículas), o resultado obtido para os deslocamentos se apresentam na Figura 16, onde os deslocamentos foram exagerados para poderem ser mais bem observados.

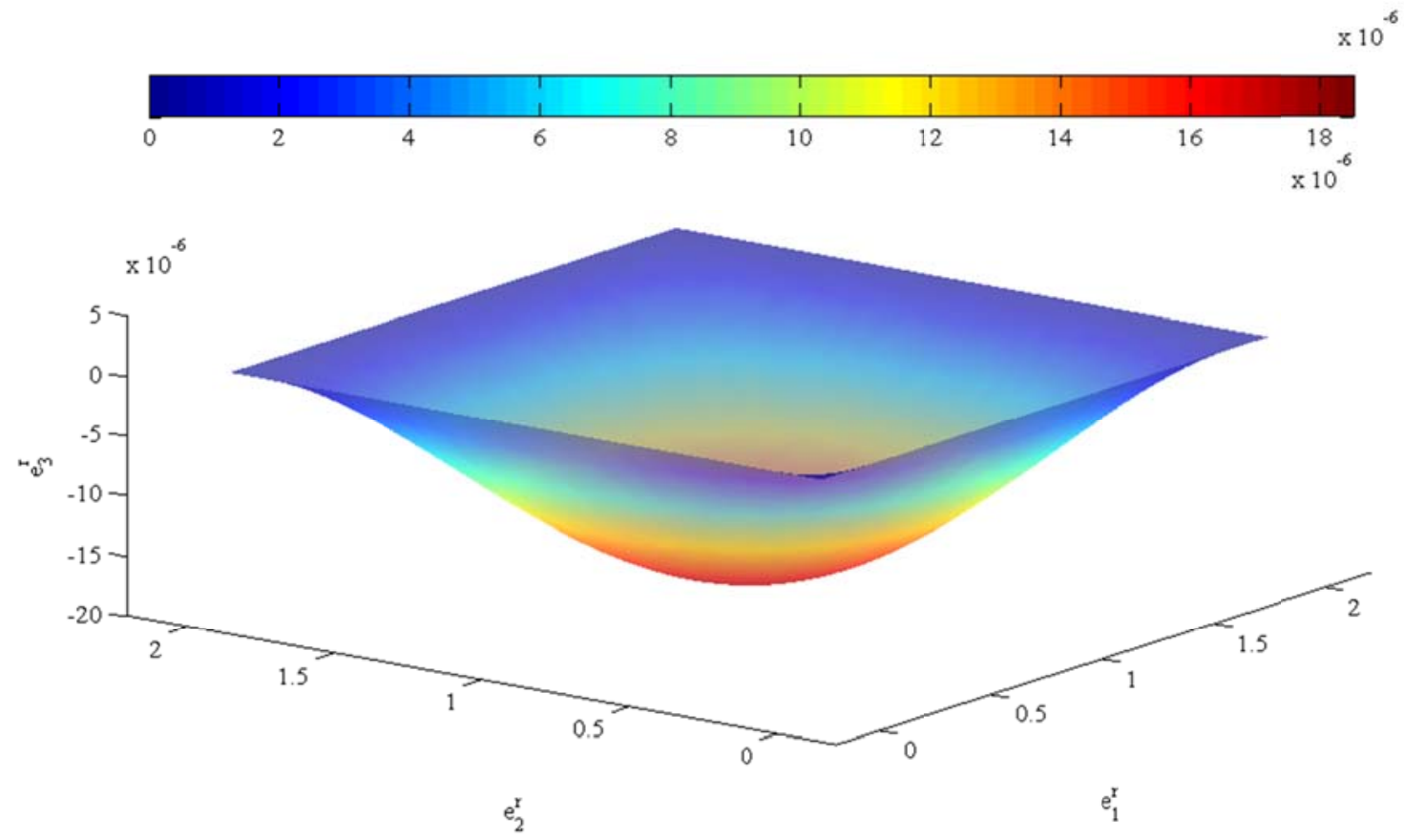

Figura 16 - Configuração deformada da placa (eixo $e_{3}^{r}$ fora de escala) 
As deformações generalizadas obtidas na mesma simulação estão na Figura 17, onde fica claro o grau de continuidade obtido com o $M L S$.
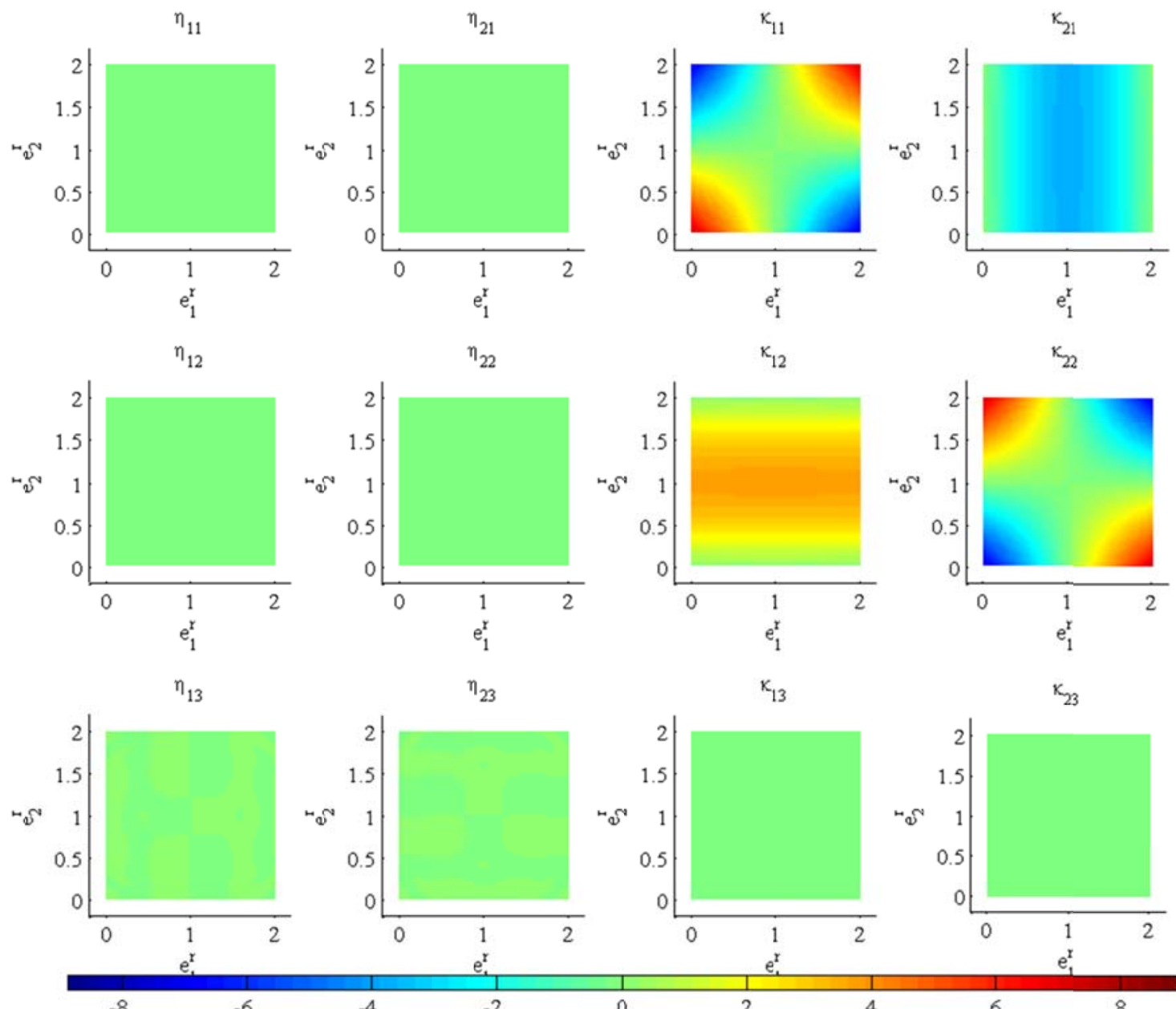

Figura 17 - Deformações da Placa 


\section{TEORIA DE CASCAS GEOMETRICAMENTE EXATA}

O presente capítulo busca descrever a teoria de cascas desenvolvida por Pimenta e Campello em [1] e implementada em métodos sem malha por Tiago em [2]. Sua formulação combina as ideias de Pimenta [8] e de Campello, Pimenta e Wriggers [11] para cascas inicialmente planas com as presentes em Pimenta [9] para barras curvas, e impõe sobre a casca sua configuração inicial na forma de uma deformação livre de tensões, a partir da qual se dará o movimento real da mesma.

Primeiramente, a teoria é descrita sem consideração alguma com relação à magnitude dos deslocamentos, rotações e deformações da casca. Somente no próximo capítulo tais hipóteses serão consideradas.

Seja a casca como mostrada na figura abaixo.

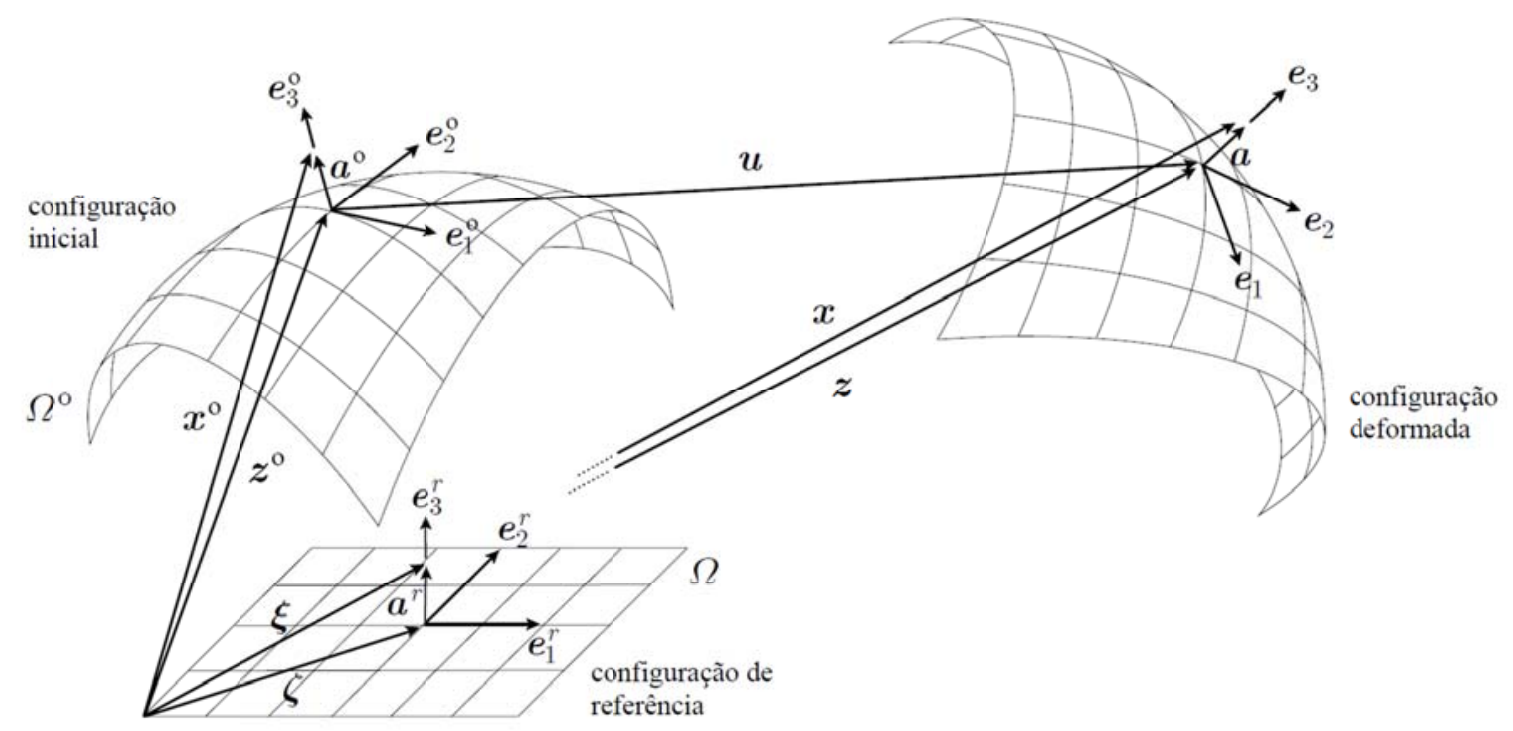

Figura 18 - Configurações de referência, inicial e final da casca (Retirada de Campello [12])

A casca é descrita em sua configuração plana de referência. Nessa configuração, define-se a base $\boldsymbol{e}_{i}^{r}$, tal que os versores $\boldsymbol{e}_{\alpha}^{r}$ estão no plano da superfície de referência da casca e $\boldsymbol{e}_{3}^{r}$ no sentido de sua espessura. A posição de um ponto da casca nessa configuração é dada por 


$$
\boldsymbol{\xi}=\boldsymbol{\zeta}+\boldsymbol{a}^{r}
$$

onde $\zeta=\xi_{\alpha} e_{\alpha}$ define a projeção do ponto material na superfície de referência $\Omega^{r}$ e o vetor $\boldsymbol{a}^{r}=\xi_{3} \boldsymbol{e}_{3}^{r}$, chamado diretor, o posiciona ao longo da espessura. Assim, $\xi_{3} \in\left[-h^{b}, h^{t}\right]$ é a coordenada na espessura da casca. A espessura total na configuração de referência é $h=h^{b}+h^{t}$. A tríade $\left\{\xi_{1}, \xi_{2}, \xi_{3}\right\}$ forma um sistema cartesiano de três dimensões.

\subsection{Transformação Inicial}

A configuração inicial da casca é descrita por uma deformação livre de tensões. A superfície de referência será mapeada a partir de sua configuração plana por

$$
\boldsymbol{z}^{o}=\boldsymbol{z}^{o}\left(\xi_{\alpha}\right)
$$

O diretor será rotacionado sem sofrer alteração no seu comprimento. Esta rotação pode ser descrita pelo tensor $\boldsymbol{Q}^{o}$. O diretor inicial será, então

$$
\boldsymbol{a}^{o}=\boldsymbol{Q}^{o} \boldsymbol{a}^{r}
$$

Essa deformação é admitida do tipo Kirchhoff-Love, de forma que o diretor rotacionado permanece perpendicular à superfície média. A base $\boldsymbol{e}_{i}^{r}$ segue o diretor, tornando-se $\boldsymbol{e}_{i}^{o}=\boldsymbol{Q}^{o} \boldsymbol{e}_{i}^{r}$. Para definir esta rotação, toma-se $\boldsymbol{e}_{1}^{o}$ tal que acompanhe a curvatura da superfície média em relação a $\xi_{1}$,

$$
\boldsymbol{e}_{1}^{o}=\frac{\boldsymbol{z}_{, 1}^{o}}{\left\|\boldsymbol{z}_{, 1}^{o}\right\|}
$$

$\boldsymbol{e}_{3}^{o}$ segue o diretor, ou seja, é tomado perpendicular a ambas as curvaturas da casca:

$$
\boldsymbol{e}_{3}^{o}=\frac{\boldsymbol{z}_{, 1}^{o} \times \boldsymbol{z}_{, 2}^{o}}{\left\|\boldsymbol{z}_{, 1}^{o} \times \boldsymbol{z}_{, 2}^{o}\right\|},
$$


e $e_{2}^{o}$ comporá a base ortonormal sendo simultaneamente perpendicular a $e_{1}^{o}$ e $e_{3}^{o}$ :

$$
\boldsymbol{e}_{2}^{o}=\boldsymbol{e}_{3}^{o} \times \boldsymbol{e}_{1}^{o}
$$

Com seus vetores componentes, a rotação inicial pode ser expressa por

$$
\boldsymbol{Q}^{o}=\boldsymbol{e}_{i}^{o} \otimes \boldsymbol{e}_{i}^{r}
$$

Pode-se ter um melhor entendimento do posicionamento dos vetores $\boldsymbol{e}_{i}^{o}$ quando das definições da transformação inicial na subseção 4.1.1.

Serão úteis as derivações de $\boldsymbol{e}_{i, \alpha}^{o}$. Prova-se que

$$
\left\|\boldsymbol{z}_{, 1}^{o}\right\|_{, \alpha}=\left(\sqrt{\boldsymbol{z}_{, 1}^{o} \cdot \boldsymbol{z}_{, 1}^{o}}\right)_{, \alpha}=\frac{1}{2} \frac{\left(\boldsymbol{z}_{, 1}^{o} \cdot \boldsymbol{z}_{, 1 \alpha}^{o}+\boldsymbol{z}_{, 1 \alpha}^{o} \cdot \boldsymbol{z}_{, 1}^{o}\right)}{\sqrt{\boldsymbol{z}_{, 1}^{o} \cdot \boldsymbol{z}_{, 1}^{o}}}=\frac{\boldsymbol{z}_{, 1}^{o} \cdot \boldsymbol{z}_{, 1 \alpha}^{o}}{\left\|\boldsymbol{z}_{, 1}^{o}\right\|}
$$

Logo,

$$
\begin{aligned}
\boldsymbol{e}_{1, \alpha}^{o} & =\left(\frac{\boldsymbol{z}_{, 1}^{o}}{\left\|\boldsymbol{z}_{, 1}^{o}\right\|}=\frac{\boldsymbol{z}_{, 1 \alpha}^{o}}{\left\|\boldsymbol{z}_{, 1}^{o}\right\|}-\frac{\boldsymbol{z}_{, 1}^{o}}{\left\|\boldsymbol{z}_{, 1}^{o}\right\|^{2}}\left\|\boldsymbol{z}_{, 1}^{o}\right\|_{, \alpha}=\frac{\boldsymbol{z}_{, 1 \alpha}^{o}}{\left\|\boldsymbol{z}_{, 1}^{o}\right\|}-\frac{\boldsymbol{z}_{, 1}^{o} \cdot \boldsymbol{z}_{, 1 \alpha}^{o}}{\left\|\boldsymbol{z}_{, 1}^{o}\right\|^{3}} \boldsymbol{z}_{, 1}^{o}=\right. \\
& \left.=\frac{1}{\left\|\boldsymbol{z}_{, 1}^{o}\right\|} \boldsymbol{z}_{, 1 \alpha}^{o}-\frac{\boldsymbol{z}_{, 1}^{o} \otimes \boldsymbol{z}_{, 1}^{o}}{\left\|\boldsymbol{z}_{, 1}^{o}\right\|^{2}} \boldsymbol{z}_{, 1 \alpha}^{o}\right)=\frac{1}{\left\|\boldsymbol{z}_{, 1}^{o}\right\|}\left(\boldsymbol{I}-\boldsymbol{e}_{1}^{o} \otimes \boldsymbol{e}_{1}^{o}\right) \boldsymbol{z}_{, 1 \alpha}^{o}
\end{aligned}
$$




$$
\begin{aligned}
& \boldsymbol{e}_{3, \alpha}^{o}=\left(\frac{\boldsymbol{z}_{, 1}^{o} \times \boldsymbol{z}_{, 2}^{o}}{\left\|\boldsymbol{z}_{, 1}^{o} \times \boldsymbol{z}_{, 2}^{o}\right\|}\right)_{, \alpha} \\
& =\frac{\boldsymbol{z}_{, 1 \alpha}^{o} \times \boldsymbol{z}_{, 2}^{o}+\boldsymbol{z}_{, 1}^{o} \times \boldsymbol{z}_{, 2 \alpha}^{o}}{\left\|\boldsymbol{z}_{, 1}^{o} \times \boldsymbol{z}_{, 2}^{o}\right\|} \\
& -\frac{\boldsymbol{z}_{, 1}^{o} \times \boldsymbol{z}_{, 2}^{o}}{\left\|\boldsymbol{z}_{, 1}^{o} \times \boldsymbol{z}_{, 2}^{o}\right\|^{3}}\left(\boldsymbol{z}_{, 1}^{o} \times \boldsymbol{z}_{, 2}^{o}\right) \cdot\left(\boldsymbol{z}_{, 1 \alpha}^{o} \times \boldsymbol{z}_{, 2}^{o}+\boldsymbol{z}_{, 1}^{o} \times \boldsymbol{z}_{, 2 \alpha}^{o}\right), \mathrm{e} \\
& =\frac{1}{\left\|\boldsymbol{z}_{, 1}^{o} \times \boldsymbol{z}_{, 2}^{o}\right\|}\left(\boldsymbol{z}_{, 1 \alpha}^{o} \times \boldsymbol{z}_{, 2}^{o}+\boldsymbol{z}_{, 1}^{o} \times \boldsymbol{z}_{, 2 \alpha}^{o}\right. \\
& \left.-\frac{\left(\boldsymbol{z}_{, 1}^{o} \times \boldsymbol{z}_{, 2}^{o}\right) \otimes\left(\boldsymbol{z}_{, 1}^{o} \times \boldsymbol{z}_{, 2}^{o}\right)}{\left\|\boldsymbol{z}_{, 1}^{o} \times \boldsymbol{z}_{, 2}^{o}\right\|^{2}}\left(\boldsymbol{z}_{, 1 \alpha}^{o} \times \boldsymbol{z}_{, 2}^{o}+\boldsymbol{z}_{, 1}^{o} \times \boldsymbol{z}_{, 2 \alpha}^{o}\right)\right) \\
& =\frac{1}{\left\|\boldsymbol{z}_{, 1}^{o} \times \boldsymbol{z}_{, 2}^{o}\right\|}\left(\boldsymbol{I}-\boldsymbol{e}_{3}^{o} \otimes \boldsymbol{e}_{3}^{o}\right)\left(\boldsymbol{z}_{, 1}^{o} \times \boldsymbol{z}_{, 2 \alpha}^{o}-\boldsymbol{z}_{, 2}^{o} \times \boldsymbol{z}_{, 1 \alpha}^{o}\right) \\
& \boldsymbol{e}_{2, \alpha}^{o}=\left(\boldsymbol{e}_{3}^{o} \times \boldsymbol{e}_{1}^{o}\right)_{, \alpha}=\boldsymbol{e}_{3, \alpha}^{o} \times \boldsymbol{e}_{1}^{o}+\boldsymbol{e}_{3}^{o} \times \boldsymbol{e}_{1, \alpha}^{o} .
\end{aligned}
$$

O gradiente da transformação inicial pode ser escrito na forma

$$
\begin{aligned}
\boldsymbol{F}^{o} & =\frac{\partial \boldsymbol{x}^{o}}{\partial \boldsymbol{\xi}}=\frac{\partial \boldsymbol{x}^{o}}{\partial \xi_{\alpha}} \otimes \boldsymbol{e}_{\alpha}^{r}+\frac{\partial \boldsymbol{x}^{o}}{\partial \xi_{3}} \otimes \boldsymbol{e}_{3}^{r} \\
& =\left(\boldsymbol{z}_{, \alpha}^{o}+\boldsymbol{Q}_{, \alpha}^{o} \boldsymbol{Q}^{o T} \boldsymbol{a}^{o}\right) \otimes \boldsymbol{e}_{\alpha}^{r}+\boldsymbol{a}^{o \prime} \otimes \boldsymbol{e}_{3}^{r} \\
& =\left(\boldsymbol{z}_{, \alpha}^{o}+\boldsymbol{Q}_{, \alpha}^{o} \boldsymbol{Q}^{o T} \boldsymbol{a}^{o}\right) \otimes \boldsymbol{e}_{\alpha}^{r}+\boldsymbol{Q}^{o} \boldsymbol{e}_{3}^{r} \otimes \boldsymbol{e}_{3}^{r} \\
& =\left(\boldsymbol{z}_{, \alpha}^{o}-\boldsymbol{Q}^{o} \boldsymbol{e}_{\alpha}^{r}+\boldsymbol{Q}_{, \alpha}^{o} \boldsymbol{Q}^{o T} \boldsymbol{a}^{o}\right) \otimes \boldsymbol{e}_{\alpha}^{r}+\boldsymbol{Q}^{o}\left(\boldsymbol{e}_{\alpha}^{r} \otimes \boldsymbol{e}_{\alpha}^{r}+\boldsymbol{e}_{3}^{r} \otimes \boldsymbol{e}_{3}^{r}\right) \\
& =\left(\boldsymbol{\eta}_{\alpha}^{o}+\boldsymbol{K}_{\alpha}^{o} \boldsymbol{a}^{o}\right) \otimes \boldsymbol{e}_{\alpha}^{r}+\boldsymbol{Q}^{o}
\end{aligned}
$$

onde foram introduzidos os vetores de deformações de membrana

$$
\boldsymbol{\eta}_{\alpha}^{o}=\boldsymbol{z}_{, \alpha}^{o}-\boldsymbol{Q}^{o} \boldsymbol{e}_{\alpha}^{r}
$$

e os tensores antissimétricos relacionados com as curvaturas iniciais da casca 


$$
\begin{aligned}
\boldsymbol{K}_{\alpha}^{o} & =\boldsymbol{Q}_{, \alpha}^{o} \boldsymbol{Q}^{o T} \\
& =\left(\boldsymbol{e}_{i, \alpha}^{o} \otimes \boldsymbol{e}_{i}^{r}\right)\left(\boldsymbol{e}_{j}^{o} \otimes \boldsymbol{e}_{j}^{r}\right)^{T} \\
& =\left(\boldsymbol{e}_{i, \alpha}^{o} \otimes \boldsymbol{e}_{i}^{r}\right)\left(\boldsymbol{e}_{j}^{r} \otimes \boldsymbol{e}_{j}^{o}\right)=\left(\boldsymbol{e}_{i, \alpha}^{o} \otimes \boldsymbol{e}_{j}^{r}\right)\left(\boldsymbol{e}_{i}^{r} \cdot \boldsymbol{e}_{j}^{r}\right) \\
& =\boldsymbol{e}_{i, \alpha}^{o} \otimes \boldsymbol{e}_{i}^{o}
\end{aligned}
$$

que têm seus vetores axiais $\kappa_{\alpha}^{o}$ dados por

$$
\boldsymbol{\kappa}_{\alpha}^{o}=\operatorname{axial}\left(\boldsymbol{K}_{\alpha}^{o}\right)=\left(\boldsymbol{e}_{2, \alpha}^{o} \cdot \boldsymbol{e}_{3}^{o}\right) \boldsymbol{e}_{1}^{o}+\left(\boldsymbol{e}_{3, \alpha}^{o} \cdot \boldsymbol{e}_{1}^{o}\right) \boldsymbol{e}_{2}^{o}+\left(\boldsymbol{e}_{1, \alpha}^{o} \cdot \boldsymbol{e}_{2}^{o}\right) \boldsymbol{e}_{1}^{o}
$$

As deformações iniciais em qualquer ponto da casca podem ser recolhidas, então, nos vetores

$$
\gamma_{\alpha}^{o}=\boldsymbol{\eta}_{\alpha}^{o}+\boldsymbol{\kappa}_{\alpha}^{o} \times \boldsymbol{a}^{o}
$$

tal que o gradiente da transformação inicial possa ser escrito como

$$
\boldsymbol{F}^{o}=\left(\boldsymbol{\eta}_{\alpha}^{o}+\boldsymbol{\kappa}_{\alpha}^{o} \times \boldsymbol{a}^{o}\right) \otimes \boldsymbol{e}_{\alpha}^{r}+\boldsymbol{Q}^{o}=\boldsymbol{\gamma}_{\alpha}^{o} \otimes \boldsymbol{e}_{\alpha}^{r}+\boldsymbol{Q}^{o}
$$

Para a elaboração de uma teoria objetiva, bem como uma mais fácil definição das equações constitutivas, é necessário trabalhar-se com grandezas objetivas. Os vetores $\gamma_{\alpha}^{o}$ são afetados por movimentos de corpo rígido. Sua utilização como deformações generalizadas não é pertinente às definições do material, em contraste com os vetores retro-rotacionados $\gamma_{\alpha}^{o r}$ dados por

$$
\begin{aligned}
\boldsymbol{\gamma}_{\alpha}^{o r} & =\boldsymbol{Q}^{o T} \boldsymbol{\gamma}_{\alpha}^{o}=\boldsymbol{Q}^{o T}\left(\boldsymbol{\eta}_{\alpha}^{o}+\boldsymbol{\kappa}_{\alpha}^{o} \times \boldsymbol{a}^{o}\right) \\
& =\boldsymbol{Q}^{o T} \boldsymbol{\eta}_{\alpha}^{o}+\left(\boldsymbol{Q}^{o T} \boldsymbol{\kappa}_{\alpha}^{o}\right) \times\left(\boldsymbol{Q}^{o T} \boldsymbol{a}^{o}\right) \\
& =\boldsymbol{\eta}_{\alpha}^{o r}+\boldsymbol{\kappa}_{\alpha}^{o r} \times \boldsymbol{a}^{r}
\end{aligned}
$$

É interessante notar que de (4.9)-(4.11) e (4.18), 


$$
\begin{aligned}
\boldsymbol{\gamma}_{\alpha}^{o r} \cdot \boldsymbol{e}_{3}^{r} & =\left(\boldsymbol{\eta}_{\alpha}^{o r}+\boldsymbol{\kappa}_{\alpha}^{o r} \times \boldsymbol{a}^{r}\right) \cdot \boldsymbol{e}_{3}^{r}=\boldsymbol{\eta}_{\alpha}^{o r} \cdot \boldsymbol{e}_{3}^{r}+\boldsymbol{\kappa}_{\alpha}^{o r} \times \boldsymbol{a}^{r} \cdot \boldsymbol{e}_{3}^{r} \\
& =\left(\boldsymbol{Q}^{o T} \boldsymbol{z}_{, \alpha}^{o}-\boldsymbol{e}_{\alpha}^{r}\right) \cdot \boldsymbol{e}_{3}^{r}+\boldsymbol{\kappa}_{\alpha}^{o r} \cdot \boldsymbol{a}^{r} \times \boldsymbol{e}_{3}^{r}=\boldsymbol{z}_{, \alpha}^{o} \cdot \boldsymbol{e}_{3}^{o} \\
& =\boldsymbol{z}_{, \alpha}^{o} \cdot \frac{\boldsymbol{z}_{, 1}^{o} \times \boldsymbol{z}_{, 2}^{o}}{\left\|\boldsymbol{z}_{, 1}^{o} \times \boldsymbol{z}_{, 2}^{o}\right\|}=0
\end{aligned}
$$

ou seja, as deformações generalizadas não têm componentes fora do plano da placa, o que é uma característica típica de uma transformação do tipo Kirchhoff-Love.

As deformações de membrana e as curvaturas retro-rotacionadas que aparecem em (4.18) são dados por

$$
\begin{aligned}
& \boldsymbol{\eta}_{\alpha}^{o r}=\boldsymbol{Q}^{o T} \boldsymbol{\eta}_{\alpha}^{o}=\boldsymbol{Q}^{o T} \boldsymbol{z}_{, \alpha}^{o}-\boldsymbol{e}_{\alpha}^{r} \\
& \boldsymbol{\kappa}_{\alpha}^{o r}=\boldsymbol{Q}^{o T} \boldsymbol{\kappa}_{\alpha}^{o}=\left(\boldsymbol{e}_{2, \alpha}^{o} \cdot \boldsymbol{e}_{3}^{o}\right) \boldsymbol{e}_{1}^{r}+\left(\boldsymbol{e}_{3, \alpha}^{o} \cdot \boldsymbol{e}_{1}^{o}\right) \boldsymbol{e}_{2}^{r}+\left(\boldsymbol{e}_{1, \alpha}^{o} \cdot \boldsymbol{e}_{2}^{o}\right) \boldsymbol{e}_{3}^{r}
\end{aligned}
$$

O gradiente da transformação pode então ser expresso como uma função das deformações generalizadas retro-rotacionadas

$$
\begin{aligned}
\boldsymbol{F}^{o} & =\gamma_{\alpha}^{o} \otimes \boldsymbol{e}_{\alpha}^{r}+\boldsymbol{Q}^{o}=\left(\boldsymbol{Q}^{o} \boldsymbol{Q}^{o T} \boldsymbol{\gamma}_{\alpha}^{o}\right) \otimes \boldsymbol{e}_{\alpha}^{r}+\boldsymbol{Q}^{o} \\
& =\boldsymbol{Q}^{o}\left(\boldsymbol{I}+\left(\boldsymbol{Q}^{o T} \boldsymbol{\gamma}_{\alpha}^{o}\right) \otimes \boldsymbol{e}_{\alpha}^{r}\right)=\boldsymbol{Q}^{o}\left(\boldsymbol{I}+\boldsymbol{\gamma}_{\alpha}^{o r} \otimes \boldsymbol{e}_{\alpha}^{r}\right)=\boldsymbol{Q}^{o} \boldsymbol{F}^{o r}
\end{aligned}
$$

O tensor $\boldsymbol{F}^{\text {or }}$ é chamado de gradiente da transformação inicial retro-rotacionado e pode-se obter seus vetores-coluna $f_{i}^{o r}$ fazendo-se

$$
\begin{aligned}
\boldsymbol{F}^{o r} & =\boldsymbol{I}+\boldsymbol{\gamma}_{\alpha}^{o r} \otimes \boldsymbol{e}_{\alpha}^{r}=\boldsymbol{e}_{i}^{r} \otimes \boldsymbol{e}_{i}^{r}+\boldsymbol{\gamma}_{\alpha}^{o r} \otimes \boldsymbol{e}_{\alpha}^{r} \\
& =\left(\boldsymbol{e}_{\alpha}^{r}+\gamma_{\alpha}^{o r}\right) \otimes \boldsymbol{e}_{\alpha}^{r}+\boldsymbol{e}_{3}^{r} \otimes \boldsymbol{e}_{3}^{r}=\boldsymbol{f}_{\alpha}^{o r} \otimes \boldsymbol{e}_{\alpha}^{r}+\boldsymbol{f}_{3}^{o r} \otimes \boldsymbol{e}_{3}^{r}, \\
& =\boldsymbol{f}_{i}^{o r} \otimes \boldsymbol{e}_{i}^{r}
\end{aligned}
$$

tal que

$$
\begin{aligned}
& \boldsymbol{f}_{\alpha}^{o r}=\boldsymbol{e}_{\alpha}^{r}+\gamma_{\alpha}^{o r} \\
& \boldsymbol{f}_{3}^{o r}=\boldsymbol{e}_{3}^{r}
\end{aligned}
$$


Será útil, também, o cálculo da inversa da transformação inicial $\boldsymbol{F}^{o}$. De (4.21), temos

$$
\begin{aligned}
\boldsymbol{F}^{o-1} & =\left(\boldsymbol{Q}^{o} \boldsymbol{F}^{o r}\right)^{-1}=\boldsymbol{F}^{o r-1} \boldsymbol{Q}^{o-1}=\boldsymbol{F}^{o r-1} \boldsymbol{Q}^{o T} \\
& =\frac{1}{J^{o}}\left(\boldsymbol{g}_{i}^{o r} \otimes \boldsymbol{e}_{i}^{r}\right)^{T} \boldsymbol{Q}^{o T}=\frac{1}{J^{o}}\left(\boldsymbol{e}_{i}^{r} \otimes \boldsymbol{g}_{i}^{o r}\right) \boldsymbol{Q}^{o T}
\end{aligned}
$$

onde

$$
\begin{aligned}
J^{o} & =\operatorname{det} \boldsymbol{F}^{o}=\boldsymbol{e}_{3}^{r} \cdot \boldsymbol{f}_{1}^{o r} \times \boldsymbol{f}_{2}^{o r} \\
\boldsymbol{g}_{1}^{o r} & =\boldsymbol{f}_{2}^{o r} \times \boldsymbol{e}_{3}^{r} \\
\boldsymbol{g}_{2}^{o r} & =\boldsymbol{e}_{3}^{r} \times \boldsymbol{f}_{1}^{o r} \\
\boldsymbol{g}_{3}^{o r} & =\boldsymbol{f}_{1}^{o r} \times \boldsymbol{f}_{2}^{o r}
\end{aligned}
$$

\subsubsection{Definição da Transformação Inicial}

A configuração inicial da casca pode ser descrita por meio de uma descrição analítica ou aproximada dos pontos da superfície de referência. Um exemplo de descrição aproximada seria descrever a posição desses pontos através de funções Moving Least Squares, de Elementos Finitos ou qualquer outro tipo de discretização que permita-nos obter a posição inicial $\boldsymbol{z}^{o}$ dos pontos e suas tangentes $\boldsymbol{z}_{, \alpha}^{o}$.

A forma analítica de fazê-lo se aplica a cascas cuja configuração inicial pode ser expressa facilmente por funções deriváveis:

\section{Placa Plana}

Para o mapeamento de placa plana, que leva à teoria apresentada no Capítulo 3, consideremos um domínio quadrado

$$
\begin{aligned}
& 0 \leq \xi_{1} \leq a \\
& 0 \leq \xi_{2} \leq b
\end{aligned}
$$


O mapeamento pode deformar uniformemente essas dimensões com parâmetros $a_{1}$ e $a_{2}$ de forma que a configuração inicial será

$$
\boldsymbol{z}^{o}=\left\{\begin{array}{c}
a_{1} \xi_{1} \\
a_{2} \xi_{2} \\
0
\end{array}\right\} .
$$

Essa transformação inicial gera uma casca como a ilustrada na Figura 19.

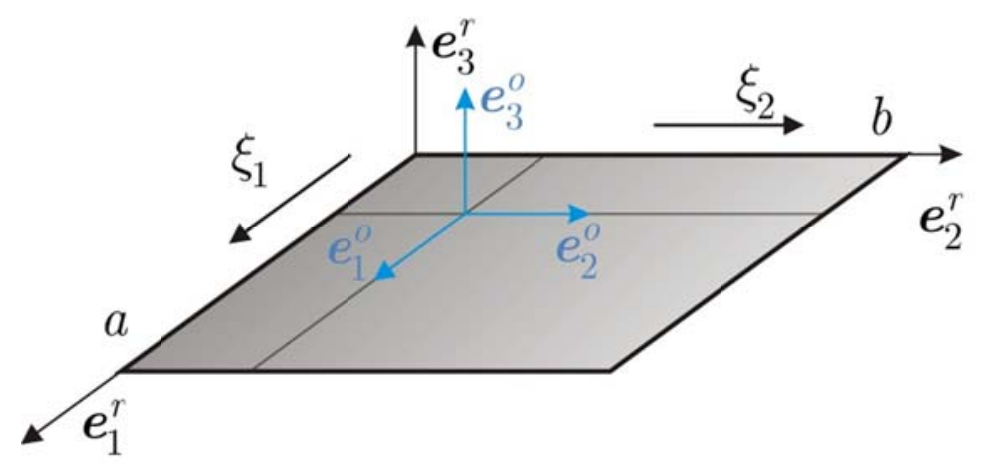

Figura 19 - Mapeamento inicial para placa plana

\section{Casca Cilíndrica}

Para o mapeamento cilíndrico, seja $\xi_{1}$ a coordenada ao longo do eixo do cilindro e $\xi_{2}$ a coordenada ao longo do comprimento do arco de circunferência.

$$
\begin{aligned}
& 0 \leq \xi_{1} \leq l \\
& 0 \leq \xi_{2} \leq 2 \pi r
\end{aligned}
$$

Definindo $k=\xi_{2} / r$, a configuração inicial será

$$
\boldsymbol{z}^{o}=\left\{\begin{array}{c}
\xi_{1} \\
r \cos (k) \\
r \sin (k)
\end{array}\right\}
$$


A Figura 20 ilustra esse mapeamento. Deve-se observar que $e_{3}^{o}$ tem direção radial e sentido para o centro do cilindro.

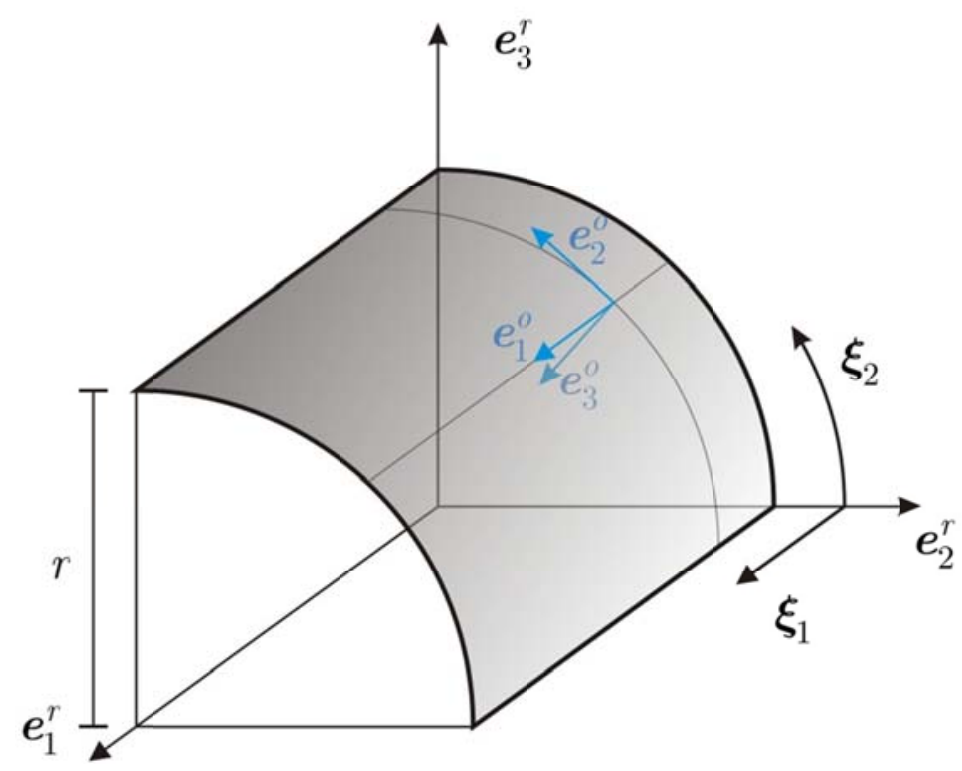

Figura 20 - Mapeamento inicial para casca cilíndrica

\section{Casca Hemisférica}

Para o mapeamento de casca hemisférica ilustrado na Figura $21, \xi_{1}$ e $\xi_{2}$ serão comprimentos de arco. Para a consideração de um oitavo de esfera, teremos um domínio quadrado

$$
\begin{aligned}
& 0 \leq \xi_{1} \leq \frac{\pi r}{2} \\
& 0 \leq \xi_{2} \leq \frac{\pi r}{2}
\end{aligned}
$$

que será mapeado para uma esfera com a ajuda de $\theta_{\alpha}=\xi_{\alpha} / r$. A configuração inicial será

$$
\boldsymbol{z}^{o}=\left\{\begin{array}{c}
r \sin \left(\theta_{2}\right) \cos \left(\theta_{1}\right) \\
r \sin \left(\theta_{2}\right) \sin \left(\theta_{1}\right) \\
r \cos \left(\theta_{2}\right)
\end{array}\right\}
$$




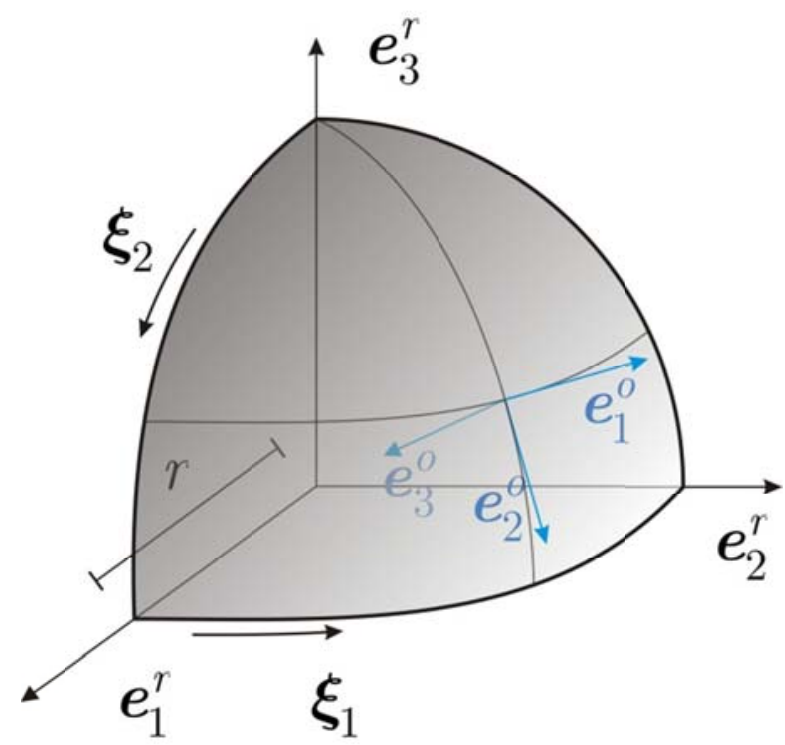

Figura 21 - Mapeamento inicial para casca esférica

\subsection{Transformação Efetiva}

A partir da configuração inicial é que se dará o movimento real (com tensões). A este, dá-se o nome de transformação efetiva. A posição final de um ponto da casca pode ser definida por

$$
x=z+a
$$

onde $z$ é a posição da superfície de referência e $\boldsymbol{a}$ é o diretor. A superfície de referência terá se deslocado $\boldsymbol{u}$ de sua posição na configuração inicial, ou seja,

$$
z=z^{o}+u
$$

e o diretor terá sido rotacionado, mais uma vez sem alterar sua magnitude. Expressando essa rotação efetiva por $\boldsymbol{Q}^{e}$, temos

$$
\boldsymbol{a}=\boldsymbol{Q}^{e} \boldsymbol{a}^{o}=\boldsymbol{Q \boldsymbol { a } ^ { r }}
$$

Por sua vez, $Q$ é a rotação total, da configuração de referência à atual, que, por composição, é 


$$
\boldsymbol{Q}=\boldsymbol{Q}^{e} \boldsymbol{Q}^{o}
$$

Ao contrario da rotação inicial, $\boldsymbol{Q}^{e}$ é aproximada independentemente do campo de deslocamentos $\boldsymbol{u}$, sendo parametrizada pelo vetor rotação $\boldsymbol{\theta}$, que recolhe os ditos parâmetros de Euler, representantes da rotação através de

$$
\boldsymbol{Q}^{e}=\boldsymbol{I}+h_{1}(\theta) \boldsymbol{\Theta}+h_{2}(\theta) \boldsymbol{\Theta}^{2}
$$

conhecida como fórmula de Euler-Rodrigues, onde

$$
\begin{aligned}
\boldsymbol{\Theta} & =\operatorname{Skew}(\boldsymbol{\theta})=\left[\begin{array}{ccc}
0 & -\theta_{3} & \theta_{2} \\
\theta_{3} & 0 & -\theta_{1} \\
-\theta_{2} & \theta_{1} & 0
\end{array}\right], \\
\theta & =\|\boldsymbol{\theta}\|, \\
h_{1}(\theta) & =\frac{\sin (\theta)}{\theta}, \\
h_{2}(\theta) & =\frac{1}{2}\left(\frac{\sin (\theta / 2)}{\theta / 2}\right)^{2}
\end{aligned}
$$

As componentes de $\boldsymbol{u}$ e $\boldsymbol{\theta}$ em um sistema cartesiano global definem os seis graus de liberdade do modelo.

O gradiente da transformação total será:

$$
\begin{aligned}
\boldsymbol{F} & =\frac{\partial \boldsymbol{x}}{\partial \boldsymbol{\xi}}=\frac{\partial \boldsymbol{x}}{\partial \xi_{\alpha}} \otimes \boldsymbol{e}_{\alpha}^{r}+\frac{\partial \boldsymbol{x}}{\partial \xi_{3}} \otimes \boldsymbol{e}_{3}^{r} \\
& =(\boldsymbol{z}+\boldsymbol{a})_{, \alpha} \otimes \boldsymbol{e}_{\alpha}^{r}+(\boldsymbol{z}+\boldsymbol{a})^{\prime} \otimes \boldsymbol{e}_{3}^{r} \\
& =\left(\boldsymbol{z}_{, \alpha}^{o}+\boldsymbol{u}_{, \alpha}+\boldsymbol{Q}_{, \alpha} \boldsymbol{Q}^{T} \boldsymbol{a}\right) \otimes \boldsymbol{e}_{\alpha}^{r}+\boldsymbol{e}_{3} \otimes \boldsymbol{e}_{3}^{r} \\
& =\left(\boldsymbol{z}_{, \alpha}^{o}+\boldsymbol{u}_{, \alpha}-\boldsymbol{e}_{\alpha}+\boldsymbol{Q}_{, \alpha} \boldsymbol{Q}^{T} \boldsymbol{a}\right) \otimes \boldsymbol{e}_{\alpha}^{r}+\boldsymbol{e}_{i} \otimes \boldsymbol{e}_{i}^{r} \\
& =\left(\boldsymbol{\eta}_{\alpha}+\boldsymbol{K}_{\alpha} \boldsymbol{a}\right) \otimes \boldsymbol{e}_{\alpha}^{r}+\boldsymbol{Q}
\end{aligned}
$$

onde, similarmente a (4.20), 


$$
\boldsymbol{\eta}_{\alpha}=\boldsymbol{z}_{, \alpha}^{o}+\boldsymbol{u}_{, \alpha}-\boldsymbol{e}_{\alpha}=\boldsymbol{z}_{, \alpha}-\boldsymbol{Q} \boldsymbol{e}_{\alpha}^{r}
$$

e os tensores $\boldsymbol{K}_{\alpha}$ podem ser expressos como

$$
\begin{aligned}
\boldsymbol{K}_{\alpha} & =\boldsymbol{Q}_{, \alpha} \boldsymbol{Q}^{T}=\left(\boldsymbol{Q}^{e} \boldsymbol{Q}^{o}\right)_{, \alpha}\left(\boldsymbol{Q}^{e} \boldsymbol{Q}^{o}\right)^{T}=\left(\boldsymbol{Q}_{, \alpha}^{e} \boldsymbol{Q}^{o}+\boldsymbol{Q}^{e} \boldsymbol{Q}_{, \alpha}^{o}\right) \boldsymbol{Q}^{o T} \boldsymbol{Q}^{e T} \\
& =\boldsymbol{Q}_{, \alpha}^{e} \boldsymbol{Q}^{o} \boldsymbol{Q}^{o T} \boldsymbol{Q}^{e T}+\boldsymbol{Q}^{e} \boldsymbol{Q}_{, \alpha}^{o} \boldsymbol{Q}^{o T} \boldsymbol{Q}^{e T}=\boldsymbol{Q}_{, \alpha}^{e} \boldsymbol{Q}^{e T}+\boldsymbol{Q}^{e} \boldsymbol{K}_{\alpha}^{o} \boldsymbol{Q}^{e T} \\
& =\boldsymbol{K}_{\alpha}^{e}+\boldsymbol{Q}^{e} \boldsymbol{K}_{\alpha}^{o} \boldsymbol{Q}^{e T}
\end{aligned}
$$

expressão na qual

$$
\begin{aligned}
\boldsymbol{K}_{\alpha}^{o} & =\boldsymbol{Q}_{, \alpha}^{o} \boldsymbol{Q}^{o T} \\
\boldsymbol{K}_{\alpha}^{e} & =\boldsymbol{Q}_{, \alpha}^{e} \boldsymbol{Q}^{e T}
\end{aligned}
$$

podem ser identificados respectivamente com as deformações iniciais e efetivas. Os vetores axiais de $\boldsymbol{K}_{\alpha}$ são dados por

$$
\boldsymbol{\kappa}_{\alpha}=\operatorname{axial}\left(\boldsymbol{K}_{\alpha}^{e}+\boldsymbol{Q}^{e} \boldsymbol{K}_{\alpha}^{e} \boldsymbol{Q}^{e T}\right)=\boldsymbol{\kappa}_{\alpha}^{e}+\boldsymbol{Q}^{e} \boldsymbol{\kappa}_{\alpha}^{o}
$$

onde $\kappa_{\alpha}^{o}$ é dado por (4.15),

$$
\boldsymbol{\kappa}_{\alpha}^{e}=\operatorname{axial}\left(\boldsymbol{K}_{\alpha}^{e}\right)=\boldsymbol{\Gamma} \boldsymbol{\theta}_{, \alpha}
$$

e

$$
\begin{aligned}
& \boldsymbol{\Gamma}=I+h_{2}(\theta) \boldsymbol{\Theta}+h_{3}(\theta) \boldsymbol{\Theta}^{2} \\
& h_{3}(\theta)=\frac{1-h_{1}(\theta)}{\theta^{2}}
\end{aligned}
$$

Similarmente ao desenvolvido para as deformações iniciais, e com o mesmo objetivo de obterem-se definições objetivas, deve se retro-rotacionar as deformações generalizadas 


$$
\boldsymbol{\eta}_{\alpha}^{r}=\boldsymbol{Q}^{T} \boldsymbol{\eta}_{\alpha}=\boldsymbol{Q}^{T} \boldsymbol{z}_{, \alpha}-\boldsymbol{e}_{\alpha}^{r}
$$

e

$$
\begin{aligned}
\boldsymbol{\kappa}_{\alpha}^{r} & =\boldsymbol{Q}^{T} \boldsymbol{\kappa}_{\alpha}=\boldsymbol{Q}^{T}\left(\boldsymbol{\kappa}_{\alpha}^{e}+\boldsymbol{Q}^{e} \boldsymbol{\kappa}_{\alpha}^{o}\right)=\boldsymbol{\kappa}_{\alpha}^{e r}+\boldsymbol{Q}^{T} \boldsymbol{Q} \boldsymbol{Q}^{o T} \boldsymbol{\kappa}_{\alpha}^{o} \\
& =\boldsymbol{\kappa}_{\alpha}^{e r}+\boldsymbol{Q}^{o T} \boldsymbol{\kappa}_{\alpha}^{o}=\boldsymbol{\kappa}_{\alpha}^{e r}+\boldsymbol{\kappa}_{\alpha}^{o r}
\end{aligned}
$$

que também podem ser expressos como

$$
\begin{aligned}
\boldsymbol{\kappa}_{\alpha}^{r} & =\boldsymbol{Q}^{T} \boldsymbol{\kappa}_{\alpha}=\boldsymbol{Q}^{T}\left(\boldsymbol{\kappa}_{\alpha}^{e}+\boldsymbol{Q}^{e} \boldsymbol{\kappa}_{\alpha}^{o}\right)=\boldsymbol{Q}^{T}\left(\boldsymbol{\Gamma} \boldsymbol{\theta}_{, \alpha}+\boldsymbol{Q}^{e} \boldsymbol{\kappa}_{\alpha}^{o}\right) \\
& =\boldsymbol{Q}^{T}\left(\boldsymbol{Q}^{e} \boldsymbol{\Gamma}^{T} \boldsymbol{\theta}_{, \alpha}+\boldsymbol{Q}^{e} \boldsymbol{\kappa}_{\alpha}^{o}\right)=\boldsymbol{Q}^{T} \boldsymbol{Q}^{e}\left(\boldsymbol{\Gamma}^{T} \boldsymbol{\theta}_{, \alpha}+\boldsymbol{\kappa}_{\alpha}^{o}\right) \\
& =\boldsymbol{Q}^{o T}\left(\boldsymbol{\Gamma}^{T} \boldsymbol{\theta}_{, \alpha}+\boldsymbol{\kappa}_{\alpha}^{o}\right)
\end{aligned}
$$

Com essas definições, o gradiente da transformação total dado por (4.38) pode ser expresso por

$$
\begin{aligned}
\boldsymbol{F} & =\left(\boldsymbol{\eta}_{\alpha}+\boldsymbol{K}_{\alpha} \boldsymbol{a}\right) \otimes \boldsymbol{e}_{\alpha}^{r}+\boldsymbol{Q}=\boldsymbol{Q}\left(\boldsymbol{Q}^{T}\left(\boldsymbol{\eta}_{\alpha}+\boldsymbol{K}_{\alpha} \boldsymbol{a}\right) \otimes \boldsymbol{e}_{\alpha}^{r}+\boldsymbol{I}\right) \\
& =\boldsymbol{Q}\left(\boldsymbol{I}+\left(\boldsymbol{\eta}_{\alpha}^{r}+\boldsymbol{Q}^{T}\left(\boldsymbol{\kappa}_{\alpha} \times \boldsymbol{a}\right)\right) \otimes \boldsymbol{e}_{\alpha}^{r}\right) \\
& =\boldsymbol{Q}\left(\boldsymbol{I}+\left(\boldsymbol{\eta}_{\alpha}^{r}+\left(\boldsymbol{\kappa}_{\alpha}^{r} \times \boldsymbol{a}\right)\right) \otimes \boldsymbol{e}_{\alpha}^{r}\right)=\boldsymbol{Q}\left(\boldsymbol{I}+\boldsymbol{\gamma}_{\alpha}^{r} \otimes \boldsymbol{e}_{\alpha}^{r}\right)
\end{aligned}
$$

onde surgiram os vetores das deformações totais generalizadas retro-rotacionadas

$$
\boldsymbol{\gamma}_{\alpha}^{r}=\boldsymbol{\eta}_{\alpha}^{r}+\boldsymbol{\kappa}_{\alpha}^{r} \times \boldsymbol{a}^{r}
$$

De (4.48) também pode se retirar outra representação para $\boldsymbol{F}$

$$
\boldsymbol{F}=\boldsymbol{Q} \boldsymbol{F}^{r}
$$

com 


$$
\begin{aligned}
\boldsymbol{F}^{r} & =\boldsymbol{I}+\gamma_{\alpha}^{r} \otimes \boldsymbol{e}_{\alpha}^{r}=\boldsymbol{e}_{i}^{r} \otimes \boldsymbol{e}_{i}^{r}+\gamma_{\alpha}^{r} \otimes \boldsymbol{e}_{\alpha}^{r} \\
& =\left(\boldsymbol{e}_{\alpha}^{r}+\gamma_{\alpha}^{r}\right) \otimes \boldsymbol{e}_{\alpha}^{r}+\boldsymbol{e}_{3}^{r} \otimes \boldsymbol{e}_{3}^{r}=\boldsymbol{f}_{i}^{r} \otimes \boldsymbol{e}_{i}^{r},
\end{aligned}
$$

o gradiente da transformação total retro-rotacionado separado em seus vetores-coluna

$$
\begin{aligned}
& \boldsymbol{f}_{\alpha}^{r}=\boldsymbol{e}_{\alpha}^{r}+\boldsymbol{\gamma}_{\alpha}^{r}=\boldsymbol{Q}^{T} \boldsymbol{z}_{, \alpha}+\left(\boldsymbol{\kappa}_{\alpha}^{\mathrm{e} r}+\boldsymbol{\kappa}_{\alpha}^{\mathrm{o} r}\right) \times \boldsymbol{a}^{r} \\
& \boldsymbol{f}_{3}^{r}=\boldsymbol{e}_{3}^{r}
\end{aligned}
$$

O gradiente da velocidade $\dot{\boldsymbol{F}}$ pode ser obtido por diferenciação de (4.50):

$$
\begin{aligned}
\dot{\boldsymbol{F}} & =\dot{\boldsymbol{Q}} \boldsymbol{F}^{r}+\boldsymbol{Q} \dot{\boldsymbol{F}}^{r}=\dot{\boldsymbol{Q}}\left(\boldsymbol{I}+\boldsymbol{\gamma}_{\alpha}^{r} \otimes \boldsymbol{e}_{\alpha}^{r}\right)+\boldsymbol{Q} \overline{\left(\boldsymbol{I}+\boldsymbol{\gamma}_{\alpha}^{r} \otimes \boldsymbol{e}_{\alpha}^{r}\right)} \\
& =\boldsymbol{\Omega} \boldsymbol{Q}\left(\boldsymbol{I}+\boldsymbol{\gamma}_{\alpha}^{r} \otimes \boldsymbol{e}_{\alpha}^{r}\right)+\boldsymbol{Q}\left(\dot{\boldsymbol{I}}+\dot{\gamma}_{\alpha}^{r} \otimes \boldsymbol{e}_{\alpha}^{r}+\gamma_{\alpha}^{r} \otimes \dot{\boldsymbol{e}}_{\alpha}^{r}\right) \\
& =\boldsymbol{\Omega} \boldsymbol{F}+\boldsymbol{Q}\left(\dot{\boldsymbol{\gamma}}_{\alpha}^{r} \otimes \boldsymbol{e}_{\alpha}^{r}\right)
\end{aligned}
$$

sendo $\boldsymbol{\Omega}=\dot{\boldsymbol{Q}} \boldsymbol{Q}^{T}$ o tensor de spin associado à rotação do diretor e $\dot{\gamma}_{\alpha}^{r}$ obtido de (4.49)

$$
\dot{\boldsymbol{\gamma}}_{\alpha}^{r}=\dot{\boldsymbol{\eta}}_{\alpha}^{r}+\dot{\boldsymbol{\kappa}}_{\alpha}^{r} \times \boldsymbol{a}^{r}
$$

com

$$
\dot{\boldsymbol{\eta}}_{\alpha}^{r}=\boldsymbol{Q}^{T}\left(\dot{\boldsymbol{u}}_{, \alpha}+\boldsymbol{Z}_{, \alpha} \boldsymbol{\Gamma} \dot{\boldsymbol{\theta}}\right) \quad \text { e } \quad \dot{\boldsymbol{\kappa}}_{\alpha}^{r}=\boldsymbol{Q}^{T}\left(\boldsymbol{\Gamma}_{, \alpha} \dot{\boldsymbol{\theta}}+\boldsymbol{\Gamma} \dot{\boldsymbol{\theta}}_{, \alpha}\right)
$$

onde foram usadas as definições de

$$
\boldsymbol{Z}_{, \alpha}=\operatorname{Skew}\left(\boldsymbol{z}_{, \alpha}\right)
$$

$\mathrm{e}$

$$
\begin{array}{r}
\boldsymbol{\Gamma}_{, \alpha}=h_{2}(\theta) \boldsymbol{\Theta}_{, \alpha}+h_{3}(\theta)\left(\boldsymbol{\Theta}_{, \alpha}+\boldsymbol{\Theta}_{, \alpha} \boldsymbol{\Theta}\right)+ \\
+h_{4}(\theta)\left(\boldsymbol{\theta} \cdot \boldsymbol{\theta}_{, \alpha}\right) \boldsymbol{\Theta}+h_{5}(\theta)\left(\boldsymbol{\theta} \cdot \boldsymbol{\theta}_{, \alpha}\right) \boldsymbol{\Theta}^{2}
\end{array}
$$




$$
h_{4}(\theta)=\frac{h_{1}(\theta)-2 h_{2}(\theta)}{\theta^{2}} \quad \text { e } \quad h_{5}(\theta)=\frac{h_{2}(\theta)-3 h_{3}(\theta)}{\theta^{2}}
$$

Através da composição de transformações $\boldsymbol{F}=\boldsymbol{F}^{e} \boldsymbol{F}^{o}$, pode-se definir também o gradiente da transformação efetiva $\boldsymbol{F}^{e}=\boldsymbol{F F}^{o-1}$, sendo $\boldsymbol{F}^{o-1}$ obtido por (4.24).

$$
\begin{aligned}
\boldsymbol{F}^{e} & =\boldsymbol{F} \boldsymbol{F}^{o-1}=\boldsymbol{Q}\left(\boldsymbol{f}_{i}^{r} \otimes \boldsymbol{e}_{i}^{r}\right) J^{o-1}\left(\boldsymbol{e}_{j}^{r} \otimes \boldsymbol{g}_{j}^{o r}\right) \boldsymbol{Q}^{o T} \\
& =\boldsymbol{Q} J^{o-1}\left(\boldsymbol{f}_{i}^{r} \otimes \boldsymbol{g}_{j}^{o r}\right) \delta_{i j} \boldsymbol{Q}^{o T}=\boldsymbol{Q J J}^{o-1}\left(\boldsymbol{f}_{i}^{r} \otimes \boldsymbol{g}_{i}^{o r}\right) \boldsymbol{Q}^{o T} \\
& =\boldsymbol{Q} J^{o-1}\left(\boldsymbol{f}_{i}^{r} \otimes \boldsymbol{g}_{i}^{o r}\right)\left(\boldsymbol{e}_{j}^{r} \otimes \boldsymbol{e}_{j}^{r}\right) \boldsymbol{Q}^{o T} \\
& =\boldsymbol{Q} J^{o-1}\left(\boldsymbol{f}_{i}^{r} \otimes \boldsymbol{e}_{j}^{r}\right)\left(\boldsymbol{g}_{i}^{o r} \cdot \boldsymbol{e}_{j}^{r}\right) \boldsymbol{Q}^{o T} \\
& =\boldsymbol{Q}\left(J^{o-1} \boldsymbol{f}_{i}^{r}\left(\boldsymbol{g}_{i}^{o r} \cdot \boldsymbol{e}_{j}^{r}\right) \otimes \boldsymbol{e}_{j}^{r}\right) \boldsymbol{Q}^{o T} \\
& =\boldsymbol{Q}\left(\boldsymbol{f}_{i}^{e r} \otimes \boldsymbol{e}_{i}^{r}\right) \boldsymbol{Q}^{o T}=\boldsymbol{Q}\left(\boldsymbol{f}_{i}^{e r} \otimes\left(\boldsymbol{Q}^{o} \boldsymbol{e}_{i}^{r}\right)\right) \\
& =\boldsymbol{Q}\left(\boldsymbol{f}_{i}^{e r} \otimes \boldsymbol{e}_{i}^{o}\right)
\end{aligned}
$$

Levando-se em consideração que, a partir de (4.25), $\boldsymbol{g}_{3}^{o r} \cdot \boldsymbol{e}_{\alpha}^{r}=0$, pode-se chegar a

$$
\begin{aligned}
& \boldsymbol{f}_{\alpha}^{e r}=J^{o-1}\left(\boldsymbol{e}_{\alpha}^{r} \cdot \boldsymbol{g}_{\beta}^{o r}\right) \boldsymbol{f}_{\beta}^{r} \\
& \boldsymbol{f}_{3}^{e r}=\boldsymbol{f}_{3}^{r}=\boldsymbol{e}_{3}^{r}
\end{aligned}
$$

Por analogia, definem-se as deformações efetivas retro-rotacionadas como

$$
\boldsymbol{\gamma}_{\alpha}^{e r}=\boldsymbol{f}_{\alpha}^{e r}-\boldsymbol{e}_{\alpha}^{r}=J^{o-1}\left(\boldsymbol{e}_{\alpha}^{r} \cdot \boldsymbol{g}_{\beta}^{o r}\right)\left(\boldsymbol{e}_{\beta}^{r}+\boldsymbol{\gamma}_{\beta}^{r}\right)-\boldsymbol{e}_{\alpha}^{r}
$$

\section{$4.3 \quad$ Estática}

O primeiro tensor de Piola-Kirchhoff efetivo é

$$
\boldsymbol{P}^{e}=J^{e} \boldsymbol{T} \boldsymbol{F}^{e-T}
$$

onde $J^{e}=\operatorname{det} \boldsymbol{F}^{e}$ é o Jacobiano da transformação efetiva e $\boldsymbol{T}$ é o tensor das tensões de Cauchy. Os vetores colunas de $\boldsymbol{P}^{e}$ são os vetores reais de tensão atuando em um ponto da 
casca na configuração atual, por unidade de área da configuração inicial. Seu correspondente "total" pode ser definido por

$$
\boldsymbol{P}=J \boldsymbol{T} \boldsymbol{F}^{-T}=J^{o} J^{e} \boldsymbol{T} \boldsymbol{F}^{e-T} \boldsymbol{F}^{o-T}=J^{o} \boldsymbol{P}^{e} \boldsymbol{F}^{o-T}
$$

Separando (4.62) e (4.63) em termo de seus vetores-coluna, obtemos

$$
\begin{aligned}
\boldsymbol{P}^{e} & =\boldsymbol{\tau}_{i}^{e} \otimes \boldsymbol{e}_{i}^{o}=\boldsymbol{Q} \boldsymbol{\tau}_{i}^{e r} \otimes \boldsymbol{e}_{i}^{o} \\
\boldsymbol{P} & =\boldsymbol{\tau}_{i} \otimes \boldsymbol{e}_{i}^{r}=\boldsymbol{Q} \boldsymbol{\tau}_{i}^{r} \otimes \boldsymbol{e}_{i}^{r}
\end{aligned}
$$

Aplicando (4.24) em (4.63), pode se chegar a

$$
\begin{aligned}
\boldsymbol{P} & =J^{o} \boldsymbol{P}^{e} \boldsymbol{F}^{o-T}=J^{o} \boldsymbol{P}^{e}\left(J^{o-1}\left(\boldsymbol{e}_{i}^{r} \otimes \boldsymbol{g}_{i}^{o r}\right) \boldsymbol{Q}^{o T}\right)^{T} \\
& =\boldsymbol{P}^{e} \boldsymbol{Q}^{o}\left(\boldsymbol{g}_{i}^{o r} \otimes \boldsymbol{e}_{i}^{r}\right)=\left(\boldsymbol{\tau}_{i}^{e} \otimes \boldsymbol{e}_{i}^{o}\right) \boldsymbol{Q}^{o}\left(\boldsymbol{g}_{i}^{o r} \otimes \boldsymbol{e}_{i}^{r}\right) \\
& =\left(\boldsymbol{Q} \boldsymbol{\tau}_{i}^{e r} \otimes \boldsymbol{e}_{i}^{o}\right) \boldsymbol{Q}^{o}\left(\boldsymbol{g}_{i}^{o r} \otimes \boldsymbol{e}_{i}^{r}\right)=\left(\boldsymbol{Q} \boldsymbol{\tau}_{i}^{e r} \otimes \boldsymbol{e}_{i}^{r}\right)\left(\boldsymbol{g}_{i}^{o r} \otimes \boldsymbol{e}_{i}^{r}\right) \\
& =\left(\boldsymbol{Q} \boldsymbol{\tau}_{i}^{e r} \otimes \boldsymbol{e}_{j}^{r}\right)\left(\boldsymbol{g}_{j}^{o r} \cdot \boldsymbol{e}_{i}^{r}\right)=\boldsymbol{Q}\left(\boldsymbol{g}_{j}^{o r} \cdot \boldsymbol{e}_{i}^{r}\right) \boldsymbol{\tau}_{i}^{e r} \otimes \boldsymbol{e}_{j}^{r}=\boldsymbol{Q} \boldsymbol{\tau}_{i}^{r} \otimes \boldsymbol{e}_{i}^{r}
\end{aligned}
$$

onde $\boldsymbol{\tau}_{i}^{r}=\left(\boldsymbol{g}_{j}^{o r} \cdot \boldsymbol{e}_{i}^{r}\right) \boldsymbol{\tau}_{i}^{e r}$ são as tensões retro-rotacionadas para a configuração de referência, também dadas por

$$
\begin{aligned}
\boldsymbol{\tau}_{\alpha}^{r} & =\left(\boldsymbol{g}_{\alpha}^{o r} \cdot \boldsymbol{e}_{\beta}^{r}\right) \boldsymbol{\tau}_{\beta}^{e r} \\
\boldsymbol{\tau}_{3}^{r} & =\left(\boldsymbol{g}_{\alpha}^{o r} \cdot \boldsymbol{e}_{3}^{r}\right) \boldsymbol{\tau}_{3}^{e r}=\left(\boldsymbol{f}_{1}^{o r} \cdot \boldsymbol{f}_{2}^{o r} \times \boldsymbol{f}_{3}^{o r}\right) \boldsymbol{\tau}_{3}^{e r}=J^{o} \boldsymbol{\tau}_{3}^{e r}
\end{aligned}
$$

A potência interna por volume unitário na configuração de referência pode ser expressa por

$$
\boldsymbol{P}: \dot{\boldsymbol{F}}=J^{o} \boldsymbol{P}^{e}: \dot{\boldsymbol{F}}^{e}
$$

de forma que a integração dessa potência pode ser feita na configuração de referência (plana). Explorando a simetria de $\boldsymbol{P} \boldsymbol{F}^{T}$ e a ortogonalidade de $\boldsymbol{Q}$, 


$$
\begin{aligned}
\boldsymbol{P}: \dot{\boldsymbol{F}} & =\boldsymbol{P}:\left(\boldsymbol{\Omega} \boldsymbol{F}+\boldsymbol{Q}\left(\dot{\boldsymbol{\gamma}}_{\alpha}^{r} \otimes \boldsymbol{e}_{\alpha}^{r}\right)\right)=\boldsymbol{P}: \boldsymbol{\Omega} \boldsymbol{F}+\boldsymbol{P}: \boldsymbol{Q}\left(\dot{\boldsymbol{\gamma}}_{\alpha}^{r} \otimes \boldsymbol{e}_{\alpha}^{r}\right) \\
& =\boldsymbol{P} \boldsymbol{F}^{T}: \boldsymbol{\Omega}+\left(\boldsymbol{Q} \boldsymbol{\tau}_{i}^{r} \otimes \boldsymbol{e}_{i}^{r}\right): \boldsymbol{Q}\left(\dot{\boldsymbol{\gamma}}_{\alpha}^{r} \otimes \boldsymbol{e}_{\alpha}^{r}\right)=\boldsymbol{Q}\left(\boldsymbol{\tau}_{i}^{r} \otimes \boldsymbol{e}_{i}^{r}\right): \boldsymbol{Q}\left(\dot{\boldsymbol{\gamma}}_{\alpha}^{r} \otimes \boldsymbol{e}_{\alpha}^{r}\right) \\
& =\left(\boldsymbol{\tau}_{i}^{r} \otimes \boldsymbol{e}_{i}^{r}\right):\left(\dot{\boldsymbol{\gamma}}_{\alpha}^{r} \otimes \boldsymbol{e}_{\alpha}^{r}\right)=\boldsymbol{\tau}_{\alpha}^{r} \cdot \dot{\boldsymbol{\gamma}}_{\alpha}^{r}=\boldsymbol{\tau}_{\alpha}^{r} \cdot\left(\dot{\boldsymbol{\eta}}_{\alpha}^{r}+\dot{\boldsymbol{\kappa}}_{\alpha}^{r} \times \boldsymbol{a}^{r}\right) \\
& =\boldsymbol{\tau}_{\alpha}^{r} \cdot \dot{\boldsymbol{\eta}}_{\alpha}^{r}+\boldsymbol{\tau}_{\alpha}^{r} \cdot\left(\dot{\boldsymbol{\kappa}}_{\alpha}^{r} \times \boldsymbol{a}^{r}\right) \\
& =\boldsymbol{\tau}_{\alpha}^{r} \cdot \dot{\boldsymbol{\eta}}_{\alpha}^{r}+\left(\boldsymbol{a}^{r} \times \boldsymbol{\tau}_{\alpha}^{r}\right) \cdot \dot{\boldsymbol{\kappa}}_{\alpha}^{r}
\end{aligned}
$$

Deve-se notar que $\tau_{3}^{r}$ não executa trabalho, como se deve esperar de uma teoria em que não se considera deformação do diretor.

Considerando que $\dot{\boldsymbol{\eta}}_{\alpha}^{r}$ e $\dot{\boldsymbol{\kappa}}_{\alpha}^{r}$ são deformações generalizadas, que não variam na espessura, a integração de (4.68) na mesma gera

$$
\begin{aligned}
\int_{H}(\boldsymbol{P}: \dot{\boldsymbol{F}}) d H & =\int_{H}\left(\boldsymbol{\tau}_{\alpha}^{r} \cdot \dot{\boldsymbol{\eta}}_{\alpha}^{r}+\left(\boldsymbol{a}^{r} \times \boldsymbol{\tau}_{\alpha}^{r}\right) \cdot \dot{\boldsymbol{\kappa}}_{\alpha}^{r}\right) d H \\
& =\int_{H}\left(\boldsymbol{\tau}_{\alpha}^{r}\right) d H \cdot \dot{\boldsymbol{\eta}}_{\alpha}^{r}+\int_{H}\left(\boldsymbol{a}^{r} \times \boldsymbol{\tau}_{\alpha}^{r}\right) d H \cdot \dot{\boldsymbol{\kappa}}_{\alpha}^{r}, \\
& =\boldsymbol{n}_{\alpha}^{r} \cdot \dot{\boldsymbol{\eta}}_{\alpha}^{r}+\boldsymbol{m}_{\alpha}^{r} \cdot \dot{\boldsymbol{\kappa}}_{\alpha}^{r}
\end{aligned}
$$

onde foram definidos os vetores

$$
\boldsymbol{n}_{\alpha}^{r}=\int_{H}\left(\boldsymbol{\tau}_{\alpha}^{r}\right) d H \quad \text { e } \quad \boldsymbol{m}_{\alpha}^{r}=\int_{H}\left(\boldsymbol{a}^{r} \times \boldsymbol{\tau}_{\alpha}^{r}\right) d H
$$

que podem ser tomados como resultantes generalizadas dos esforços na seção transversal, sendo $\boldsymbol{n}_{\alpha}^{r}$ as resultantes de forças retro-rotacionadas e $\boldsymbol{m}_{\alpha}^{r}$ resultantes de momento, ambas por unidade de comprimento da configuração de referência. Essas grandezas não são afetadas por movimentos de corpo rígido.

Reunindo as resultantes da seção transversal em 


$$
\begin{aligned}
\boldsymbol{\sigma}_{\alpha}^{r} & =\left[\begin{array}{c}
\boldsymbol{n}_{\alpha}^{r} \\
\boldsymbol{m}_{\alpha}^{r}
\end{array}\right] \\
\boldsymbol{\varepsilon}_{\alpha}^{r} & =\left[\begin{array}{l}
\boldsymbol{\eta}_{\alpha}^{r} \\
\boldsymbol{\kappa}_{\alpha}^{r}
\end{array}\right], \\
\boldsymbol{d} & =\left[\begin{array}{l}
\boldsymbol{u} \\
\boldsymbol{\theta}
\end{array}\right]
\end{aligned}
$$

a potência por unidade de área, dada por (4.69), pode ser expressa por

$$
\int_{H}(\boldsymbol{P}: \dot{\boldsymbol{F}}) d H=\boldsymbol{\sigma}_{\alpha}^{r} \cdot \dot{\boldsymbol{\varepsilon}}_{\alpha}^{r}
$$

Ainda mais, as derivadas temporais das deformações generalizadas dadas por (4.55) podem ser compactamente escritas como

$$
\dot{\varepsilon}_{\alpha}^{r}=\Psi_{\alpha} \Delta_{\alpha} \dot{d}
$$

com o auxílio de

$$
\boldsymbol{\Psi}_{\alpha}=\left[\begin{array}{cc}
\boldsymbol{Q}^{T} & \boldsymbol{O} \\
\boldsymbol{O} & \boldsymbol{Q}^{T}
\end{array}\right]\left[\begin{array}{ccc}
\boldsymbol{I} & \boldsymbol{O} & \boldsymbol{Z}_{, \alpha} \boldsymbol{\Gamma} \\
\boldsymbol{O} & \boldsymbol{\Gamma} & \boldsymbol{\Gamma}_{, \alpha}
\end{array}\right], \quad \boldsymbol{\Delta}_{\alpha}=\left[\begin{array}{cc}
\boldsymbol{I} \frac{\partial}{\partial \xi_{\alpha}} & \boldsymbol{O} \\
\boldsymbol{O} & \boldsymbol{I} \frac{\partial}{\partial \xi_{\alpha}} \\
\boldsymbol{O} & \boldsymbol{I}
\end{array}\right] .
$$

A potência dos esforços internos em uma região delimitada da configuração de referência $\Omega^{r}$ será a integral de (4.67) no volume dessa região, ou de (4.69) na área da superfície de referência

$$
P_{\text {int }}=\int_{V^{r}}(\boldsymbol{P}: \dot{\boldsymbol{F}}) d V^{r}=\int_{\Omega^{r}}\left(\boldsymbol{\sigma}_{\alpha}^{r} \cdot \dot{\boldsymbol{\varepsilon}}_{\alpha}^{r}\right) d \Omega^{r}
$$

ao passo que a potência dos esforços externos será dada por 


$$
\begin{aligned}
P_{\mathrm{ext}} & =\int_{\Omega^{o}}\left[\overline{\boldsymbol{t}}^{o t} \cdot \dot{\boldsymbol{x}}^{t}+\overline{\boldsymbol{t}}^{o b} \cdot \dot{\boldsymbol{x}}^{b}+\int_{H^{o}} \overline{\boldsymbol{b}}^{o} \cdot \dot{\boldsymbol{x}} d H\right] d \Omega^{o} \\
& +\int_{\Gamma_{t}^{o}} \int_{H^{o}} \overline{\boldsymbol{t}}^{o l} \cdot \dot{\boldsymbol{x}} d H^{o} d \Gamma_{t}^{o}+\int_{\Gamma_{u}^{o}} \int_{H^{o}} \boldsymbol{r}^{o} \cdot \dot{\boldsymbol{x}} d H^{o} d \Gamma_{u}^{o} \\
P_{\mathrm{ext}} & =\int_{\Omega^{r}}\left[\overline{\boldsymbol{t}}^{t} \cdot \dot{\boldsymbol{x}}^{t}+\overline{\boldsymbol{t}}^{b} \cdot \dot{\boldsymbol{x}}^{b}+\int_{H^{r}} \overline{\boldsymbol{b}} \cdot \dot{\boldsymbol{x}} d H\right] d \Omega^{r} \\
& +\int_{\Gamma_{t}^{r}} \int_{H^{r}} \overline{\boldsymbol{t}}^{l} \cdot \dot{\boldsymbol{x}} d H^{r} d \Gamma_{t}^{r}+\int_{\Gamma_{u}^{r}} \int_{H^{r}} \boldsymbol{r}^{r} \cdot \dot{\boldsymbol{x}} d H^{r} d \Gamma_{u}^{r}
\end{aligned}
$$

onde $\overline{\boldsymbol{t}}^{o t}=J^{o t} \overline{\boldsymbol{t}}^{t}$ são respectivamente os esforços externos impostos no topo da casca por unidade de área na configuração inicial e de referência, sendo $J^{\text {ot }}$ o Jacobiano da transformação inicial avaliado no topo da casca. Similarmente, no fundo da casca, $\overline{\boldsymbol{t}}^{o b}=J^{o b} \overline{\boldsymbol{t}}^{b}$ e na lateral $\overline{\boldsymbol{t}}^{o l}=J^{o l} \overline{\boldsymbol{t}}^{l}$. Também, $\overline{\boldsymbol{b}}^{o}=J^{o} \overline{\boldsymbol{b}}$, que é a força de volume atuante na casca nas configurações iniciais e de referência, respectivamente. A integração se mantém já que os intervalos infinitesimais nas duas configurações se relacionam de forma similar às tensões, por exemplo, $d \Omega^{o}=J^{o} d \Omega^{r}$.

Deve-se notar também a presença das reações de apoio como forças externas. Isso é necessário em Método de Galerkin Livre de Elementos, pois não se pode impor, a priori, as condições de contorno essenciais. Desta forma, o espaço de teste (trial space) não satisfaz $\delta \boldsymbol{d}=\boldsymbol{O}$ em $\Gamma_{u}^{r}$, como é usual no método dos elementos finitos.

Usando $\dot{\boldsymbol{x}}=\dot{\boldsymbol{u}}+(\boldsymbol{\Gamma} \dot{\boldsymbol{\theta}}) \times \boldsymbol{a}=\dot{\boldsymbol{u}}+\boldsymbol{\omega} \times \boldsymbol{a}$,

$$
\begin{aligned}
P_{\mathrm{ext}} & =\int_{\Omega^{r}}\left(\overline{\boldsymbol{n}}^{\Omega^{r}} \cdot \dot{\boldsymbol{u}}+\overline{\boldsymbol{m}}^{\Omega^{r}} \cdot \boldsymbol{\omega}\right) d \Omega^{r}+\int_{\Gamma_{t}^{r}}\left(\overline{\boldsymbol{n}}^{\Gamma_{t}^{r}} \cdot \dot{\boldsymbol{u}}+\overline{\boldsymbol{m}}^{\Gamma_{t}^{r}} \cdot \boldsymbol{\omega}\right) d \Gamma_{t}^{r} \\
& +\int_{\Gamma_{u}^{r}}\left(\boldsymbol{n}^{\Gamma_{u}^{r}} \cdot \dot{\boldsymbol{u}}+\boldsymbol{m}^{\Gamma_{u}^{r}} \cdot \boldsymbol{\omega}\right) d \Gamma_{u}^{r}
\end{aligned}
$$

onde

$$
\begin{aligned}
& \overline{\boldsymbol{n}}^{\Omega^{r}}=\overline{\boldsymbol{t}}^{t r}+\overline{\boldsymbol{t}}^{b r}+\int_{H^{r}} \overline{\boldsymbol{b}}^{r} d H^{r} \\
& \overline{\boldsymbol{m}}^{\Omega^{r}}=\boldsymbol{a}^{t} \times \overline{\boldsymbol{t}}^{t r}+\boldsymbol{a}^{b} \times \overline{\boldsymbol{t}}^{b r}+\int_{H^{r}} \boldsymbol{a} \times \overline{\boldsymbol{b}}^{r} d H^{r}
\end{aligned}
$$

são respectivamente as resultantes de forças e os momentos generalizados impostos no domínio, 


$$
\begin{aligned}
& \overline{\boldsymbol{n}}^{\Gamma_{t}^{r}}=\int_{H^{r}} \overline{\boldsymbol{t}}^{l r} d H^{r} \\
& \overline{\boldsymbol{m}}^{\Gamma_{t}^{r}}=\int_{H^{r}} \boldsymbol{a} \times \overline{\boldsymbol{t}}^{l r} d H^{r},
\end{aligned}
$$

as resultantes de força e momento impostos nas laterais da casca nessa ordem, e

$$
\begin{aligned}
& \boldsymbol{n}^{\Gamma_{u}^{r}}=\int_{H^{r}} \boldsymbol{r} d H^{r} \\
& \boldsymbol{m}^{\Gamma_{u}^{r}}=\int_{H^{r}} \boldsymbol{a} \times \boldsymbol{r} d H^{r}
\end{aligned}
$$

as resultantes de reações na fronteira essencial.

Reunindo grandezas nos vetores

$$
\overline{\boldsymbol{q}}^{\Omega^{r}}=\left[\begin{array}{c}
\overline{\boldsymbol{n}}^{\Omega^{r}} \\
\overline{\boldsymbol{\mu}}^{\Omega^{r}}
\end{array}\right] \quad \overline{\boldsymbol{q}}^{\Gamma_{t}^{r}}=\left[\begin{array}{c}
\overline{\boldsymbol{n}}^{\Gamma_{t}^{r}} \\
\overline{\boldsymbol{\mu}}^{\Gamma_{t}^{r}}
\end{array}\right] \quad \boldsymbol{q}^{\Gamma_{u}^{r}}=\left[\begin{array}{c}
\boldsymbol{n}^{\Gamma_{u}^{r}} \\
\boldsymbol{\mu}^{\Gamma_{u}^{r}}
\end{array}\right],
$$

onde

$$
\begin{aligned}
& \overline{\boldsymbol{\mu}}^{\Omega^{r}}=\boldsymbol{\Gamma}^{T} \overline{\boldsymbol{m}}^{\Omega^{r}} \\
& \overline{\boldsymbol{\mu}}^{\Gamma_{t}^{r}}=\boldsymbol{\Gamma}^{T} \overline{\boldsymbol{m}}^{\Gamma_{t}^{r}} \\
& \boldsymbol{\mu}^{\Gamma_{u}^{r}}=\boldsymbol{\Gamma}^{T} \boldsymbol{m}^{\Gamma_{u}^{r}}
\end{aligned}
$$

são os pseudo-momentos energeticamente conjugados com $\dot{\boldsymbol{\theta}}$, pode-se escrever a equação (4.77) de forma mais compacta:

$$
P_{\mathrm{ext}}=\int_{\Omega^{r}}\left(\overline{\boldsymbol{q}}^{\Omega^{r}} \cdot \dot{\boldsymbol{d}}\right) d \Omega+\int_{\Gamma_{t}^{r}}\left(\overline{\boldsymbol{q}}^{\Gamma_{t}^{r}} \cdot \dot{\boldsymbol{d}}\right) d \Gamma_{t}^{r}+\int_{\Gamma_{u}^{r}}\left(\boldsymbol{q}^{\Gamma_{u}^{r}} \cdot \dot{\boldsymbol{d}}\right) d \Gamma_{u}^{r} \cdot
$$

\subsection{Forma fraca}

Similarmente a (4.75), pode-se obter o trabalho virtual das forças internas no domínio da casca por 


$$
\delta W_{\mathrm{int}}=\int_{\Omega^{r}}\left(\boldsymbol{\sigma}_{\alpha}^{r} \cdot \delta \boldsymbol{\varepsilon}_{\alpha}^{r}\right) d \Omega
$$

onde, analogamente a (4.73), $\delta \varepsilon_{\alpha}^{r}=\boldsymbol{\Psi}_{\alpha} \boldsymbol{\Delta}_{\alpha} \delta \boldsymbol{d}$ é uma variação das deformações generalizadas.

Da mesma forma, o trabalho virtual das forças externas, incluindo as reações de apoio, será

$$
\delta W_{\mathrm{ext}}=\int_{\Omega^{r}}\left(\overline{\boldsymbol{q}}^{\Omega^{r}} \cdot \delta \boldsymbol{d}\right) d \Omega+\int_{\Gamma_{t}^{r}}\left(\overline{\boldsymbol{q}}^{\Gamma_{t}^{r}} \cdot \delta \boldsymbol{d}\right) d \Gamma_{t}^{r}+\int_{\Gamma_{u}^{r}}\left(\boldsymbol{q}^{\Gamma_{u}^{r}} \cdot \delta \boldsymbol{d}\right) d \Gamma_{u}^{r}
$$

O equilíbrio da casca pode, então, ser imposto de forma fraca pelo Princípio dos Trabalho Virtuais

$$
\delta W_{\text {int }}-\delta W_{\text {ext }}=0, \quad \forall \delta \boldsymbol{d}
$$

onde $\delta \boldsymbol{d}$ é um campo de deslocamentos virtuais infinitesimais.

Tomando que as rotações impostas na fronteira da casca possam ser expressas pelos parâmetros de Euler $\overline{\boldsymbol{\theta}}$ (caso contrário, deverá ser extraído do tensor prescrito por uma inversão da relação (4.36) como descrito em Tiago[2]), podemos reuni-las com os deslocamentos no vetor

$$
\overline{\boldsymbol{d}}=\left[\begin{array}{l}
\overline{\boldsymbol{u}} \\
\overline{\boldsymbol{\theta}}
\end{array}\right]
$$

Como o Moving Least Squares não é um processo interpolatório, as condições de fronteira cinemática não podem ser atendidas a priori. Assim, serão impostas, também, de forma fraca por

$$
-\int_{\Gamma_{u}^{r}} \delta \boldsymbol{q}^{\Gamma_{u}^{r}} \cdot(\boldsymbol{d}-\overline{\boldsymbol{d}}) d \Gamma_{u}^{r}=0, \quad \forall \delta \boldsymbol{q}^{\Gamma_{u}^{r}}
$$


Esta imposição corresponde ao trabalho virtual complementar das reações na fronteira cinemática e equivale ao uso de multiplicadores de Lagrange feito por Dolbow e Belytschko em [16] para as chapas lineares, sendo que agora puderam ser identificados como as reações generalizadas na fronteira.

A combinação do Princípio dos Trabalhos Virtuais expresso em (4.86) com a imposição fraca (4.88) traduz-se no funcional híbrido de deslocamentos:

$$
\begin{aligned}
\delta W & =\int_{\Omega^{r}}\left(\boldsymbol{\sigma}_{\alpha}^{r} \cdot \delta \varepsilon_{\alpha}^{r}\right) d \Omega^{r}-\int_{\Omega^{r}}\left(\overline{\boldsymbol{q}}^{\Omega^{r}} \cdot \delta \boldsymbol{d}\right) d \Omega-\int_{\Gamma_{t}^{r}}\left(\overline{\boldsymbol{q}}_{t}^{\Gamma_{t}^{r}} \cdot \delta \boldsymbol{d}\right) d \Gamma_{t}^{r} \\
& -\int_{\Gamma_{u}^{r}}\left(\boldsymbol{q}^{\Gamma_{u}^{r}} \cdot \delta \boldsymbol{d}\right) d \Gamma_{u}^{r}-\int_{\Gamma_{u}^{r}} \delta \boldsymbol{q}^{\Gamma_{u}^{r}} \cdot(\boldsymbol{d}-\overline{\boldsymbol{d}}) d \Gamma_{u}^{r}=0 \quad \forall \delta \boldsymbol{d}, \delta \boldsymbol{q}^{\Gamma_{u}^{r}}
\end{aligned}
$$

\subsection{Recuperação das Equações de Equilíbrio}

Como na seção 3.4, podemos recuperar as equações de equilíbrio da casca a partir da forma fraca derivada. Tomando o primeiro termo de (4.89) e integrando por partes em $\boldsymbol{u}_{, \alpha}$ e em $(\boldsymbol{\Gamma} \boldsymbol{\theta})_{, \alpha}$,

$$
\begin{aligned}
& \int_{\Omega^{r}}\left(\boldsymbol{\sigma}_{\alpha}^{r} \cdot \delta \boldsymbol{\varepsilon}_{\alpha}^{r}\right) d \Omega^{r}=\int_{\Omega^{r}}\left(\boldsymbol{n}_{\alpha}^{r} \cdot \delta \boldsymbol{\eta}_{\alpha}^{r}+\boldsymbol{m}_{\alpha}^{r} \cdot \delta \boldsymbol{\kappa}_{\alpha}^{r}\right) d \Omega^{r} \\
& =\int_{\Omega^{r}}\left(\boldsymbol{n}_{\alpha}^{r} \cdot \boldsymbol{Q}^{T}\left(\delta \boldsymbol{u}_{, \alpha}+\boldsymbol{Z}_{, \alpha} \boldsymbol{\Gamma} \delta \boldsymbol{\theta}\right)+\boldsymbol{m}_{\alpha}^{r} \cdot \boldsymbol{Q}^{T}\left(\boldsymbol{\Gamma}_{, \alpha} \delta \boldsymbol{\theta}+\boldsymbol{\Gamma} \delta \boldsymbol{\theta}_{, \alpha}\right)\right) d \Omega^{r} \\
& =\int_{\Omega^{r}}\left(\boldsymbol{Q}^{T} \boldsymbol{n}_{\alpha} \cdot \boldsymbol{Q}^{T}\left(\delta \boldsymbol{u}_{, \alpha}+\boldsymbol{Z}_{, \alpha} \boldsymbol{\Gamma} \delta \boldsymbol{\theta}\right)+\boldsymbol{Q}^{T} \boldsymbol{m}_{\alpha} \cdot \boldsymbol{Q}^{T}(\boldsymbol{\Gamma} \delta \boldsymbol{\theta})_{, \alpha}\right) d \Omega^{r} \\
& =\int_{\Omega^{r}}\left(\boldsymbol{n}_{\alpha} \cdot \delta \boldsymbol{u}_{, \alpha}+\boldsymbol{n}_{\alpha} \cdot \boldsymbol{Z}_{, \alpha} \boldsymbol{\Gamma} \delta \boldsymbol{\theta}+\boldsymbol{m}_{\alpha} \cdot(\boldsymbol{\Gamma} \delta \boldsymbol{\theta})_{, \alpha}\right) d \Omega^{r} \\
& =\int_{\Omega^{r}}\left(\boldsymbol{n}_{\alpha} \cdot \delta \boldsymbol{u}_{, \alpha}\right) d \Omega^{r}+\int_{\Omega^{r}}\left(\boldsymbol{n}_{\alpha} \cdot \boldsymbol{Z}_{, \alpha} \boldsymbol{\Gamma} \delta \boldsymbol{\theta}\right) d \Omega^{r}+\int_{\Omega^{r}}\left(\boldsymbol{m}_{\alpha} \cdot(\boldsymbol{\Gamma} \delta \boldsymbol{\theta})_{, \alpha}\right) d \Omega^{r} \\
& =\int_{\Gamma^{t}} n_{\alpha}\left(\boldsymbol{n}_{\alpha} \cdot \delta \boldsymbol{u}\right) d \Gamma^{t}+\int_{\Gamma^{u}} n_{\alpha}\left(\boldsymbol{n}_{\alpha} \cdot \delta \boldsymbol{u}\right) d \Gamma^{u}-\int_{\Omega^{r}}\left(\boldsymbol{n}_{\alpha, \alpha} \cdot \delta \boldsymbol{u}\right) d \Omega^{r} \\
& +\int_{\Gamma^{t}} n_{\alpha}\left(\boldsymbol{m}_{\alpha} \cdot(\boldsymbol{\Gamma} \delta \boldsymbol{\theta})\right) d \Gamma^{t}+\int_{\Gamma^{u}} n_{\alpha}\left(\boldsymbol{m}_{\alpha} \cdot(\boldsymbol{\Gamma} \delta \boldsymbol{\theta})\right) d \Gamma^{u}-\int_{\Omega^{r}}\left(\boldsymbol{m}_{\alpha, \alpha} \cdot \boldsymbol{\Gamma} \delta \boldsymbol{\theta}\right) d \Omega^{r} \\
& +\int_{\Omega^{r}}\left(\boldsymbol{n}_{\alpha} \cdot \boldsymbol{Z}_{, \alpha} \boldsymbol{\Gamma} \delta \boldsymbol{\theta}\right) d \Omega^{r}
\end{aligned}
$$

que, voltando a (4.89), gera 


$$
\begin{aligned}
& -\int_{\Omega^{r}}\left(\boldsymbol{n}_{\alpha, \alpha}+\overline{\boldsymbol{n}}^{\Omega^{r}}\right) \cdot \delta \boldsymbol{u} d \Omega^{r}-\int_{\Omega^{r}}\left(\boldsymbol{m}_{\alpha, \alpha}+\boldsymbol{Z}_{, \alpha} \boldsymbol{n}_{\alpha}+\overline{\boldsymbol{m}}^{\Omega^{r}}\right) \cdot(\boldsymbol{\Gamma} \delta \boldsymbol{\theta}) d \Omega^{r} \\
& +\int_{\Gamma_{t}^{r}}\left(n_{\alpha} \boldsymbol{n}_{\alpha}-\overline{\boldsymbol{n}}^{\Gamma_{t}^{r}}\right) \cdot \delta \boldsymbol{u} d \Gamma_{t}^{r}+\int_{\Gamma_{t}^{r}}\left(n_{\alpha} \boldsymbol{m}_{\alpha}-\overline{\boldsymbol{m}}_{t}^{\Gamma_{t}^{r}}\right) \cdot(\boldsymbol{\Gamma} \delta \boldsymbol{\theta}) d \Gamma_{t}^{r} \\
& +\int_{\Gamma^{u}}\left(n_{\alpha} \boldsymbol{n}_{\alpha}-\boldsymbol{n}^{\Gamma_{u}^{r}}\right) \cdot \delta \boldsymbol{u} d \Gamma^{u}+\int_{\Gamma^{u}}\left(n_{\alpha} \boldsymbol{m}_{\alpha}-\boldsymbol{m}^{\Gamma_{u}^{r}}\right) \cdot(\boldsymbol{\Gamma} \delta \boldsymbol{\theta}) d \Gamma^{u} \\
& -\int_{\Gamma_{u}^{r}}^{\delta} \delta \boldsymbol{q}^{\Gamma_{u}^{r}} \cdot(\boldsymbol{d}-\overline{\boldsymbol{d}}) d \Gamma_{u}^{r}=0 \quad \forall \delta \boldsymbol{d}, \delta \boldsymbol{q}^{\Gamma_{u}^{r}}
\end{aligned}
$$

Pelo Lema Fundamental do Cálculo variacional, (4.91) só será verdadeira se

$$
\begin{gathered}
\boldsymbol{n}_{\alpha, \alpha}+\overline{\boldsymbol{n}}^{\Omega^{r}}=\boldsymbol{O}, \mathrm{em} \Omega^{r}, \\
\boldsymbol{m}_{\alpha, \alpha}+\boldsymbol{z}_{, \alpha} \times \boldsymbol{n}_{\alpha}+\overline{\boldsymbol{m}}^{\Omega^{r}}=\boldsymbol{O} \\
n_{\alpha} \boldsymbol{n}_{\alpha}-\overline{\boldsymbol{n}}^{\Gamma_{t}^{r}}=\boldsymbol{O}, \text { em } \Gamma_{t}^{r}, \\
n_{\alpha} \boldsymbol{m}_{\alpha}-\overline{\boldsymbol{m}}^{\Gamma_{t}^{r}}=\boldsymbol{O} \\
n_{\alpha} \boldsymbol{n}_{\alpha}-\boldsymbol{n}^{\Gamma_{u}^{r}}=\boldsymbol{O} \\
n_{\alpha} \boldsymbol{m}_{\alpha}-\boldsymbol{m}^{\Gamma_{u}^{r}}=\boldsymbol{O}, \text { em } \Gamma_{u}^{r} \mathrm{e} \\
\boldsymbol{u}-\overline{\boldsymbol{u}}=\boldsymbol{O} \\
\boldsymbol{\theta}-\overline{\boldsymbol{\theta}}=\boldsymbol{O}, \mathrm{em} \Gamma_{u}^{r},
\end{gathered}
$$

que são as equações do equilíbrio no domínio, nas fronteiras estática e cinemática, além da compatibilidade na fronteira cinemática. Essas são as mesmas equações obtidas por equilíbrio de uma porção infinitesimal na configuração atual da casca. 


\section{CASCAS SOB LINEARIDADE GEOMÉTRICA}

O capítulo anterior apresentou de forma sucinta a teoria de cascas apresentada em Pimenta e Campello [1]. Neste, será "linearizada", gerando uma forma fraca semelhante àquela obtida no capítulo 3 .

\subsection{Linearidade Geométrica}

A cinemática e as definições de deformações generalizadas apresentadas podem acomodar deslocamentos de qualquer magnitude (ressalvas são feitas quanto a singularidades na parametrização de Euler que podem ser contornadas com processo incrementais de composição das rotações).

O presente trabalho se propõe a particularizar a transformação efetiva para o caso de pequenos deslocamentos e pequenas rotações. É importante salientar que apenas a transformação entre a configuração inicial $\Omega^{o}$ e a atual $\Omega$ será imposta como pequena. O mapeamento inicial continua a comportar grandes deslocamentos, rotações (ainda acopladas aos deslocamentos) e deformações.

Consiste, então, em tornar o funcional apresentado em (4.89) linear nos deslocamentos $\boldsymbol{u}$ e rotações $\boldsymbol{\theta}$. Para tanto, a rotação do diretor na transformação efetiva, descrita por (4.36), é tomada com seus termos de primeira ordem em $\boldsymbol{\theta}$,

$$
\boldsymbol{Q}^{e}=\boldsymbol{I}+\operatorname{Skew}(\boldsymbol{\theta})
$$

e o deslocamento de um ponto da casca, nessa mesma transformação, será

$$
\boldsymbol{\delta}=\boldsymbol{u}+\boldsymbol{\theta} \times \boldsymbol{a}^{o} .
$$

As deformações generalizadas $\varepsilon_{\alpha}^{r}$ também precisam ser expressas como funções lineares de $\boldsymbol{u}$ e $\boldsymbol{\theta}$ : 


$$
\begin{aligned}
& \boldsymbol{\varepsilon}_{\alpha}^{r}=\left[\begin{array}{c}
\boldsymbol{\eta}_{\alpha}^{r} \\
\boldsymbol{k}_{\alpha}^{r}
\end{array}\right]=\left[\begin{array}{c}
\boldsymbol{Q}^{T} \boldsymbol{z}_{, \alpha}-\boldsymbol{e}_{\alpha}^{r} \\
\boldsymbol{k}_{\alpha}^{0 r}+\boldsymbol{k}_{\alpha}^{e r}
\end{array}\right]=\left[\begin{array}{c}
\boldsymbol{Q}^{o T} \boldsymbol{Q}^{e T}\left(\boldsymbol{z}_{, \alpha}^{o}+\boldsymbol{u}_{, \alpha}\right)-\boldsymbol{e}_{\alpha}^{r} \\
\boldsymbol{k}_{\alpha}^{o r}+\boldsymbol{Q}^{T} \boldsymbol{k}_{\alpha}^{e}
\end{array}\right] \\
& =\left[\begin{array}{c}
\boldsymbol{Q}^{o T}(\boldsymbol{I}-\boldsymbol{\Theta})\left(\boldsymbol{z}_{, \alpha}^{o}+\boldsymbol{u}_{, \alpha}\right)-\boldsymbol{e}_{\alpha}^{r} \\
\boldsymbol{k}_{\alpha}^{o r}+\boldsymbol{Q}^{o T} \boldsymbol{Q}^{e T} \boldsymbol{\Gamma} \boldsymbol{\theta}_{, \alpha}
\end{array}\right] \\
& \left.=\left[\begin{array}{c}
\boldsymbol{Q}^{o T}\left(\boldsymbol{z}_{, \alpha}^{o}+\boldsymbol{u}_{, \alpha}-\boldsymbol{\Theta} \boldsymbol{z}_{, \alpha}^{o}-\boldsymbol{\Theta} \boldsymbol{u}_{, \alpha}\right)-\boldsymbol{e}_{\alpha}^{r} \\
\boldsymbol{k}_{\alpha}^{o r}+\boldsymbol{Q}^{o T}(\boldsymbol{I}-\boldsymbol{\Theta}) \boldsymbol{\Gamma} \boldsymbol{\theta}_{, \alpha}
\end{array}\right] \quad \text { (desprezando } \boldsymbol{\Theta} \boldsymbol{u}_{, \alpha}\right) \\
& =\left[\begin{array}{c}
\boldsymbol{Q}^{o T} \boldsymbol{z}_{, \alpha}^{o}+\boldsymbol{Q}^{o T}\left(\boldsymbol{u}_{, \alpha}+\boldsymbol{z}_{, \alpha}^{o} \times \boldsymbol{\theta}\right)-\boldsymbol{e}_{\alpha}^{r} \\
\boldsymbol{k}_{\alpha}^{o r}+\boldsymbol{Q}^{o T}\left(\boldsymbol{\Gamma} \boldsymbol{\theta}_{, \alpha}-\boldsymbol{\Theta} \boldsymbol{\Gamma} \boldsymbol{\theta}_{, \alpha}\right)
\end{array}\right] \quad \quad\left(\begin{array}{c}
\text { desprezando } \boldsymbol{\Theta} \boldsymbol{\Gamma} \boldsymbol{\theta}_{, \alpha} \\
\text { tomando } \boldsymbol{\Gamma} \cong \boldsymbol{I}
\end{array}\right) \\
& =\left[\begin{array}{c}
\boldsymbol{\eta}_{\alpha}^{o r}+\boldsymbol{Q}^{o T}\left(\boldsymbol{u}_{, \alpha}+\boldsymbol{z}_{, \alpha}^{o} \times \boldsymbol{\theta}\right) \\
\boldsymbol{k}_{\alpha}^{o r}+\boldsymbol{Q}^{o T} \boldsymbol{\theta}_{, \alpha}
\end{array}\right] \\
& =\boldsymbol{\varepsilon}_{\alpha}^{o r}+\left[\begin{array}{cc}
\boldsymbol{Q}^{o T} & \boldsymbol{O} \\
\boldsymbol{O} & \boldsymbol{Q}^{o T}
\end{array}\right]\left[\begin{array}{ccc}
\boldsymbol{I} & \boldsymbol{O} & \boldsymbol{Z}_{, \alpha}^{o} \\
\boldsymbol{O} & \boldsymbol{I} & \boldsymbol{O}
\end{array}\right]\left[\begin{array}{c}
\boldsymbol{u}_{, \alpha} \\
\boldsymbol{\theta}_{, \alpha} \\
\boldsymbol{\theta}
\end{array}\right]
\end{aligned}
$$

onde $\varepsilon_{\alpha}^{o r}=\left[\begin{array}{ll}\boldsymbol{\eta}_{\alpha}^{o r} & \boldsymbol{\kappa}_{\alpha}^{o r}\end{array}\right]^{T}$ reúnem as deformações iniciais e $\boldsymbol{Z}_{, \alpha}^{o}=\operatorname{Skew}\left(\boldsymbol{z}_{, \alpha}^{o}\right)$.

Resultado idêntico pode ser encontrado expandindo-se $\varepsilon_{\alpha}^{r}$ em séries de Taylor em torno do ponto $\boldsymbol{d}=\boldsymbol{O}$ e desprezando os termos de ordem maior que $1 \mathrm{em} \boldsymbol{d}$.

$$
\begin{aligned}
\varepsilon_{\alpha}^{r} & \left.\cong \varepsilon_{\alpha}^{r}\right|_{\boldsymbol{d = 0}}+\left.\frac{\partial \boldsymbol{\varepsilon}_{\alpha}^{r}}{\partial \boldsymbol{d}}\right|_{\boldsymbol{d}=\boldsymbol{0}} \boldsymbol{d} \\
& =\boldsymbol{\varepsilon}_{\alpha}^{o r}+\left.\boldsymbol{\Psi}_{\alpha}\right|_{\boldsymbol{d = 0}} \boldsymbol{\Delta}_{\alpha} \boldsymbol{d} \\
& =\boldsymbol{\varepsilon}_{\alpha}^{o r}+\boldsymbol{\Psi}_{\alpha}^{o} \boldsymbol{\Delta}_{\alpha} \boldsymbol{d}
\end{aligned}
$$

descrevendo $\boldsymbol{\Psi}_{\alpha}^{o}=\left.\boldsymbol{\Psi}_{\alpha}\right|_{d=0}$. As deformações efetivas retro-rotacionadas $\varepsilon_{\alpha}^{e r}$ serão

$$
\begin{aligned}
& \varepsilon_{\alpha}^{r}=\varepsilon_{\alpha}^{o r}+\varepsilon_{\alpha}^{e r} \\
& \varepsilon_{\alpha}^{e r}=\boldsymbol{\Psi}_{\alpha}^{o} \Delta_{\alpha} \boldsymbol{d}
\end{aligned}
$$

As deformações virtuais são obtidas de forma semelhante, já que uma variação nas deformações só pode ser feita na deformação efetiva 


$$
\delta \varepsilon_{\alpha}^{r}=\boldsymbol{\Psi}_{\alpha}^{o} \boldsymbol{\Delta}_{\alpha} \delta \boldsymbol{d}
$$

Aplicando as linearizações feitas em (5.3) à definição das deformações efetivas retrorotacionadas (4.61),

$$
\begin{aligned}
\boldsymbol{\gamma}_{\alpha}^{\mathrm{e} r}= & J^{o-1}\left(\boldsymbol{e}_{\alpha}^{r} \cdot \boldsymbol{g}_{\beta}^{o r}\right)\left(\boldsymbol{e}_{\beta}^{r}+\boldsymbol{\gamma}_{\beta}^{r}\right)-\boldsymbol{e}_{\alpha}^{r} \\
= & J^{o-1}\left(\boldsymbol{e}_{\alpha}^{r} \cdot \boldsymbol{g}_{\beta}^{o r}\right)\left(\boldsymbol{e}_{\beta}^{r}+\boldsymbol{\eta}_{\beta}^{r}+\boldsymbol{\kappa}_{\beta}^{r} \times \boldsymbol{a}^{r}\right)-\boldsymbol{e}_{\alpha}^{r} \\
= & J^{o-1}\left(\boldsymbol{e}_{\alpha}^{r} \cdot \boldsymbol{g}_{\beta}^{o r}\right) \cdot \\
& \left(\boldsymbol{e}_{\beta}^{r}+\boldsymbol{\eta}_{\beta}^{o r}+\boldsymbol{Q}^{o T}\left(\boldsymbol{u}_{, \beta}+\boldsymbol{z}_{, \beta}^{o} \times \boldsymbol{\theta}\right)+\boldsymbol{\kappa}_{\beta}^{o r}+\boldsymbol{Q}^{o T} \boldsymbol{\theta}_{, \beta} \times \boldsymbol{a}^{r}\right)-\boldsymbol{e}_{\alpha}^{r} \\
& =J^{o-1}\left(\boldsymbol{e}_{\alpha}^{r} \cdot \boldsymbol{g}_{\beta}^{o r}\right) \cdot \\
& \left(\boldsymbol{\gamma}_{\beta}^{o r}+\boldsymbol{e}_{\beta}^{r}+\boldsymbol{Q}^{o T}\left(\boldsymbol{u}_{, \beta}+\boldsymbol{z}_{, \beta}^{o} \times \boldsymbol{\theta}\right)+\boldsymbol{Q}^{o T} \boldsymbol{\theta}_{, \beta} \times \boldsymbol{a}^{r}\right)-\boldsymbol{e}_{\alpha}^{r} \\
= & J^{o-1}\left(\boldsymbol{e}_{\alpha}^{r} \cdot \boldsymbol{g}_{\beta}^{o r}\right) \boldsymbol{Q}^{o T}\left(\boldsymbol{u}_{, \beta}+\boldsymbol{z}_{, \beta}^{o} \times \boldsymbol{\theta}+\boldsymbol{\theta}_{, \beta} \times \boldsymbol{a}^{r}\right) \\
& \quad+J^{o-1}\left(\boldsymbol{e}_{\alpha}^{r} \cdot \boldsymbol{g}_{\beta}^{o r}\right) \boldsymbol{f}_{\beta}^{o r}-\boldsymbol{e}_{\alpha}^{r} \\
= & J^{o-1}\left(\boldsymbol{e}_{\alpha}^{r} \cdot \boldsymbol{g}_{\beta}^{o r}\right) \boldsymbol{Q}^{o T}\left(\boldsymbol{u}_{, \beta}+\boldsymbol{z}_{, \beta}^{o} \times \boldsymbol{\theta}+\boldsymbol{\theta}_{, \beta} \times \boldsymbol{a}^{r}\right) \\
= & J^{o-1}\left(\boldsymbol{e}_{\alpha}^{r} \cdot \boldsymbol{g}_{\beta}^{o r}\right) \boldsymbol{Q}^{o T}\left(\boldsymbol{\eta}_{\beta}^{e}+\boldsymbol{\kappa}_{\beta}^{e} \times \boldsymbol{a}^{r}\right) \\
= & J^{o-1}\left(\boldsymbol{e}_{\alpha}^{r} \cdot \boldsymbol{g}_{\beta}^{o r}\right)\left(\boldsymbol{\eta}_{\beta}^{e r}+\boldsymbol{\kappa}_{\beta}^{e r} \times \boldsymbol{a}^{r}\right)
\end{aligned}
$$

onde foram introduzidas as deformações efetivas retro-rotacionadas

$$
\begin{aligned}
& \boldsymbol{\eta}_{\alpha}^{e r}=\boldsymbol{Q}^{o T} \boldsymbol{\eta}_{\alpha}^{e}=\boldsymbol{Q}^{o T}\left(\boldsymbol{u}_{, \alpha}+\boldsymbol{z}_{, \alpha}^{o} \times \boldsymbol{\theta}\right) \\
& \boldsymbol{\kappa}_{\alpha}^{e r}=\boldsymbol{Q}^{o T} \boldsymbol{\kappa}_{\alpha}^{e}=\boldsymbol{Q}^{o T} \boldsymbol{\theta}_{, \alpha}
\end{aligned}
$$

lineares nos deslocamentos, nas rotações e em suas derivadas.

\subsection{Material Elástico Isótropo}

Por sua vez, as tensões generalizadas $\sigma_{\alpha}^{r}$ devem ser expressas como uma função linear das deformações efetivas generalizadas $\varepsilon_{\alpha}^{e r}$ 


$$
\begin{gathered}
\boldsymbol{\sigma}_{\alpha}^{r}=\boldsymbol{D}_{\alpha \beta} \boldsymbol{\varepsilon}_{\beta}^{e r} \\
\boldsymbol{D}_{\alpha \beta}=\frac{\partial \boldsymbol{\sigma}_{\alpha}^{r}}{\partial \varepsilon_{\beta}^{e r}}=\left[\begin{array}{ll}
\frac{\partial \boldsymbol{n}_{\alpha}^{r}}{\partial \boldsymbol{\eta}_{\beta}^{e r}} & \frac{\partial \boldsymbol{n}_{\alpha}^{r}}{\partial \boldsymbol{\kappa}_{\beta}^{e r}} \\
\frac{\partial \boldsymbol{m}_{\alpha}^{r}}{\partial \boldsymbol{\eta}_{\beta}^{e r}} & \frac{\partial \boldsymbol{m}_{\alpha}^{r}}{\partial \boldsymbol{\kappa}_{\beta}^{e r}}
\end{array}\right] .
\end{gathered}
$$

Nesse trabalho, adota-se o material neo-Hookeano de Simo-Ciarlet descrito em [14] e particularizado em Pimenta e Campello [1] para imposição do estado plano de tensões na configuração atual. Conforme estes, eq. $(73,74,70)$,

$$
\begin{aligned}
\boldsymbol{\tau}_{\alpha}^{e r} & =\left[\mu+\varphi\left(\bar{J}^{e}\right)\right] \boldsymbol{e}_{\alpha}^{r}-\varphi\left(\bar{J}^{e}\right) \varepsilon_{\alpha \beta}\left(\boldsymbol{e}_{3}^{r} \times \boldsymbol{\gamma}_{\beta}^{e r}\right)+\mu \boldsymbol{\gamma}_{\alpha}^{e r} \\
\varphi\left(\bar{J}^{e}\right) & =-\mu \frac{\lambda+2 \mu}{\lambda\left(\bar{J}^{e}\right)^{3}+2 \mu \bar{J}^{e}} \\
\bar{J}^{e} & =\left(1+\boldsymbol{e}_{\alpha}^{r} \cdot \boldsymbol{\gamma}_{\alpha}^{e r}+\boldsymbol{e}_{3}^{r} \cdot \boldsymbol{\gamma}_{1}^{e r} \times \boldsymbol{\gamma}_{2}^{e r}\right)
\end{aligned}
$$

onde $\lambda$ e $\mu$ são as constantes de Lamé:

$$
\begin{aligned}
& \mu=\frac{E}{2(1+\nu)} \\
& \lambda=\frac{E \nu}{(1-2 \nu)(1+\nu)}
\end{aligned}
$$

$\bar{J}^{e}$ é uma função não linear das deformações $\gamma_{\alpha}^{e r}$. Tomando apenas seus termos lineares, $\bar{J}^{e} \cong 1+\chi$, onde $\chi=\boldsymbol{e}_{\alpha}^{r} \cdot \boldsymbol{\gamma}_{\alpha}^{e r}$. Assim, (5.10)b torna-se

$$
\varphi(\chi) \cong-\mu \frac{\lambda+2 \mu}{\lambda(1+\chi)^{3}+2 \mu(1+\chi)},
$$

que ainda não é linear em relação a $\gamma_{\alpha}^{e r}$. Uma aproximação de primeira ordem, por expansão em série de Taylor e truncamento nos termos de ordem superior, é dada por 


$$
\begin{aligned}
& \varphi(\chi) \cong \varphi(0)+\frac{d}{d \chi} \varphi(0) \chi \\
& \varphi(0)=-\mu \\
& \varphi^{\prime}(\chi)=\mu\left[\frac{\left(2 \mu+3 \lambda(\chi+1)^{2}\right)(\lambda+2 \mu)}{\left(\lambda(\chi+1)^{3}+2 \mu(\chi+1)\right)^{2}}\right], \\
& \varphi^{\prime}(0)=\mu \frac{(3 \lambda+2 \mu)}{\lambda+2 \mu} \\
& \varphi(\chi) \cong \mu\left[\frac{(3 \lambda+2 \mu)}{\lambda+2 \mu} \chi-1\right] \\
& \varphi\left(\gamma_{\alpha}^{e r}\right) \cong \frac{E}{2(1+\nu)}\left[\frac{1+\nu}{1-\nu}\left(\boldsymbol{e}_{\alpha}^{r} \cdot \boldsymbol{\gamma}_{\alpha}^{e r}\right)-1\right]
\end{aligned}
$$

A substituição de (5.13) em (5.10)a faz-se ainda com a ressalva que na segunda parcela desta, $\varphi\left(\bar{J}^{e}\right)$ já multiplica $\gamma_{\alpha}^{e r}$, devendo ser substituído por sua aproximação de ordem zero $\varphi\left(\bar{J}^{e}\right) \cong-\mu$

$$
\begin{aligned}
\boldsymbol{\tau}_{\alpha}^{e r} & =\left[\frac{E}{2(1-\nu)} \boldsymbol{e}_{\beta}^{r} \cdot \boldsymbol{\gamma}_{\beta}^{e r}\right] \boldsymbol{e}_{\alpha}^{r}+\frac{E}{2(1+\nu)} \varepsilon_{\alpha \beta}\left(\boldsymbol{e}_{3}^{r} \times \boldsymbol{\gamma}_{\beta}^{e r}\right)+\frac{E}{2(1+\nu)} \gamma_{\alpha}^{e r} \\
& =\left[\frac{E}{2(1-\nu)} \boldsymbol{e}_{\alpha}^{r} \otimes \boldsymbol{e}_{\beta}^{r}\right] \boldsymbol{\gamma}_{\beta}^{e r}+\frac{E}{2(1+\nu)} \varepsilon_{\alpha \beta} \boldsymbol{E}_{3}^{r} \boldsymbol{\gamma}_{\beta}^{e r}+\frac{E}{2(1+\nu)} \gamma_{\alpha}^{e r}
\end{aligned}
$$

Os módulos de elasticidade serão, então

$$
C_{\alpha \beta}^{e r}=\frac{\partial \boldsymbol{\tau}_{\alpha}^{e r}}{\partial \boldsymbol{\gamma}_{\beta}^{e r}},
$$

de forma que

$$
\boldsymbol{\tau}_{\alpha}^{e r}=\boldsymbol{C}_{\alpha \beta}^{e r} \boldsymbol{\gamma}_{\beta}^{e r}
$$

Explicitamente, temos 


$$
\begin{aligned}
& \boldsymbol{C}_{11}^{e r}=\frac{\partial \boldsymbol{\tau}_{1}^{e r}}{\partial \boldsymbol{\gamma}_{1}^{e r}}=\frac{E}{2(1+\nu)}\left\{\frac{(1+\nu)}{(1-\nu)}\left[\boldsymbol{e}_{1}^{r} \otimes \boldsymbol{e}_{1}^{r}\right]+\boldsymbol{I}\right\} \\
& =\frac{E}{2(1+\nu)}\left[\begin{array}{ccc}
\frac{2}{(1-\nu)} & \cdot & \cdot \\
\cdot & 1 & \cdot \\
\cdot & \cdot & 1
\end{array}\right] \\
& \boldsymbol{C}_{12}^{e r}=\frac{\partial \boldsymbol{\tau}_{1}^{e r}}{\partial \boldsymbol{\gamma}_{2}^{e r}}=\frac{E}{2(1+\nu)}\left\{\frac{(1+\nu)}{(1-\nu)}\left[\boldsymbol{e}_{1}^{r} \otimes \boldsymbol{e}_{2}^{r}\right]+\boldsymbol{E}_{3}^{r}\right\} \\
& =\frac{E}{2(1+\nu)}\left[\begin{array}{cc}
\cdot & \frac{2 \nu}{(1-\nu)} \\
1 & \cdot \\
\cdot & \cdot
\end{array}\right] \\
& \boldsymbol{C}_{21}^{e r}=\frac{\partial \boldsymbol{\tau}_{2}^{e r}}{\partial \boldsymbol{\gamma}_{1}^{e r}}=\frac{E}{2(1+\nu)}\left\{\frac{(1+\nu)}{(1-\nu)}\left[\boldsymbol{e}_{2}^{r} \otimes \boldsymbol{e}_{1}^{r}\right]-\boldsymbol{E}_{3}^{r}\right\} \\
& =\frac{E}{2(1+\nu)}\left[\begin{array}{ccc}
\cdot & 1 & . \\
\frac{2 \nu}{(1-\nu)} & \cdot & . \\
\cdot & \cdot & .
\end{array}\right] \\
& \boldsymbol{C}_{22}^{e r}=\frac{\partial \boldsymbol{\tau}_{2}^{e r}}{\partial \boldsymbol{\gamma}_{2}^{e r}}=\frac{E}{2(1+\nu)}\left\{\frac{(1+\nu)}{(1-\nu)}\left[\boldsymbol{e}_{2}^{r} \otimes \boldsymbol{e}_{2}^{r}\right]+\boldsymbol{I}\right\} \\
& =\frac{E}{2(1+\nu)}\left[\begin{array}{ccc}
1 & \cdot & \cdot \\
\cdot & \frac{2}{(1-\nu)} & \cdot \\
\cdot & \cdot & 1
\end{array}\right]
\end{aligned}
$$

Fazendo uso de (4.66), as submatrizes de $\boldsymbol{D}_{\alpha \beta}$ em (5.9) serão dadas por 


$$
\begin{aligned}
\frac{\partial \boldsymbol{n}_{\alpha}^{r}}{\partial \boldsymbol{\eta}_{\beta}^{e r}} & =\frac{\partial}{\partial \boldsymbol{\eta}_{\beta}^{e r}} \int_{H}\left(\boldsymbol{\tau}_{\alpha}^{r}\right) d \xi_{3}=\int_{H}\left(\frac{\partial \boldsymbol{\tau}_{\alpha}^{r}}{\partial \boldsymbol{\eta}_{\beta}^{e r}}\right) d \xi_{3}=\int_{H}\left(\frac{\partial \boldsymbol{\tau}_{\alpha}^{r}}{\partial \boldsymbol{\gamma}_{\gamma}^{e r}} \frac{\partial \boldsymbol{\gamma}_{\gamma}^{e r}}{\partial \boldsymbol{\eta}_{\beta}^{e r}}\right) d \xi_{3} \\
& =\int_{H}\left(\frac{\partial\left(\left(\boldsymbol{g}_{\alpha}^{o r} \cdot \boldsymbol{e}_{\delta}^{r}\right) \boldsymbol{\tau}_{\delta}^{e r}\right)}{\partial \boldsymbol{\gamma}_{\gamma}^{e r}} \frac{\partial\left(J^{o-1}\left(\boldsymbol{e}_{\gamma}^{r} \cdot \boldsymbol{g}_{\omega}^{o r}\right)\left(\boldsymbol{\eta}_{\omega}^{e r}+\boldsymbol{\kappa}_{\omega}^{e r} \times \boldsymbol{a}^{r}\right)\right)}{\partial \boldsymbol{\eta}_{\beta}^{e r}}\right) d \xi_{3} \\
& =\int_{H}\left(J^{o-1}\left(\boldsymbol{g}_{\alpha}^{o r} \cdot \boldsymbol{e}_{\delta}^{r}\right) \boldsymbol{C}_{\delta \gamma}^{e r}\left(\boldsymbol{e}_{\gamma}^{r} \cdot \boldsymbol{g}_{\beta}^{o r}\right)\right) d \xi_{3} \\
& =\boldsymbol{C}_{\delta \gamma}^{e r} \int_{H}\left(J^{o-1}\left(\boldsymbol{g}_{\alpha}^{o r} \cdot \boldsymbol{e}_{\delta}^{r}\right)\left(\boldsymbol{g}_{\beta}^{o r} \cdot \boldsymbol{e}_{\gamma}^{r}\right)\right) d \xi_{3}
\end{aligned}
$$

$$
\begin{aligned}
\frac{\partial \boldsymbol{n}_{\alpha}^{r}}{\partial \boldsymbol{\kappa}_{\beta}^{e r}} & =\frac{\partial}{\partial \boldsymbol{\kappa}_{\beta}^{e r}} \int_{H}\left(\boldsymbol{\tau}_{\alpha}^{r}\right) d \xi_{3}=\int_{H}\left(\frac{\partial \boldsymbol{\tau}_{\alpha}^{r}}{\partial \boldsymbol{\kappa}_{\beta}^{e r}}\right) d \xi_{3}=\int_{H}\left(\frac{\partial \boldsymbol{\tau}_{\alpha}^{r}}{\partial \boldsymbol{\gamma}_{\gamma}^{e r}} \frac{\partial \boldsymbol{\gamma}_{\gamma}^{e r}}{\partial \boldsymbol{\kappa}_{\beta}^{e r}}\right) d \xi_{3} \\
& =\int_{H}\left(\frac{\partial\left(\left(\boldsymbol{g}_{\alpha}^{o r} \cdot \boldsymbol{e}_{\delta}^{r}\right) \boldsymbol{\tau}_{\delta}^{e r}\right)}{\partial \boldsymbol{\gamma}_{\gamma}^{e r}} \frac{\partial\left(J^{o-1}\left(\boldsymbol{e}_{\gamma}^{r} \cdot \boldsymbol{g}_{\omega}^{o r}\right)\left(\boldsymbol{\eta}_{\omega}^{e r}+\boldsymbol{\kappa}_{\omega}^{e r} \times \boldsymbol{a}^{r}\right)\right)}{\partial \boldsymbol{\kappa}_{\beta}^{e r}}\right) d \xi_{3} \\
& =\int_{H}\left(J^{o-1}\left(\boldsymbol{g}_{\alpha}^{o r} \cdot \boldsymbol{e}_{\delta}^{r}\right)\left(-\boldsymbol{C}_{\delta \gamma}^{e r}\right)\left(\boldsymbol{e}_{\gamma}^{r} \cdot \boldsymbol{g}_{\beta}^{o r}\right) \boldsymbol{A}^{r}\right) d \xi_{3} \\
& =-\boldsymbol{C}_{\delta \gamma}^{e r} \boldsymbol{E}^{r} \int_{H}\left(J^{o-1}\left(\boldsymbol{g}_{\alpha}^{o r} \cdot \boldsymbol{e}_{\delta}^{r}\right)\left(\boldsymbol{g}_{\beta}^{o r} \cdot \boldsymbol{e}_{\gamma}^{r}\right) \xi_{3}\right) d \xi_{3}
\end{aligned}
$$

$$
\begin{aligned}
\frac{\partial \boldsymbol{m}_{\alpha}^{r}}{\partial \boldsymbol{\eta}_{\beta}^{e r}} & =\frac{\partial}{\partial \boldsymbol{\eta}_{\beta}^{e r}} \int_{H}\left(\boldsymbol{a}^{r} \times \boldsymbol{\tau}_{\alpha}^{r}\right) d \xi_{3}=\int_{H}\left(\boldsymbol{a}^{r} \times \frac{\partial \boldsymbol{\tau}_{\alpha}^{r}}{\partial \boldsymbol{\eta}_{\beta}^{e r}}\right) d \xi_{3} \\
& =\int_{H}\left(\boldsymbol{A}^{r} \frac{\partial \boldsymbol{\tau}_{\alpha}^{r}}{\partial \boldsymbol{\gamma}_{\gamma}^{e r}} \frac{\partial \boldsymbol{\gamma}_{\gamma}^{e r}}{\partial \boldsymbol{\eta}_{\beta}^{e r}}\right) d \xi_{3} \\
& =\int_{H}\left(\boldsymbol{A}^{r} \frac{\partial\left(\left(\boldsymbol{g}_{\alpha}^{o r} \cdot \boldsymbol{e}_{\delta}^{r}\right) \boldsymbol{\tau}_{\delta}^{e r}\right.}{\partial \boldsymbol{\gamma}_{\gamma}^{e r}} \frac{\partial\left(J^{o-1}\left(\boldsymbol{e}_{\gamma}^{r} \cdot \boldsymbol{g}_{\omega}^{o r}\right)\left(\boldsymbol{\eta}_{\omega}^{e r}+\boldsymbol{\kappa}_{\omega}^{e r} \times \boldsymbol{a}^{r}\right)\right)}{\partial \boldsymbol{\eta}_{\beta}^{e r}}\right) d \xi_{3} \\
& =\int_{H}\left(J^{o-1} \boldsymbol{A}^{r}\left(\boldsymbol{g}_{\alpha}^{o r} \cdot \boldsymbol{e}_{\delta}^{r}\right) \boldsymbol{C}_{\delta \gamma}^{e r}\left(\boldsymbol{e}_{\gamma}^{r} \cdot \boldsymbol{g}_{\beta}^{o r}\right)\right) d \xi_{3} \\
& =\boldsymbol{E}^{r} \boldsymbol{C}_{\delta \gamma}^{e r} \int_{H}\left(J^{o-1}\left(\boldsymbol{g}_{\alpha}^{o r} \cdot \boldsymbol{e}_{\delta}^{r}\right)\left(\boldsymbol{g}_{\beta}^{o r} \cdot \boldsymbol{e}_{\gamma}^{r}\right) \xi_{3}\right) d \xi_{3}=\left(\frac{\partial \boldsymbol{n}_{\alpha}^{r}}{\partial \boldsymbol{\kappa}_{\beta}^{e r}}\right)^{T}
\end{aligned}
$$




$$
\begin{aligned}
\frac{\partial \boldsymbol{m}_{\alpha}^{r}}{\partial \boldsymbol{\kappa}_{\beta}^{e r}} & =\frac{\partial}{\partial \boldsymbol{\kappa}_{\beta}^{e r}} \int_{H}\left(\boldsymbol{a}^{r} \times \boldsymbol{\tau}_{\alpha}^{r}\right) d \xi_{3}=\int_{H}\left(\boldsymbol{a}^{r} \times \frac{\partial \boldsymbol{\tau}_{\alpha}^{r}}{\partial \boldsymbol{\kappa}_{\beta}^{e r}}\right) d \xi_{3} \\
& =\int_{H}\left(\boldsymbol{A}^{r} \frac{\partial \boldsymbol{\tau}_{\alpha}^{r}}{\partial \boldsymbol{\gamma}_{\gamma}^{e r}} \frac{\partial \boldsymbol{\gamma}_{\gamma}^{e r}}{\partial \boldsymbol{\kappa}_{\beta}^{e r}}\right) d \xi_{3} \\
& =\int_{H}\left(\boldsymbol{A}^{r} \frac{\partial\left(\left(\boldsymbol{g}_{\alpha}^{o r} \cdot \boldsymbol{e}_{\delta}^{r}\right) \boldsymbol{\tau}_{\delta}^{e r}\right.}{\partial \boldsymbol{\gamma}_{\gamma}^{e r}} \frac{\partial\left(J^{o-1}\left(\boldsymbol{e}_{\gamma}^{r} \cdot \boldsymbol{g}_{\omega}^{o r}\right)\left(\boldsymbol{\eta}_{\omega}^{e r}+\boldsymbol{\kappa}_{\omega}^{e r} \times \boldsymbol{a}^{r}\right)\right)}{\partial \boldsymbol{\kappa}_{\beta}^{e r}}\right) d \xi_{3} \\
& =-\int_{H}\left(J^{o-1} \boldsymbol{A}^{r}\left(\boldsymbol{g}_{\alpha}^{o r} \cdot \boldsymbol{e}_{\delta}^{r}\right) \boldsymbol{C}_{\delta \gamma}^{e r}\left(\boldsymbol{e}_{\gamma}^{r} \cdot \boldsymbol{g}_{\beta}^{o r}\right) \boldsymbol{A}^{r}\right) d \xi_{3} \\
& =-\boldsymbol{E}^{r} \boldsymbol{C}_{\delta \gamma}^{e r} \boldsymbol{E}^{r} \int_{H}\left(J^{o-1}\left(\boldsymbol{g}_{\alpha}^{o r} \cdot \boldsymbol{e}_{\delta}^{r}\right)\left(\boldsymbol{e}_{\gamma}^{r} \cdot \boldsymbol{g}_{\beta}^{o r}\right) \xi_{3}^{2}\right) d \xi_{3}
\end{aligned}
$$

\subsection{Integração na Espessura}

Pode-se ver que $\boldsymbol{D}$ só é obtido após a integração na espessura. Como o objetivo deste trabalho é a obtenção de um sistema linear o mais próximo possível daquele derivado no capítulo 3, tenta-se evitar uma integração numérica na espessura.

Para os mapeamentos iniciais apresentados na seção 4.1.1, $D$ pode ser obtido analiticamente depois da suposição de que a superfície de referência é a superfície média da casca $\left(\xi_{3} \in[-h / 2 ; h / 2]\right)$,

\subsubsection{Placa Plana}

Para a placa plana, os vetores que definem a inversa da transformação inicial e o seu Jacobiano serão

$$
\boldsymbol{g}_{1}^{\text {or }}=a_{2} \boldsymbol{e}_{1}^{r}, \quad \boldsymbol{g}_{2}^{\text {or }}=a_{1} e_{2}^{r}, \quad J^{o}=a_{1} a_{2},
$$

todos constantes na espessura. Com isso, as componentes de $\boldsymbol{D}$ podem ser obtidas analiticamente como

$$
\frac{\partial \boldsymbol{n}^{r}}{\partial \boldsymbol{\eta}^{e r}}=h\left[\begin{array}{cc}
a_{2} / a_{1} \boldsymbol{C}_{11}^{e r} & \boldsymbol{C}_{12}^{e r} \\
\boldsymbol{C}_{21}^{e r} & a_{1} / a_{2} \boldsymbol{C}_{22}^{e r}
\end{array}\right],
$$




$$
\begin{gathered}
\frac{\partial \boldsymbol{n}_{\alpha}^{r}}{\partial \boldsymbol{\kappa}_{\beta}^{e r}}=\frac{\partial \boldsymbol{m}_{\alpha}^{r}}{\partial \boldsymbol{\eta}_{\beta}^{e r}}=\boldsymbol{O} \mathrm{e} \\
\frac{\partial \boldsymbol{m}_{\alpha}^{r}}{\partial \boldsymbol{\kappa}_{\beta}^{e r}}=\frac{-h^{3} a_{2}}{12 a_{1}} \boldsymbol{E}^{r} \boldsymbol{C}_{\alpha \beta}^{e r} \boldsymbol{E}^{r} .
\end{gathered}
$$

\subsubsection{Casca Cilíndrica}

Para a casca cilíndrica descrita na seção 4.1.1, a transformação inicial fica caracterizada com os vetores e o determinante

$$
\boldsymbol{g}_{1}^{o r}=\left(1-\frac{\xi_{3}}{r}\right) \boldsymbol{e}_{1}^{r}, \quad \boldsymbol{g}_{2}^{o r}=\boldsymbol{e}_{2}^{r}, \quad J^{o}=\left(1-\frac{\xi_{3}}{r}\right)
$$

com os quais, podemos determinar as componentes de $\boldsymbol{D}$. As componentes que relacionam tensões e deformações de membrana serão

$$
\begin{aligned}
\frac{\partial \boldsymbol{n}_{1}^{r}}{\partial \boldsymbol{\eta}_{1}^{e r}} & =\int_{H}\left(1-\xi_{3} / r\right) \boldsymbol{C}_{11}^{e r} d \xi_{3}=h \boldsymbol{C}_{11}^{e r} \\
\frac{\partial \boldsymbol{n}_{1}^{r}}{\partial \boldsymbol{\eta}_{2}^{e r}} & =\int_{H} \boldsymbol{C}_{12}^{e r} d \xi_{3}=h \boldsymbol{C}_{12}^{e r} \\
\frac{\partial \boldsymbol{n}_{2}^{r}}{\partial \boldsymbol{\eta}_{1}^{e r}} & =\int_{H} \boldsymbol{C}_{21}^{e r} d \xi_{3}=h \boldsymbol{C}_{21}^{e r}=\left(\frac{\partial \boldsymbol{n}_{1}^{r}}{\partial \boldsymbol{\eta}_{2}^{e r}}\right)^{T} \\
\frac{\partial \boldsymbol{n}_{2}^{r}}{\partial \boldsymbol{\eta}_{2}^{e r}} & =\int_{H} \frac{1}{\left(1-\frac{\xi_{3}}{r}\right)} \boldsymbol{C}_{22}^{e r} d \xi_{3}=r \ln \left(\frac{-h-2 r}{h-2 r}\right) \boldsymbol{C}_{22}^{e r} \stackrel{r>>h}{\longrightarrow} h \boldsymbol{C}_{22}^{e r}
\end{aligned}
$$

onde, para simplificar a obtenção de $\boldsymbol{C}_{22}^{e r}$, pode-se assumir que o raio de curvatura da casca é bem maior que sua espessura.

As componentes cruzadas, que relacionam as tensões de membrana com as curvaturas e as resultantes de momento com as deformações de membrana, são 


$$
\begin{aligned}
\frac{\partial \boldsymbol{n}_{\alpha}^{r}}{\partial \boldsymbol{\kappa}_{\beta}^{e r}} & =-\left(\boldsymbol{g}_{\alpha}^{o r} \cdot \boldsymbol{e}_{\delta}^{r}\right)\left(\boldsymbol{e}_{\gamma}^{r} \cdot \boldsymbol{g}_{\beta}^{o r}\right) \boldsymbol{C}_{\delta \gamma}^{e r} \boldsymbol{E}^{r} \int_{H} \frac{\xi_{3}}{\left(1-\frac{\xi_{3}}{r}\right)} d \xi_{3} \\
& =-\left(\boldsymbol{g}_{\alpha}^{o r} \cdot \boldsymbol{e}_{\delta}^{r}\right)\left(\boldsymbol{e}_{\gamma}^{r} \cdot \boldsymbol{g}_{\beta}^{o r}\right) \boldsymbol{C}_{\delta \gamma}^{e r} \boldsymbol{E}^{r}\left(r^{2} \ln \left(-\frac{h+2 r}{h-2 r}\right)-h r\right) \\
& \stackrel{r>>h}{\longrightarrow} \boldsymbol{O} \\
\frac{\partial \boldsymbol{m}_{\alpha}^{r}}{\partial \boldsymbol{\eta}_{\beta}^{e r}} & =\left(\frac{\partial \boldsymbol{n}_{\alpha}^{r}}{\partial \boldsymbol{\kappa}_{\beta}^{e r}}\right)^{T}
\end{aligned}
$$

mais uma vez a expressão pode ser simplificada com a consideração feita sobre a relação curvatura - raio da casca, que também se aplica à relação entre momentos e curvaturas:

$$
\begin{aligned}
\frac{\partial \boldsymbol{m}_{1}^{r}}{\partial \boldsymbol{\kappa}_{1}^{e r}} & =-\boldsymbol{E}^{r} \boldsymbol{C}_{11}^{e r} \boldsymbol{E}^{r} \int_{H} \xi_{3}^{2}\left(1-\xi_{3} / r\right) d \xi_{3}=\frac{-h^{3}}{12} \boldsymbol{E}^{r} \boldsymbol{C}_{11}^{e r} \boldsymbol{E}^{r} \\
\frac{\partial \boldsymbol{m}_{1}^{r}}{\partial \boldsymbol{\kappa}_{2}^{e r}} & =-\boldsymbol{E}^{r} \boldsymbol{C}_{12}^{e r} \boldsymbol{E}^{r} \int_{H} \xi_{3}^{2} d \xi_{3}=\frac{-h^{3}}{12} \boldsymbol{E}^{r} \boldsymbol{C}_{12}^{e r} \boldsymbol{E}^{r}=\left(\frac{\partial \boldsymbol{m}_{2}^{r}}{\partial \boldsymbol{\kappa}_{1}^{e r}}\right)^{T} \\
\frac{\partial \boldsymbol{m}_{2}^{r}}{\partial \boldsymbol{\kappa}_{2}^{e r}} & =-\boldsymbol{E}^{r} \boldsymbol{C}_{22}^{e r} \boldsymbol{E}^{r} \int_{H} \frac{\xi_{3}^{2}}{\left(1-\frac{\xi_{3} / r}{r}\right)} d \xi_{3} \\
& =-\boldsymbol{E}^{r} \boldsymbol{C}_{22}^{e r} \boldsymbol{E}^{r}\left(r^{3} \ln \left(\frac{-h / 2-r}{h / 2-r}\right)-h r^{2}\right) \cong \frac{-h^{3}}{12} \boldsymbol{E}^{r} \boldsymbol{C}_{22}^{e r} \boldsymbol{E}^{r}
\end{aligned}
$$

\subsubsection{Cascas Esféricas}

Nas cascas esféricas, os vetores $\boldsymbol{g}_{\alpha}^{o r}$ e o Jacobiano $J^{o}$ são

$$
\boldsymbol{g}_{1}^{o r}=\left(1-\frac{\xi_{3}}{r}\right) \boldsymbol{e}_{1}^{r}, \quad \boldsymbol{g}_{2}^{o r}=\sin \left(\xi_{2} / r\right)\left(1-\xi_{3} / r\right) \boldsymbol{e}_{2}^{r}, J^{o}=\sin \left(\xi_{2} / r\right)\left(1-\xi_{3} / r\right)^{2}
$$

De forma que as componentes de $\boldsymbol{D}$ serão 


$$
\begin{aligned}
& \frac{\partial \boldsymbol{n}_{1}^{r}}{\partial \boldsymbol{\eta}_{1}^{e r}}=\frac{1}{\sin \left(\xi_{2} / r\right)} h \boldsymbol{C}_{11}^{e r} \\
& \frac{\partial \boldsymbol{n}_{1}^{r}}{\partial \boldsymbol{\eta}_{2}^{e r}}=h \boldsymbol{C}_{12}^{e r}=\left(\frac{\partial \boldsymbol{n}_{2}^{r}}{\partial \boldsymbol{\eta}_{1}^{e r}}\right)^{T} \text {, } \\
& \frac{\partial \boldsymbol{n}_{2}^{r}}{\partial \boldsymbol{\eta}_{2}^{e r}}=\sin \left(\xi_{2} / r\right) h \boldsymbol{C}_{22}^{e r} \\
& \frac{\partial \boldsymbol{n}_{1}^{r}}{\partial \boldsymbol{\kappa}_{1}^{e r}}=-\boldsymbol{C}_{11}^{e r} \boldsymbol{E}^{r} \int_{H} \frac{\xi_{3}}{\sin \left(\xi_{2} / r\right)} d \xi_{3}=\boldsymbol{O} \\
& \frac{\partial \boldsymbol{n}_{1}^{r}}{\partial \boldsymbol{\kappa}_{2}^{e r}}=-\boldsymbol{C}_{12}^{e r} \boldsymbol{E}^{r} \int_{H} \xi_{3} d \xi_{3}=\boldsymbol{O} \\
& \frac{\partial \boldsymbol{n}_{2}^{r}}{\partial \boldsymbol{\kappa}_{2}^{e r}}=-\boldsymbol{C}_{22}^{e r} \boldsymbol{E}^{r} \int_{H} \xi_{3} \sin \left(\xi_{2} / r\right) d \xi_{3}=\boldsymbol{O} \\
& \frac{\partial \boldsymbol{m}_{1}^{r}}{\partial \boldsymbol{\kappa}_{1}^{e r}}=-\boldsymbol{E}^{r} \boldsymbol{C}_{11}^{e r} \boldsymbol{E}^{r} \int_{H} \frac{\xi_{3}^{2}}{\sin \left(\xi_{2} / r\right)} d \xi_{3}=-\frac{h^{3}}{12 \sin \left(\xi_{2} / r\right)} \boldsymbol{E}^{r} \boldsymbol{C}_{11}^{e r} \boldsymbol{E}^{r} \\
& \frac{\partial \boldsymbol{m}_{1}^{r}}{\partial \boldsymbol{\kappa}_{2}^{e r}}=-\boldsymbol{E}^{r} \boldsymbol{C}_{12}^{e r} \boldsymbol{E}^{r} \int_{H} \xi_{3}^{2} d \xi_{3}=-\frac{h^{3}}{12} \boldsymbol{E}^{r} \boldsymbol{C}_{12}^{e r} \boldsymbol{E}^{r}=\left(\frac{\partial \boldsymbol{m}_{2}^{r}}{\partial \boldsymbol{\kappa}_{1}^{e r}}\right)^{T} \\
& \frac{\partial \boldsymbol{m}_{2}^{r}}{\partial \boldsymbol{\kappa}_{2}^{e r}}=-\boldsymbol{E}^{r} \boldsymbol{C}_{22}^{e r} \boldsymbol{E}^{r} \sin \left(\xi_{2} / r\right) \int_{H} \xi_{3}^{2} d \xi_{3}=\frac{-h^{3} \sin \left(\xi_{2} / r\right)}{12} \boldsymbol{E}^{r} \boldsymbol{C}_{22}^{e r} \boldsymbol{E}^{r}
\end{aligned}
$$

\subsubsection{Cascas Abatidas}

O mesmo resultado (depois da consideração sobre o raio da casca cilíndrica) pode ser obtido se considerarmos os valores de $\boldsymbol{g}_{\alpha}^{o r}$ e $J^{o}$ constantes na espessura da casca. Essa é a hipótese de casca abatida (shallow shell theory), que permite que as deformações iniciais necessárias às integrações de (5.21)-(5.24) possam ser aproximadas por sua avaliação na superfície média da casca. Corresponde também a usar uma integração numérica na espessura, com apenas um ponto na quadratura.

As componentes de $\boldsymbol{D}_{\alpha \beta}$ computadas para $r>>$ são 


$$
\begin{aligned}
\frac{\partial \boldsymbol{n}_{\alpha}^{r}}{\partial \boldsymbol{\eta}_{\beta}^{e r}} & =h J^{o-1}\left(\boldsymbol{g}_{\alpha}^{o r} \cdot \boldsymbol{e}_{\delta}^{r}\right)\left(\boldsymbol{g}_{\beta}^{o r} \cdot \boldsymbol{e}_{\gamma}^{r}\right) \boldsymbol{C}_{\delta \gamma}^{e r} \\
\frac{\partial \boldsymbol{n}_{\alpha}^{r}}{\partial \boldsymbol{\kappa}_{\beta}^{e r}} & =-\int_{H} \xi_{3} d \xi_{3}\left(J^{o-1}\left(\boldsymbol{g}_{\alpha}^{o r} \cdot \boldsymbol{e}_{\delta}^{r}\right)\left(\boldsymbol{g}_{\beta}^{o r} \cdot \boldsymbol{e}_{\gamma}^{r}\right) \boldsymbol{C}_{\delta \gamma}^{e r} \boldsymbol{E}^{r}\right)=\boldsymbol{O} \\
\frac{\partial \boldsymbol{m}_{\alpha}^{r}}{\partial \boldsymbol{\eta}_{\beta}^{e r}} & =\left(\frac{\partial \boldsymbol{n}_{\alpha}^{r}}{\partial \boldsymbol{\kappa}_{\beta}^{e r}}\right)^{T}=\boldsymbol{O} \\
\frac{\partial \boldsymbol{m}_{\alpha}^{r}}{\partial \boldsymbol{\kappa}_{\beta}^{e r}} & =-\int_{H} \xi_{3}^{2}\left(J^{o-1}\left(\boldsymbol{g}_{\alpha}^{o r} \cdot \boldsymbol{e}_{\delta}^{r}\right)\left(\boldsymbol{g}_{\beta}^{o r} \cdot \boldsymbol{e}_{\gamma}^{r}\right) \boldsymbol{E}_{3}^{r} \boldsymbol{C}_{\delta \gamma}^{e r} \boldsymbol{E}_{3}^{r}\right) d \xi_{3} \\
& =-\frac{h^{3}}{12} J^{o-1}\left(\boldsymbol{g}_{\alpha}^{o r} \cdot \boldsymbol{e}_{\delta}^{r}\right)\left(\boldsymbol{g}_{\beta}^{o r} \cdot \boldsymbol{e}_{\gamma}^{r}\right) \boldsymbol{E}_{3}^{r} \boldsymbol{C}_{\delta \gamma}^{e r} \boldsymbol{E}_{3}^{r}
\end{aligned}
$$

Essa hipótese também pode ser aplicada a mapeamentos iniciais aproximados, desde que o raio de curvatura seja grande o bastante quando comparado com a espessura da casca. Outra possibilidade é o uso de uma integração numérica simples na espessura.

\subsection{Implementação em Método de Galerkin Livre de Elementos}

Até esse momento, nada se falou sobre a forma de aproximação dos deslocamentos e rotações efetivas do problema. Tal separação é feita intencionalmente de forma a permitir o uso de qualquer método para descrevê-los. Neste trabalho, a opção recai sobre os Mínimos Quadrados Móveis para as aproximações no domínio $(\boldsymbol{u}$ e $\boldsymbol{\theta}$ ) e para polinômios lineares na fronteira $\left(\boldsymbol{q}^{\Gamma_{u}}\right)$.

O domínio é coberto com partículas distribuídas de forma estruturada (alinhadas) ou pseudoaleatórias a depender do problema e a cada uma delas estarão atrelados seis parâmetros. Sendo $\boldsymbol{u}$ o vetor que reúne os parâmetros nodais de todas as partículas, teremos, $\boldsymbol{d}(\boldsymbol{x})=\boldsymbol{\Phi}^{\Omega}(\boldsymbol{x}) \boldsymbol{u}$, onde $\Phi_{i j}$ é a função de forma atrelada à partícula $j$ associada ao grau de liberdade $i$. 


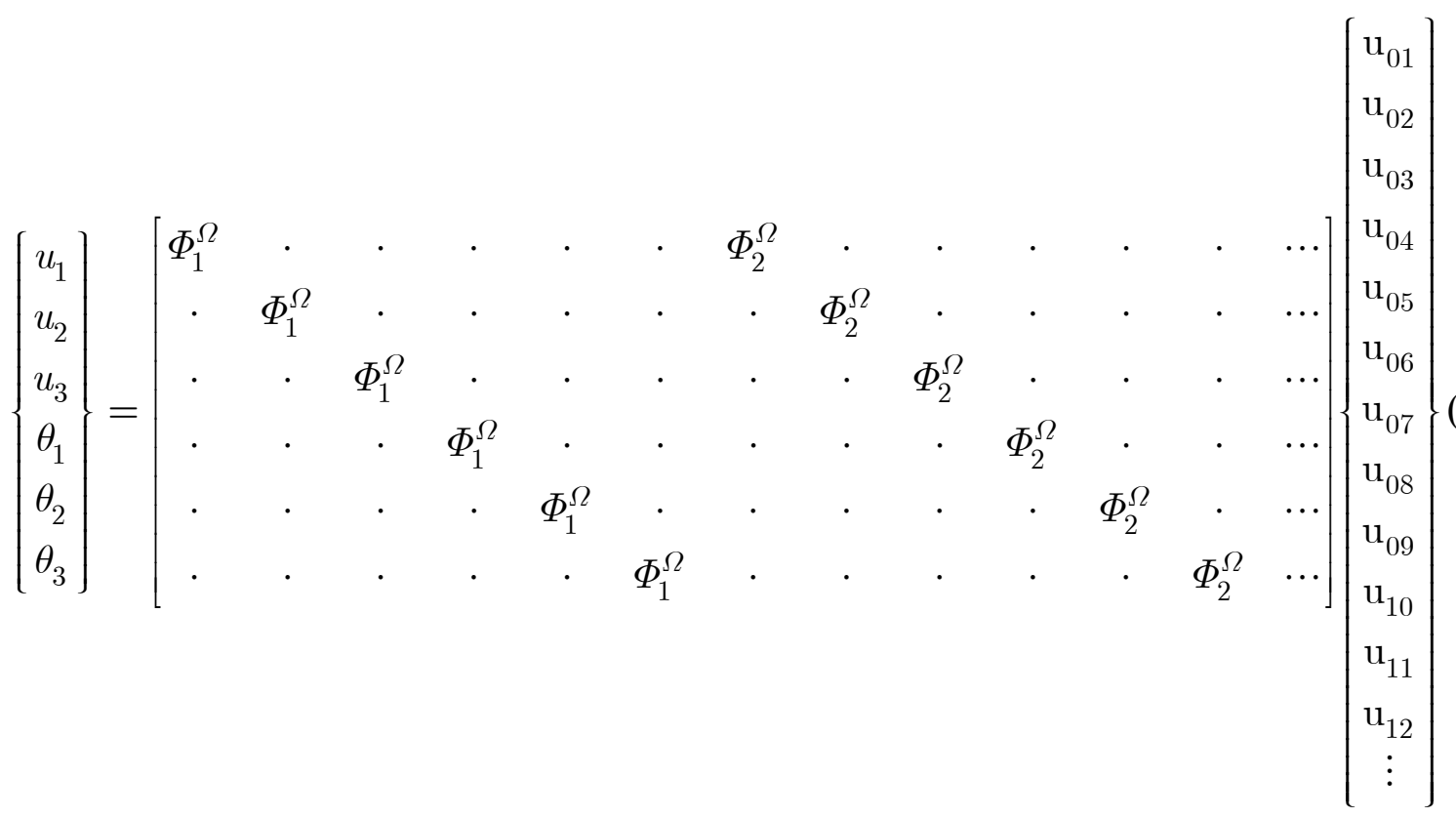

Assume-se para a aproximação das reações de apoio uma interpolação através de polinômios de Lagrange. Assim, tomando um ponto $\boldsymbol{x}_{u} \in \Gamma_{u}$, cada reação de apoio nesse ponto será aproximada por $\boldsymbol{q}^{\Gamma_{u}}=\Phi^{\Gamma_{i}}\left(\boldsymbol{x}_{u}\right) \boldsymbol{r}_{i}$ onde $\boldsymbol{r}_{i}$ são os multiplicadores de Lagrange, parâmetros nodas para a descrição das reações na fronteira. Assim, a equação (4.89) discretizada se tornará 


$$
\begin{aligned}
& \delta W=\int_{\Omega^{r}}\left(\boldsymbol{\sigma}_{\alpha}^{r} \cdot \delta \varepsilon_{\alpha}^{r}\right) d \Omega^{r}-\int_{\Omega^{r}}\left(\overline{\boldsymbol{q}}^{\Omega^{r}} \cdot \delta \boldsymbol{d}\right) d \Omega-\int_{\Gamma_{t}^{r}}\left(\overline{\boldsymbol{q}}^{\Gamma_{t}^{r}} \cdot \delta \boldsymbol{d}\right) d \Gamma_{t}^{r} \\
& -\int_{\Gamma_{u}^{r}}\left(\boldsymbol{q}^{\Gamma_{u}^{r}} \cdot \delta \boldsymbol{d}\right) d \Gamma_{u}^{r}-\int_{\Gamma_{u}^{r}} \delta \boldsymbol{q}^{\Gamma_{u}^{r}} \cdot(\boldsymbol{d}-\overline{\boldsymbol{d}}) d \Gamma_{u}^{r}=0 \quad \forall \delta \boldsymbol{d}, \delta \boldsymbol{q}^{\Gamma_{u}^{r}} \\
& \delta W=\int_{\Omega^{r}}\left(\delta \boldsymbol{\varepsilon}_{\alpha}^{r T} \boldsymbol{\sigma}_{\alpha}^{r}\right) d \Omega^{r}-\int_{\Omega^{r}}\left(\delta \mathbf{d}^{T} \overline{\mathbf{q}}^{\Omega^{r}}\right) d \Omega-\int_{\Gamma_{t}^{r}}\left(\delta \mathbf{d}^{T} \overline{\mathbf{q}}^{\Gamma_{t}^{r}}\right) d \Gamma_{t}^{r} \\
& -\int_{\Gamma_{u}^{r}}\left(\delta \mathbf{d}^{T} \mathbf{q}^{\Gamma_{u}^{r}}\right) d \Gamma_{u}^{r}-\int_{\Gamma_{u}^{r}} \delta \mathbf{q}^{\Gamma_{u}^{r}} \cdot(\mathbf{d}-\overline{\mathbf{d}}) d \Gamma_{u}^{r}=0 \\
& \delta W=\int_{\Omega^{r}}\left(\left(\boldsymbol{\Psi}_{\alpha}^{o} \boldsymbol{\Delta}_{\alpha} \boldsymbol{\delta} \mathbf{d}\right)^{T} \mathbf{D}_{\alpha \beta}\left(\boldsymbol{\Psi}_{\beta}^{o} \boldsymbol{\Delta}_{\beta} \mathbf{d}\right)\right) d \Omega^{r}-\int_{\Omega^{r}}\left(\delta \mathbf{d}^{T} \overline{\mathbf{q}}^{\Omega^{r}}\right) d \Omega \\
& -\int_{\Gamma_{t}^{r}}\left(\delta \mathbf{d}^{T} \overline{\mathbf{q}}^{\Gamma_{t}^{r}}\right) d \Gamma_{t}^{r}-\int_{\Gamma_{u}^{r}}\left(\delta \mathbf{d}^{T} \mathbf{q}^{\Gamma_{u}^{r}}\right) d \Gamma_{u}^{r} \\
& -\int_{\Gamma_{u}^{r}} \delta \mathbf{q}^{\Gamma_{u}^{r} T}(\mathbf{d}-\overline{\mathbf{d}}) d \Gamma_{u}^{r}=0 \\
& \delta W=\int_{\Omega^{r}}\left(\left(\boldsymbol{\Psi}_{\alpha}^{o} \boldsymbol{\Delta}_{\alpha} \boldsymbol{\Phi}^{\Omega} \boldsymbol{\delta} \mathbf{u}\right)^{T} \mathbf{D}_{\alpha \beta} \boldsymbol{\Psi}_{\beta}^{o} \boldsymbol{\Delta}_{\beta} \boldsymbol{\Phi}^{\Omega} \mathbf{u}\right) d \Omega^{r}-\int_{\Omega^{r}}\left(\boldsymbol{\delta} \mathbf{u}^{T} \boldsymbol{\Phi}^{\Omega T} \overline{\mathbf{q}}^{\Omega^{r}}\right) d \Omega \\
& -\int_{\Gamma_{t}^{r}}\left(\boldsymbol{\delta} \mathbf{u}^{T} \boldsymbol{\Phi}^{\Omega T} \overline{\mathbf{q}}^{\Gamma_{t}^{r}}\right) d \Gamma_{t}^{r}-\int_{\Gamma_{u}^{r}}\left(\boldsymbol{\delta} \mathbf{u}^{T} \boldsymbol{\Phi}^{\Omega T} \boldsymbol{\Phi}^{\Gamma} \mathbf{r}\right) d \Gamma_{u}^{r} \\
& -\int_{\Gamma_{u}^{r}}^{t} \delta \mathbf{r} \boldsymbol{\Phi}^{\Gamma T} \boldsymbol{\Phi}^{\Omega} \mathbf{u} d \Gamma_{u}^{r}+\int_{\Gamma_{u}^{r}} \delta \mathbf{r} \boldsymbol{\Phi}^{\Gamma} \overline{\mathbf{d}} d \Gamma_{u}^{r}=0 \\
& \delta W=\delta \mathbf{u}^{T} \int_{\Omega^{r}}\left(\left(\boldsymbol{\Psi}_{\alpha}^{o} \boldsymbol{\Delta}_{\alpha} \boldsymbol{\Phi}^{\Omega}\right)^{T} \mathbf{D}_{\alpha \beta} \boldsymbol{\Psi}_{\beta}^{o} \boldsymbol{\Delta}_{\beta} \boldsymbol{\Phi}^{\Omega}\right) d \Omega^{r} \mathbf{u}-\boldsymbol{\delta} \mathbf{u}^{T} \int_{\Omega^{r}}\left(\boldsymbol{\Phi}^{\Omega T} \overline{\mathbf{q}}^{\Omega^{r}}\right) d \Omega \\
& -\boldsymbol{\delta} \mathbf{u}^{T} \int_{\Gamma_{t}^{r}}\left(\boldsymbol{\Phi}^{\Omega T} \overline{\mathbf{q}}^{\Gamma_{t}^{r}}\right) d \Gamma_{t}^{r}-\boldsymbol{\delta} \mathbf{u}^{T} \int_{\Gamma_{u}^{r}}\left(\boldsymbol{\Phi}^{\Omega T} \boldsymbol{\Phi}^{\Gamma}\right) d \Gamma_{u}^{r} \mathbf{r} \\
& -\delta \mathbf{r}^{T} \int_{\Gamma_{u}^{r}}^{\boldsymbol{\Phi}^{\Gamma T}} \boldsymbol{\Phi}^{\Omega} d \Gamma_{u}^{r} \mathbf{u}+\delta \mathbf{r}^{T} \int_{\Gamma_{u}^{r}} \boldsymbol{\Phi}^{\Gamma T} \overline{\mathbf{d}} d \Gamma_{u}^{r}=0
\end{aligned}
$$

Como a equação é válida para $\forall \boldsymbol{\delta} \mathbf{u}, \boldsymbol{\delta} \mathbf{r}$,

$$
\begin{gathered}
\left(\int_{\Omega^{r}}\left(\left(\boldsymbol{\Psi}_{\alpha}^{o} \boldsymbol{\Delta}_{\alpha} \boldsymbol{\Phi}^{\Omega}\right)^{T} \mathbf{D}_{\alpha \beta} \boldsymbol{\Psi}_{\beta}^{0} \boldsymbol{\Delta}_{\beta} \boldsymbol{\Phi}^{\Omega}\right) d \Omega^{r}\right) \mathbf{u}+\left(-\int_{\Gamma_{u}^{r}}\left(\boldsymbol{\Phi}^{\Omega T} \boldsymbol{\Phi}^{\Gamma}\right) d \Gamma_{u}^{r}\right) \mathbf{r} \\
=\left(\int_{\Omega^{r}}\left(\boldsymbol{\Phi}^{\Omega T} \overline{\mathbf{q}}^{\Omega^{r}}\right) d \Omega+\int_{\Gamma_{t}^{r}}\left(\boldsymbol{\Phi}^{\Omega T} \overline{\mathbf{q}}_{t}^{\Gamma_{t}^{r}}\right) d \Gamma_{t}^{r}\right) \\
\left(-\int_{\Gamma_{u}^{r}} \boldsymbol{\Phi}^{\Gamma T} \mathbf{\Phi} d \Gamma_{u}^{r}\right) \mathbf{u}=\left(-\int_{\Gamma_{u}^{r}} \boldsymbol{\Phi}^{\Gamma T} \overline{\mathbf{d}} d \Gamma_{u}^{r}\right)
\end{gathered}
$$

ou 


$$
\begin{aligned}
& {\left[\begin{array}{cc}
\mathbf{K} & \mathbf{G} \\
\mathbf{G}^{T} & \mathbf{0}
\end{array}\right]\left\{\begin{array}{l}
\mathbf{u} \\
\mathbf{r}
\end{array}\right\}=\left\{\begin{array}{l}
\mathbf{f} \\
\mathbf{q}
\end{array}\right\}} \\
& \text { com } \\
& \mathbf{K}=\int_{\Omega^{r}}\left(\left(\boldsymbol{\Psi}_{\alpha}^{o} \boldsymbol{\Delta}_{\alpha} \boldsymbol{\Phi}^{\Omega}\right)^{T} \mathbf{D}_{\alpha \beta} \mathbf{\Psi}_{\beta}^{0} \boldsymbol{\Delta}_{\beta} \boldsymbol{\Phi}^{\Omega}\right) d \Omega^{r} \\
& \mathbf{G}=\int_{\Gamma_{u}^{r}}-\left(\boldsymbol{\Phi}^{\Omega T} \boldsymbol{\Phi}^{T}\right) d \Gamma_{u}^{r} \\
& \mathbf{f}=\int_{\Omega^{r}}\left(\boldsymbol{\Phi}^{\Omega T} \overline{\mathbf{q}}^{\Omega^{r}}\right) d \Omega+\int_{\Gamma_{t}^{r}}\left(\boldsymbol{\Phi}^{\Omega T} \overline{\mathbf{q}}^{\Gamma_{t}^{r}}\right) d \Gamma_{t}^{r} \\
& \mathbf{q}=\left(\int_{\Gamma_{u}^{r}}-\boldsymbol{\Phi}^{T T} \overline{\mathbf{d}} d \Gamma_{u}^{r}\right)
\end{aligned}
$$

O sistema resultante de equações é bastante similar àquele derivado no capítulo 3, com modificações na matriz constitutiva $\boldsymbol{D}$ e a inclusão das matrizes $\boldsymbol{\Psi}_{\alpha}^{o}$. Tal semelhança, um dos objetivos deste trabalho, permite a modificação do código numérico implementado na plataforma MATLAB para a resolução dos problemas de placa plana a fim de que pudesse analisar as cascas lineares. 


\subsection{Simulações Numéricas}

\subsubsection{Casca cilíndrica engastada.}

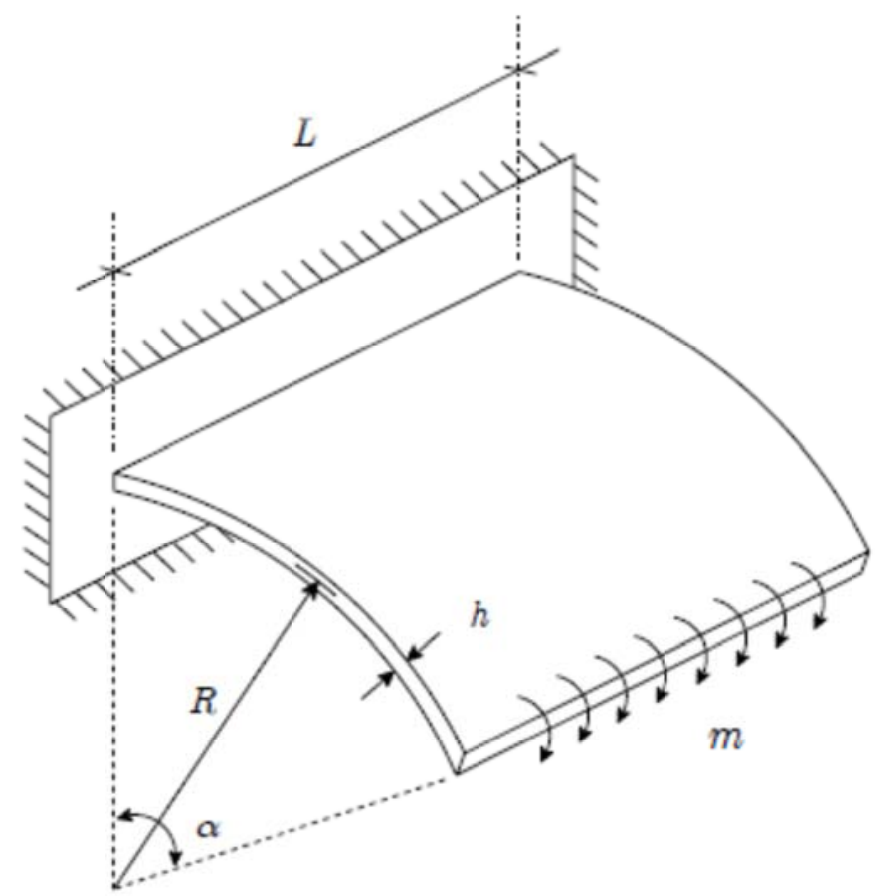

Figura 22 - Casca cilíndrica engastada (retirada de Campello [12])

A casca cilíndrica representada na Figura 22 encontra-se engastada em um bordo e com um momento aplicado no bordo oposto. Considerando um material com coeficiente de Poisson zero, tal situação apresentará um estado de flexão pura na casca.

A mesma foi modelada com diferentes números de partículas e de células de integração (cada uma usando cinco pontos de Gauss em cada direção $\xi_{\alpha}$ ), também sendo variada sua espessura. Um exemplo de distribuição de partículas e de células de integração (com os respectivos pontos de Gauss) se encontram na Figura 23. 

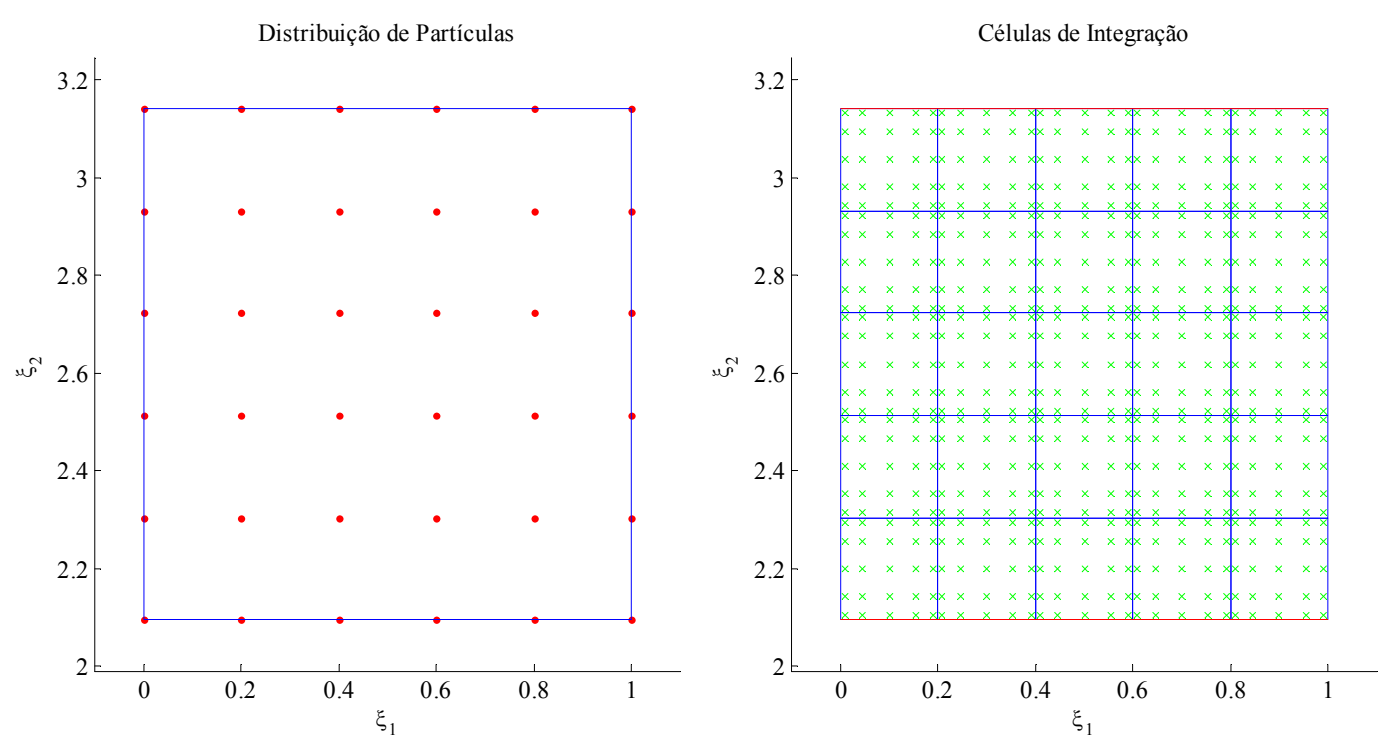

Figura 23 - Exemplo de distribuição de partículas e células de integração para casca cilíndrica com momento no bordo

Os resultados foram comparados com a solução analítica para a rotação no bordo carregado na direção axial do cilindro, dada por

$$
\theta=\frac{12 m R \alpha}{E h^{3}}
$$

sendo que foram adotados os valores de $R=2, \quad \alpha=30^{\circ}, L=1,0, \quad m=240$ e $E=2,1 \times 10^{9}$. Os resultados para diferentes distribuições de partículas são apresentados na Figura 23.

Para cascas com espessura a partir de $10^{-3}$ vezes o raio, uma base quadrática completa se mostrou suficiente para a obtenção de resultados próximos ao analítico com distribuições relativamente esparsas (próximo do mínimo necessário para que a função bi quadrática seja simplesmente definida). Contudo, para cascas mais finas, pode-se perceber a presença do travamento de membrana, bastante amenizado com a simples ampliação da base polinomial para o grau 3. 


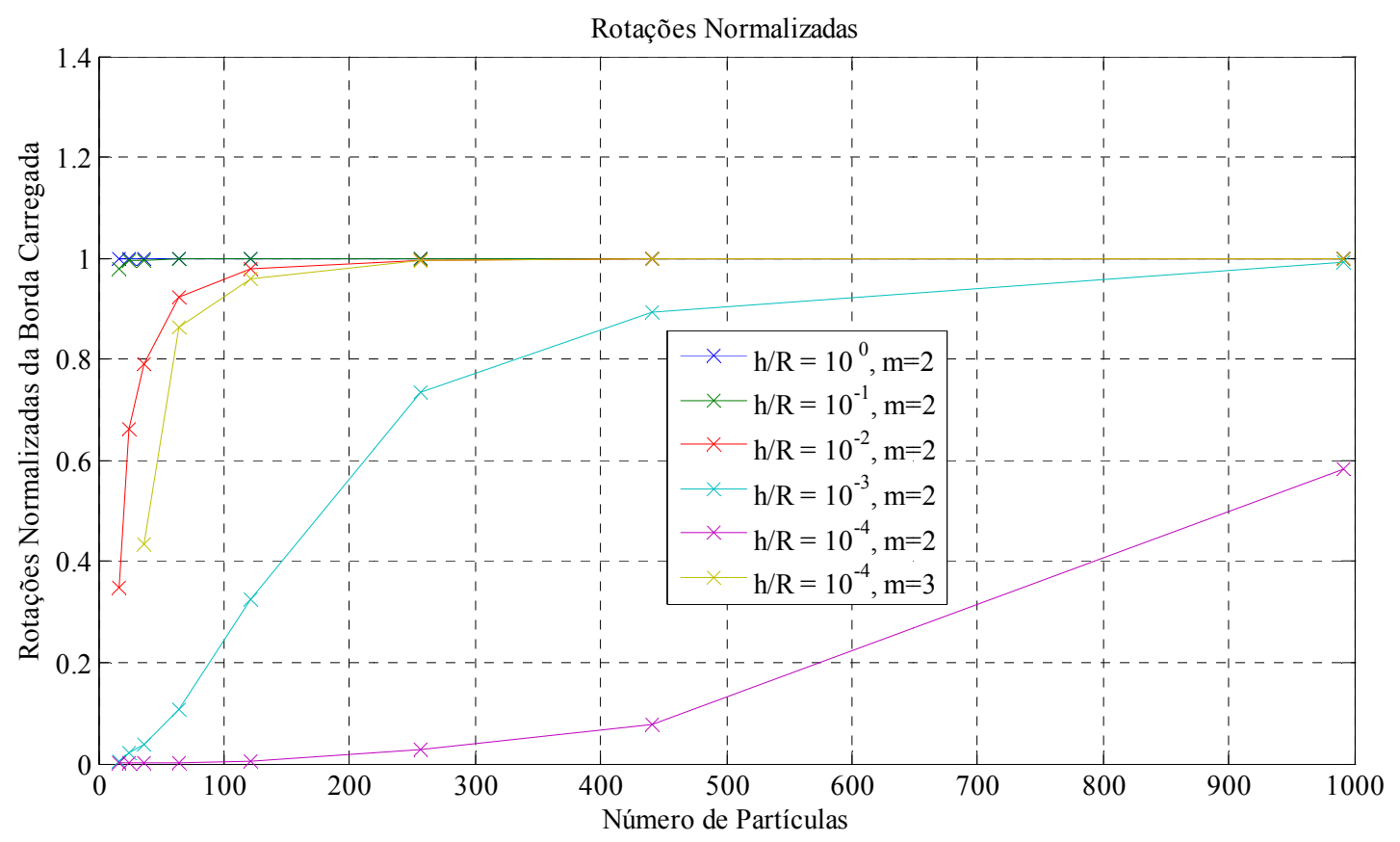

Figura 24 - Resultados da casca cilíndrica com momento no bordo

As configurações inicial e deformada (com os deslocamentos amplificados para uma melhor visualização) são representadas na Figura 25, sendo aquela a superfície monocromática azul e esta a superfície em cores, que representam o módulo dos deslocamentos.

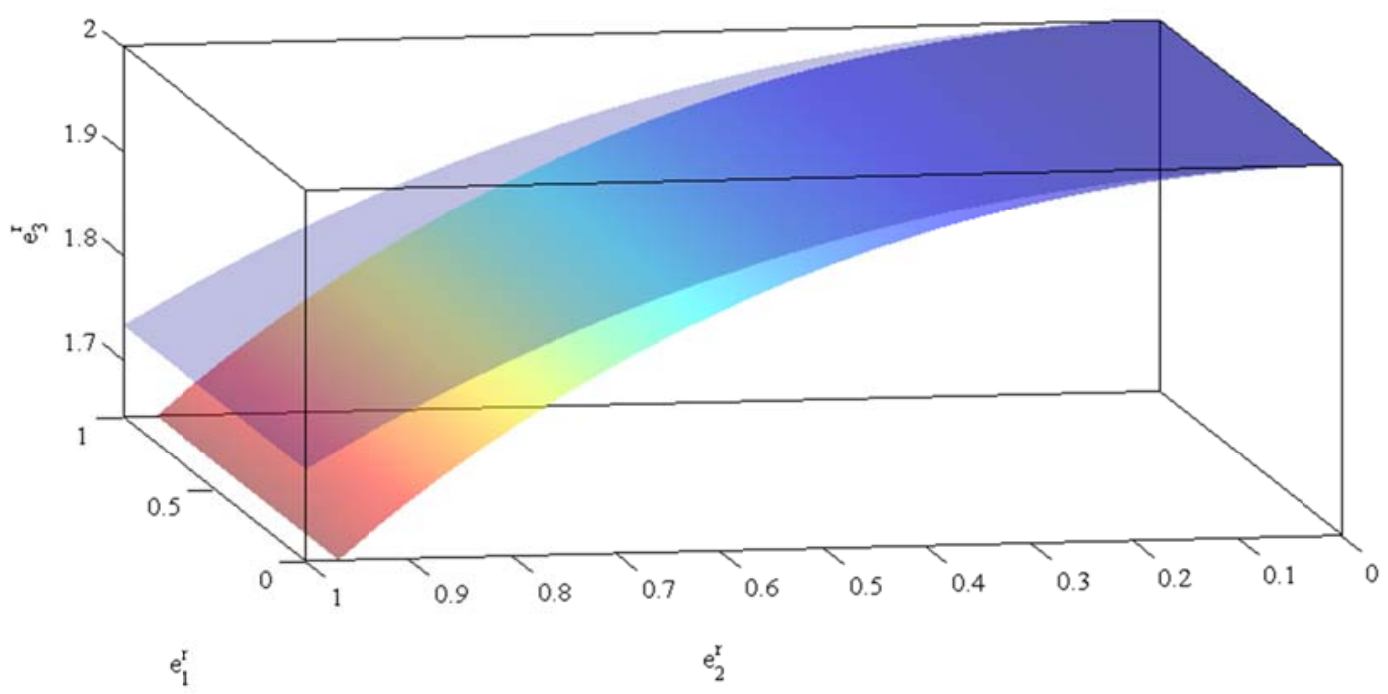

Figura 25 - Configuração inicial e deformada da casca cilindrica com momento no bordo 
As deformações generalizadas são apresentadas na Figura 26 para a distribuição de partículas e de células de integração representadas na Figura 23 e espessura da casca tal que $h / R=10^{-2}$.
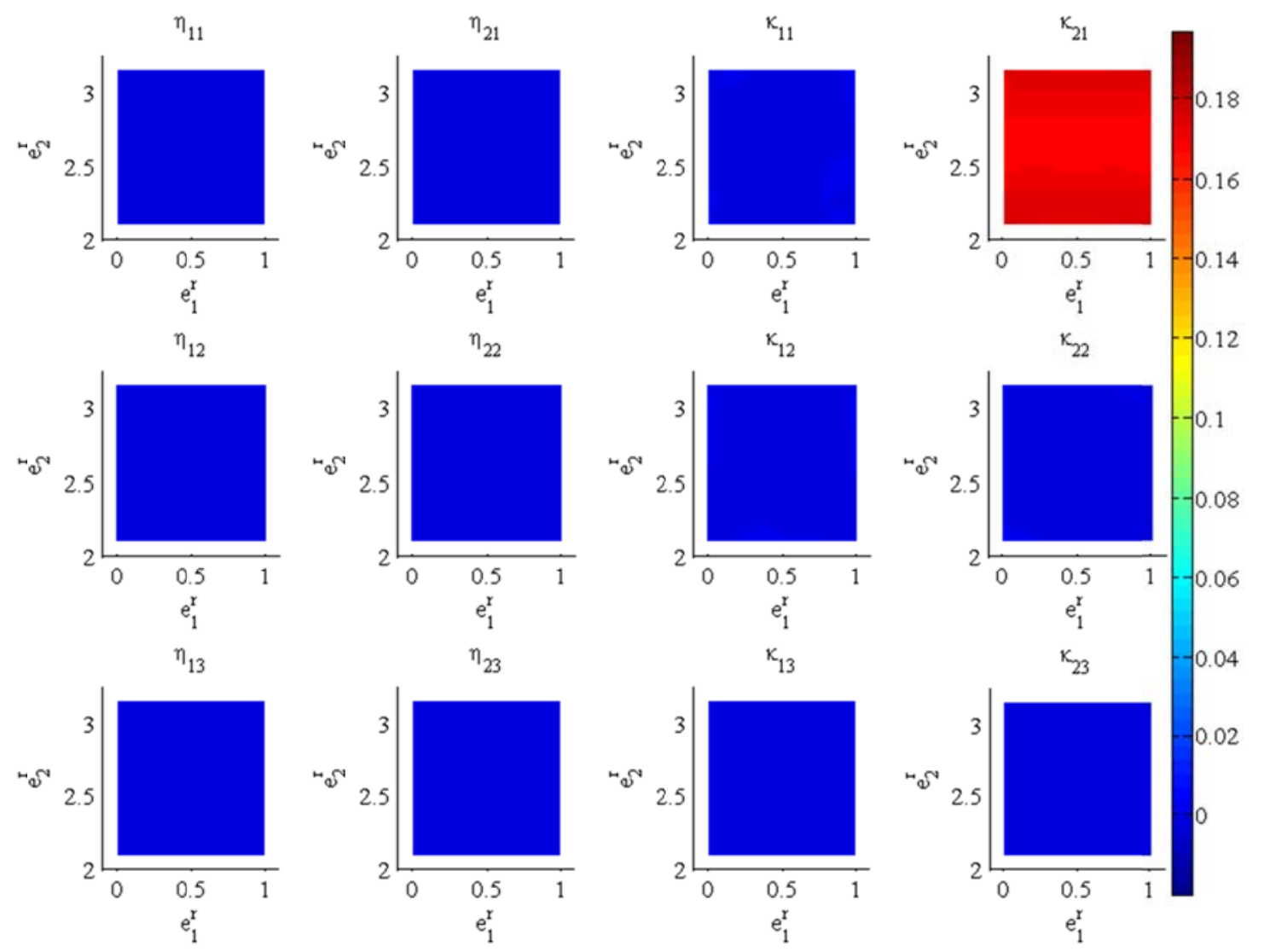

Figura 26 - Deformações generalizadas da casca cilíndrica com momento no bordo

Como esperado, essa casca só possui uma deformação não nula, a variação de $\theta_{1}$ ao longo da direção tangencial do cilindro. É patente também a suavidade do resultado.

\subsubsection{Casca cilíndrica amassada}

O exemplo a seguir foi retirado de Simo, Fox e Rifai [19] e está representado na Figura 27. Foi analisado também para o caso linear por Campello [12]. Consiste em um cilindro com diafragmas em suas extremidades que restringem os deslocamentos em $\boldsymbol{e}_{2}^{r}$ e $\boldsymbol{e}_{3}^{r}$. Por simetria, apenas $1 / 8$ do cilindro é mostrado no esquema e modelado. A carga $P$ é 1 no modelo inteiro, $\operatorname{logo} 1 / 4$ quando adotadas as condições de simetria.

As características do material e da geometria adotada são: 


$$
h=3, E=3 \times 10^{6}, \nu=0,3, R=300, L=600
$$

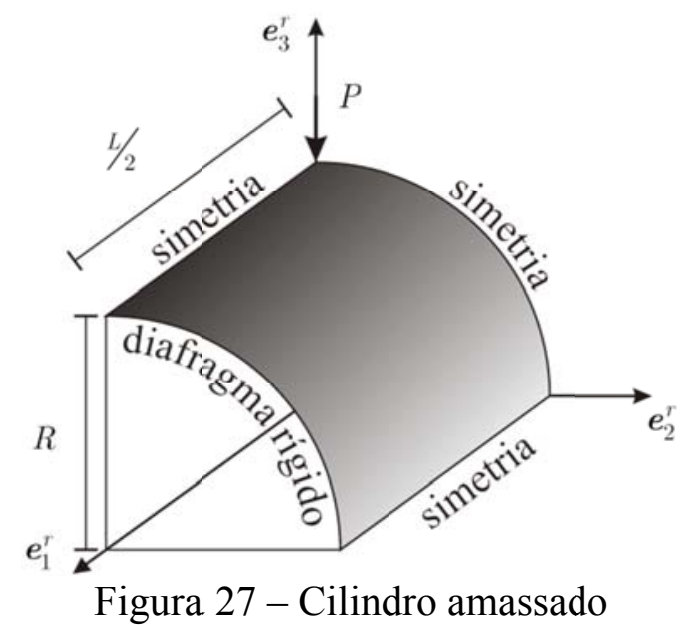

A aproximação é baseada em distribuições uniformes de partículas e a integração um número variável de células, de forma similar ao exemplo 3.7.2, sendo que o domínio $\Omega^{r}$ agora será retangular:

$$
\begin{aligned}
& 0 \leq \xi_{1} \leq \frac{L}{2} \\
& 0 \leq \xi_{2} \leq \frac{\pi}{2} r
\end{aligned}
$$

e 5 pontos de integração são utilizados em cada célula em cada direção.

A aproximação foi feita com uma base completa do quarto grau e o parâmetro de ampliação do suporte das partículas foi tomado 1,75 . O resultado para diferentes números de partículas foi normalizado em relação à resposta analítica presente em Simo, Fox e Rifai [19] $w_{\max }=-1.82488 \times 10^{-5}$ e encontra-se na Figura 28 . 


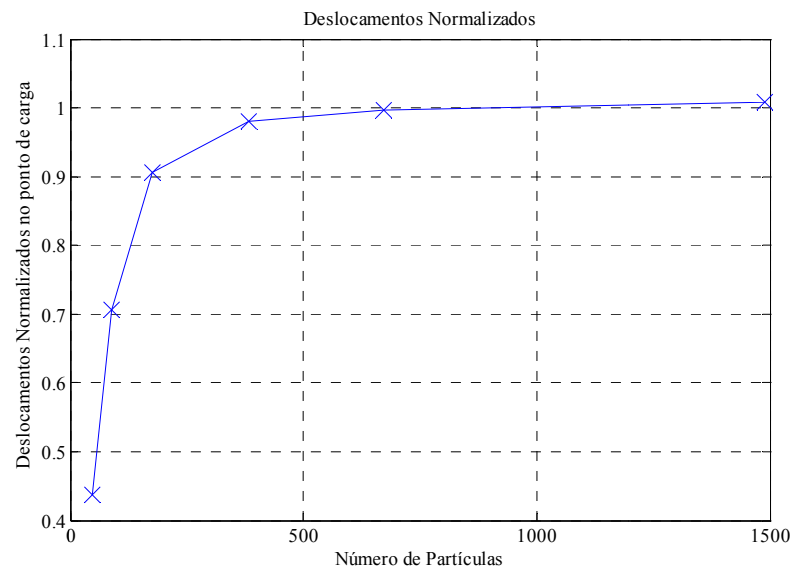

Figura 28 - Deslocamentos Normalizados para Cilindro Amassado

Os resultados são satisfatórios, haja vista que no texto de que foi retirado é considerado o mais exigente para um modelo de casca. No mesmo, a malha mais refinada aparenta ter cerca de 300 nós de Elementos Finitos e obtém resultado em torno de 0,95, algo similar ao alcançado nessa simulação com número similar de partículas. A distribuição mais fina provê um resultado diferente apenas $0,90 \%$ do tomado como referência.

As configurações inicial e deformada são representadas na Figura 29 sendo aquela a superfície monocromática azul e esta a superfície em cores, que representam o módulo dos deslocamentos. Ao lado, a reconstituição do modelo original com a consideração das simetrias.
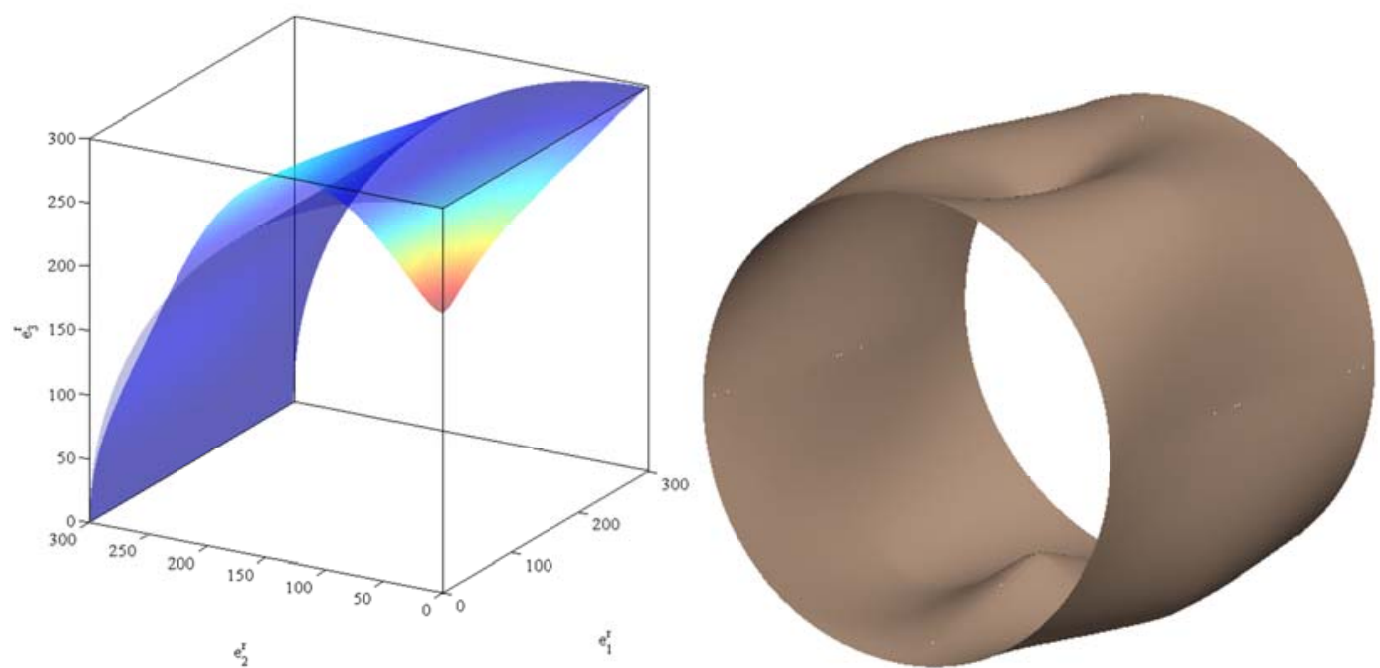

Figura 29 - Deformada do cilindro amassado (deslocamentos ampliados em $5 \times 10^{6}$ ) 


\subsubsection{Hemisfério com orifício}

A casca esférica da Figura 30 também foi retirada de Simo, Fox e Rifai [19], onde recebe o nome de Hemispherical shell with $18^{\circ}$ hole. A carga $P$ será igual a 1 no quarto de hemisfério.

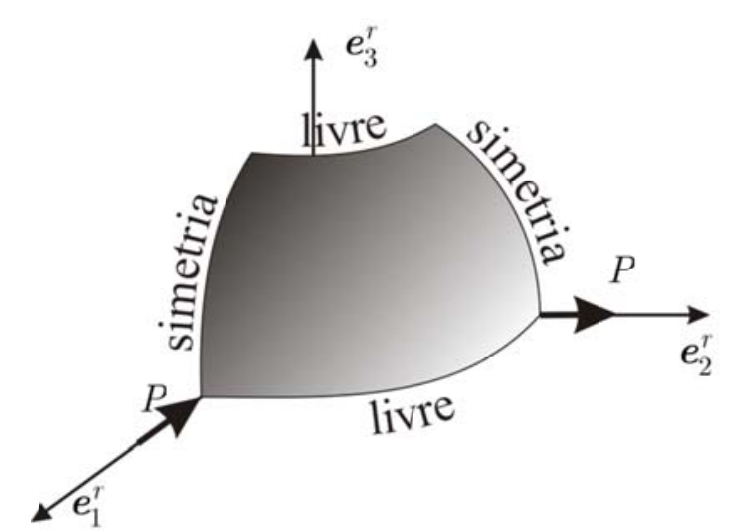

Figura 30 - Casca hemisférica com orifício

As demais propriedades geométricas são:

$$
\begin{array}{llrl}
h & =0,04 & E=6,825 \times 10^{7} & \nu=0,3 \\
R & =10 & \text { e } \quad \alpha=18^{\circ} &
\end{array}
$$

A análise foi feita com 6 diferentes distribuições de partículas e número de células de integração. Para cada análise, era gerada uma malha retangular estruturada que serviu para a integração (feita com 9 pontos por célula). Às posições dos nós, foi adicionada uma pequena perturbação de forma que as partículas não mais ficassem alinhadas. Dois exemplos de distribuição de partículas e células de integração são mostrados na Figura 31.

O fator de amplificação do domínio das partículas foi $d_{\max }=1,5$ e um polinômio bi-quíntico foi utilizado para composição da base de aproximação. 
Partículas e Integração

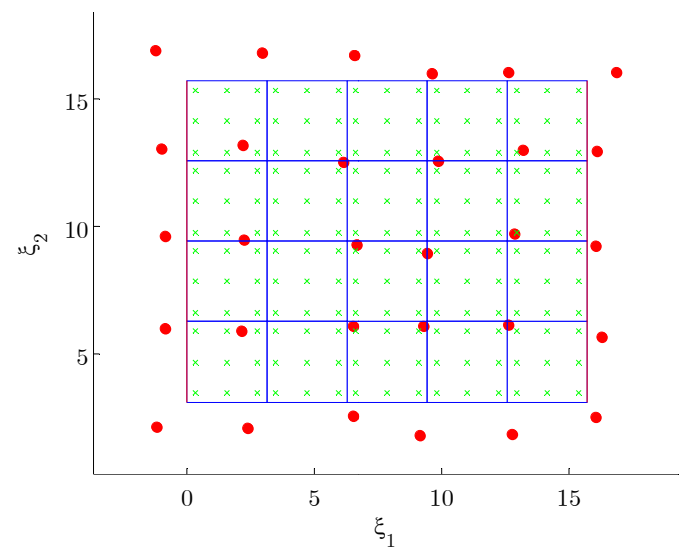

Partículas e Integração

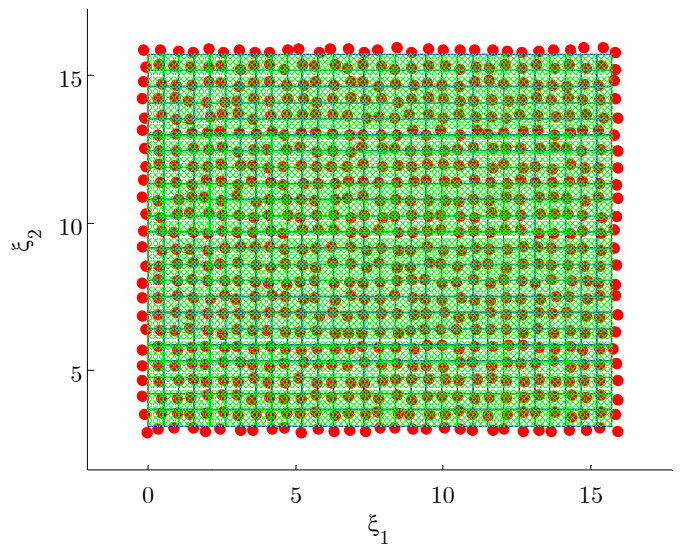

Figura 31 - Distribuições de partículas para cilindro com orifício

O deslocamento foi normalizado com relação à resposta de referência presente em [19], 0,093 e os resultados podem ser vistos na Figura 32. A Figura 33 apresenta a configuração deformada da estrutura, com os deslocamentos ampliados para uma mais fácil visualização.

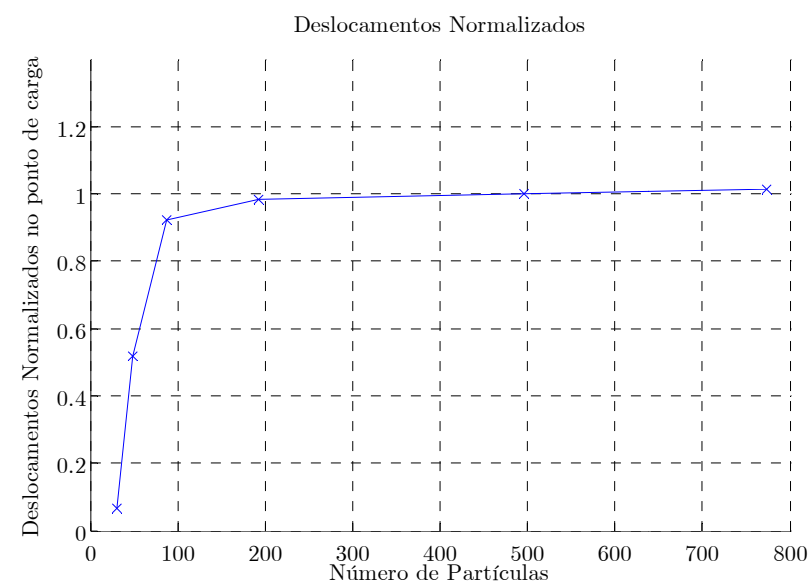

Figura 32 - Resultados para cilindro com orifício
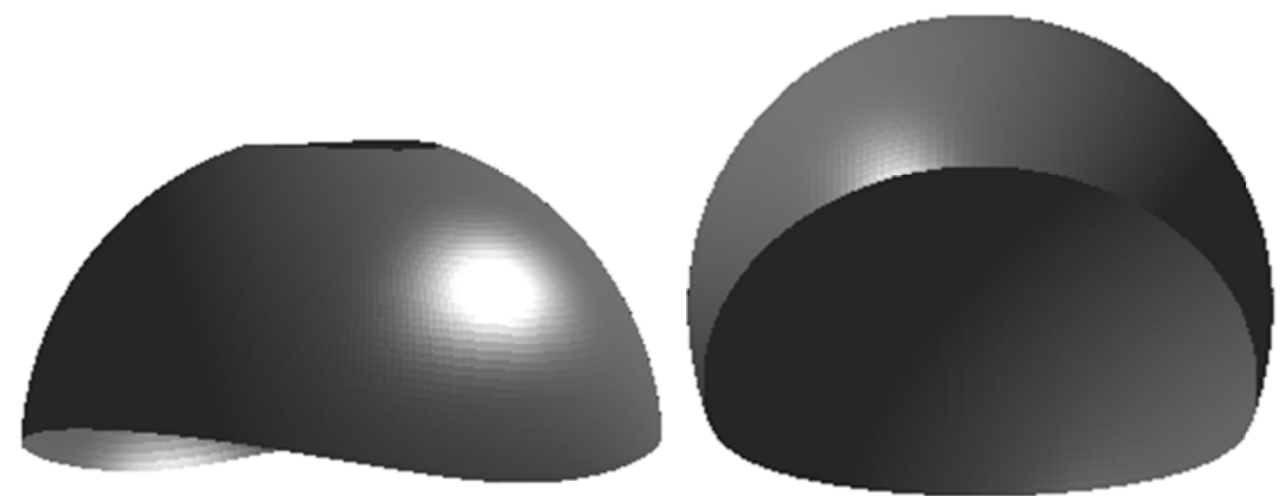

Figura 33 - Deformada do cilindro com orifício 


\subsubsection{Hemisfério Completo}

O trabalho de onde foi tirado o exemplo anterior, também propõe seu estudo sem o orifício, como na Figura 34.

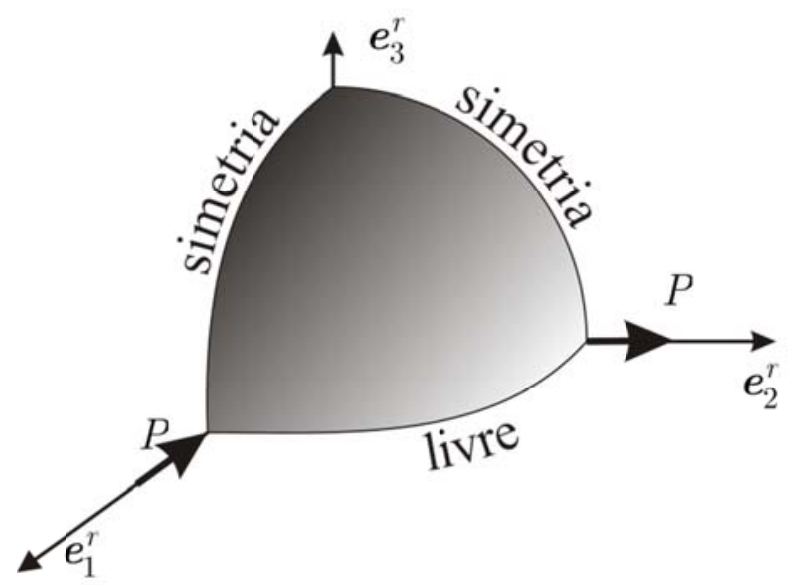

Figura 34 - Hemisfério Completo

As propriedades geométricas e de carga se repetem:

$$
\begin{array}{lll}
h=0,04 & E=6,825 \times 10^{7} & \nu=0,3 . \\
R=10 & \text { e } \quad P=1
\end{array}
$$

O mesmo método de confecção da distribuição de partículas é empregado, e continua-se usando um polinômio completo de grau cinco como base, com $d_{\max }=1,5$.
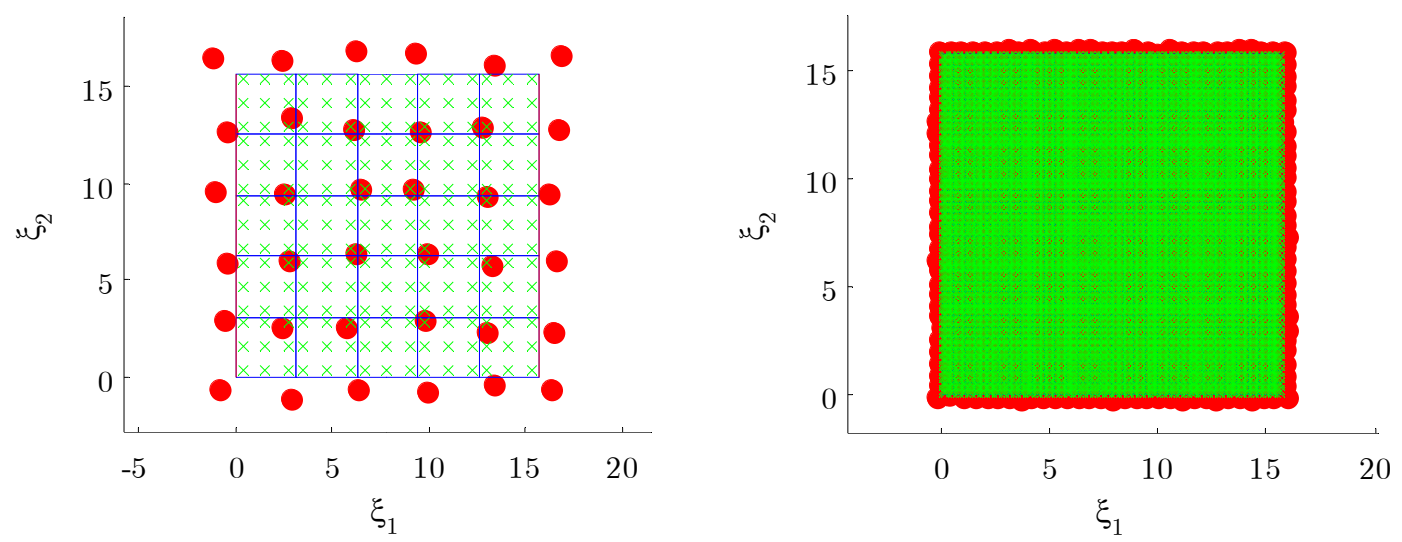

Figura 35 - Hemisfério Completo - distribuições de partículas 
Os deslocamentos dos pontos de aplicação da carga, normalizados à resposta provida em [19] de 0,0924, para as diferentes distribuições de partículas, são:

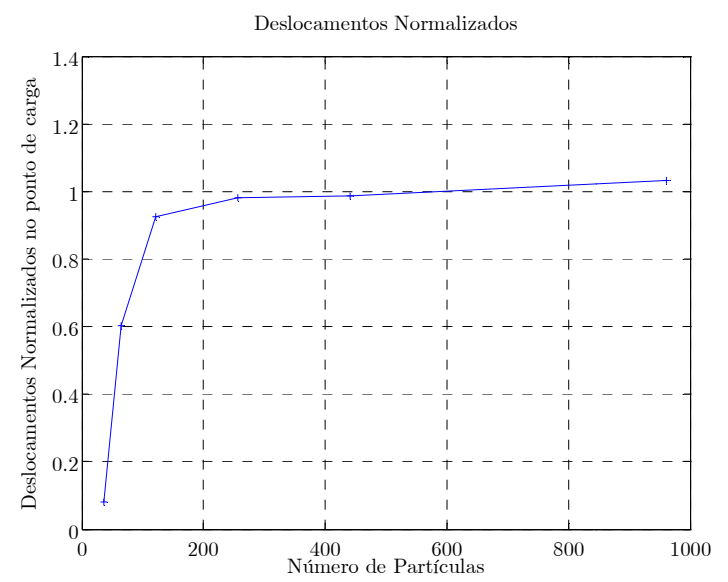

Figura 36 - Hemisfério Completo - distribuições de partículas
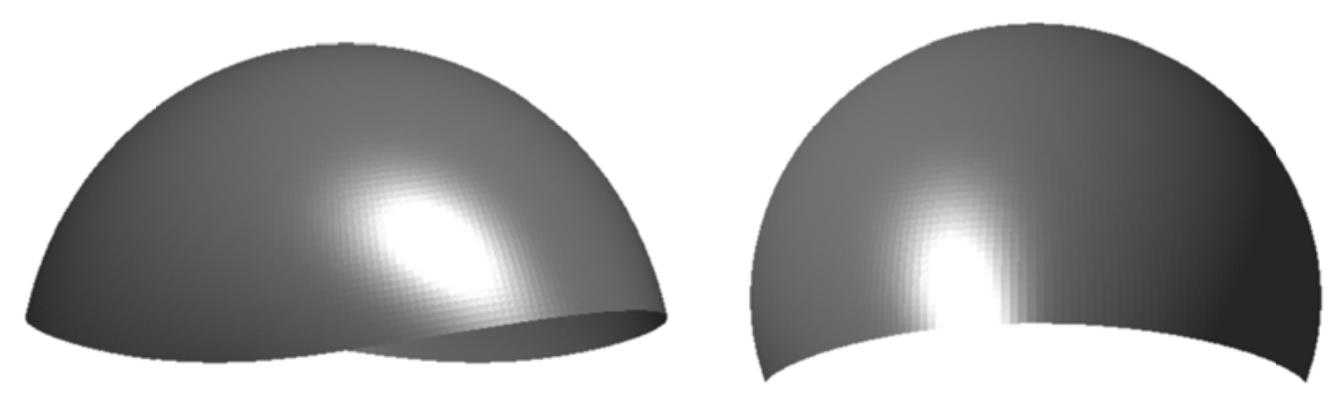

Figura 37 - Hemisfério Completo - configurações deformadas (deslocamentos amplificados em $10^{6}$ vezes)

A resposta mais uma vez é satisfatória. Um problema poderia surgir quando da análise do topo dessa casca. Aquele ponto corresponde, na configuração de referência, a uma reta $\left(\xi_{2}=0\right)$. As restrições feitas a qualquer ponto dessa reta se traduzirão a uma restrição no topo do hemisfério e não geram problemas no modelo. 


\section{CONCLUSÕES}

Os Moving Least Squares formam uma ferramenta bastante útil na mecânica computacional. A suavidade das respostas obtidas, mesmo para grandes gradientes, se prova de grande valor para o estudo das cascas, que perdem o caráter facetado imposto pelas aproximações baseadas em elementos.

Talvez seja esse o grande campo de aplicação para essa aproximação, onde tal característica justifica o maior custo computacional na avaliação das funções de forma e na resolução dos sistemas lineares, que perdem parte de sua esparsidade. A análise do contato também aparece na bibliografia como um campo onde a suavidade teria um papel importante.

A maleabilidade do método também foi explorada. Com ele, o enriquecimento das funções de forma é bastante direto, já que mais termos dos polinômios podem ser facilmente inseridos na base de aproximação. Uma análise da melhora da resposta do método quando se introduz uma leve distorção na distribuição de partículas, que deixam de estar alinhadas, poderia ter sido mais explorada, mas em quase todos os exemplos foi preterida por uma maior facilidade em gerar distribuições mais densas.

Por sua vez, a teoria de cascas apresentada, especialmente em sua versão não linear, possui um campo de aplicação bastante extenso. Seu forte apelo geométrico nas definições, a simplicidade da notação e fácil adaptação a qualquer sistema de aproximação já foi explorada em Método dos Elementos Finitos e Método de Galerkin Livre de Elementos (EFG), estando pronta a ser estendida a outros tipos de função de forma.

A derivação de uma teoria linear consistente também foi alcançada. A aproximação de primeira ordem foi imposta em deformações generalizadas consistentemente definidas, mantendo-as como tal e de forma a destacar em que pontos desprezaram-se contribuições de ordem superior. $\mathrm{O}$ mesmo rigor foi aplicado à linearização da relação constitutiva, que como esperado levou à Lei de Hooke generalizada. 
O objetivo de se chegar a uma forma fraca similar às placas sob linearidade geométrica foi atendido, o que foi revelado na simples adaptação do sistema computacional direcionado às placas para resolver as cascas. É interesse do autor verificar se o mesmo resultado é obtido com a consideração de pequenos deslocamentos e rotações efetivos já na cinemática, sem o uso da teoria geometricamente exata.

As respostas numéricas foram bastante satisfatórias. Mesmo distribuições grosseiras se aproximaram dos resultados de referência e, salvo em um caso, distribuições densas o melhoravam.

O funcional híbrido de deslocamentos derivado exigiu uma aproximação das reações generalizadas da fronteira cinemática. Por um lado, a obtenção dessas reações já na resolução do sistema linear é interessante. Por outro, o problema perde o sentido de minimização de energia, o que dificulta a estimativa do erro cometido.

A teoria também poderia ser explorada para outros mapeamentos iniciais. Uma aplicação interessante pode ser feita com um mapeamento inicial aproximado a partir de pontos coletados em uma superfície existente, ou descrita por funções utilizadas por software de Computer Aided Design (CAD). 


\section{REFERÊNCIAS BIBLIOGRÁFICAS}

[1] PIMENTA, P. M., CAMPELLO, E. M. B. Shell curvature as an initial deformation: A geometrically exact finite element approach. International Journal for Numerical Methods in Engineering. Wiley InterScience, 2009.

[2] TIAGO T F, Carlos Manuel. Meshless methods: Extending the linear formulation and its generalization to geometrically exact structural analysis. Tese de Doutorado. Instituto Superior Técnico da Universidade Técnica de Lisboa. Lisboa, 2007.

[3] LI, Shaofan, LIU, Wing Kam. Meshfree Particle Methods. Springer. Berkeley and Evanston, EUA, 2004.

[4] LIU, G. R., GU, Y. T. An Introduction to Meshfree Methods and their Programming. Springer, 2005.

[5] TIMOSHENKO, S. Theory of Elasticity. $3^{\text {rd }}$ Ed. McGraw-Hill, 1970.

[6] TIMOSHENKO, S. Strength of Materials. Part I: Elementary Theory and Problems. $2^{\text {nd }}$ Ed. D. Van Nostrand, 1940.

[7] TIMOSHENKO, S., WOINOWSKY-KRIEGER, S., Theory of Plates and Shells. $2^{\text {nd }}$ Ed. McGraw-Hill, 1987.

[8] PIMENTA, P. M. On a geometrically-exact finite-strain shell model. Proceedings of the $3^{\text {rd }}$ Pan-American Congress on Applied Mechanics, III (PACAM), São Paulo, 1993.

[9] PIMENTA, P. M. Geometrically exact analysis of initially curved rods. In: Advances in Computational Techniques for Structural Engineering. Topping H. V. (editor), vol. 1. Civil-Comp: Edinburgh, Reino Unido, 1996.

[10] WANG, C. M., REDDY, J. N., LEE, K. H. Shear Deformable Beams and Plates Relationships with Classical Solutions. Elsevier. Oxford, 2000.

[11] CAMPEllo, E. M. B., PIMENTA, P. M., WRIGGERS, P. A triangular finite shell element based on a fully nonlinear shell formulation. Computational Mechanics, 31(6), 2003. 
[12] CAMPELlo, E. M. B. Modelos não-lineares de casca em elasticidade e elastoplasticidade com grandes deformações: teoria e implementação em elementos finitos. Tese de doutorado. Escola Politécnica da Universidade de São Paulo. São Paulo, 2005.

[13] SIMO, J. C., HUGHES, T. J. R. Computational Inelasticity. Springer-Verlag. New York, 1998.

[14] CIARLET, P. J. Mathematical Elasticity, vol.1. North Holland. Amsterdam, 1988.

[15] BELYTSCHKO, T., Y. Y. Lu, L. Gu. Element-Free Galerkin Methods. International Journal for Numerical Methods in Engineering, 37. Wiley InterScience, 1994.

[16] DOLBOW, J., BELYTSCHKO, T. An Introduction to Programming the Meshless Element Free Galerkin Method. Archives of Computational Methods in Engineering, 5, 1998.

[17] DOLBOW, J., BELYTSCHKO, T. Numerical integration of the Galerkin weak form in meshfree methods. Computational Mechanics, 23. Springer-Verlag, 1999.

[18] KRYSL, P., BELYTSCHKO, T. Analysis of thin plates by the element-free Galerkin method. Computational Mechanics, 17(1-2), 1995.

[19] SIMO, J. C., FOX, D. D., RIFAI, M. S. On a stress resultant geometrically exact shell model. Part II: the linear theory; computational aspects. Computer Methods in Applied Mechanics and Engineering, 73(1), 1989.

[20] NAYROLES, B., TOUZOT, G., VILLON, P. Generalizing the finite element method: Diffuse approximation and diffuse elements. Computational Mechanics, 10. SpringerVerlag, 1992.

[21] ARGYRIS, J. H., DUNNE, P. C., SCHARPF, D. W. On large displacement-small strain analysis of structures with rotational degrees of freedom. Computer Methods in Applied Mechanics and Engineering, 14(3), 1978.

[22] BelytschKo, T., KRONGauZ, Y., ORGAN, D., FlEMing, M., Krysl, P. Meshless methods: An overview and recent developments. Computer Methods in Applied Mechanics and Engineering, 139(1-4), 1996. 
[23] BREITKOPF, P., HUERTA, A. (Editors). Meshfree and Particle Based Approaches in Computational Mechanics. Kogan Page Science, London, 2004.

[24] BREITKOPF, P., RASSINEUX, A., TOUZOT, G., VILLON, P. Explicit form and efficient computation of MLS shape functions and their derivatives. International Journal for Numerical Methods in Engineering, 48(3), 2000.

[25] CAMPELlO, E. M. B., PIMENTA, P. M., WRIGGERS, P. A geometrically-exact finite strain shell model for the analysis of initially curved shells. Sixth World Congress on Computational Mechanics Second Asian-Pacific Congress on Computational Mechanics. Tsinghua University Press \& Springer-Verlag, Beijing, China, 2004.

[26] DONNING, B. M., LIU, W. K. Meshless methods for shear-deformable beams and plates. Computer Methods in Applied Mechanics and Engineering, 152(1-2), 1998.

[27] EASON, E. D. A review of least-squares methods for solving partial differential equations. International Journal for Numerical Methods in Engineering, 10(5), 1976.

[28] FERNÁNDEZ-MÉNDEZ, S., HUERTA, A. Imposing essential boundary conditions in mesh-free methods. Computer Methods in Applied Mechanics and Engineering, 193(12-14), 2004.

[29] FERNÁNDEZ-MÉNDEZ, S., HUERTA, A., LIU, W. K. A comparison of two formulations to blend finite elements and mesh-free meth. Computer Methods in Applied Mechanics and Engineering, 193(12-14), 2004.

[30] HEGEN, D. Element-free Galerkin methods in combination with finite element approaches. Computer Methods in Applied Mechanics and Engineering, 135(1-2), 1996.

[31] HUERTA, A., BELYTSCHKO, T., FERNÁNDEZ-MÉNDEZ, S., RABEZUK. Meshfree methods. Encyclopedia of Computational Mechanics, vol. 1: Fundamentals. John Wiley \& Sons, 2004.

[32] IDELSON, S. R., OÑATE, E., CALVO, N., PIN, F. D. The meshless finite element method. International Journal for Numerical Methods in Engineering, 58(6), 2003.

[33] KALHEVIĆ, I., SAIGAL, S. An improved element free Galerkin formulation. International Journal for Numerical Methods in Engineering, 40(16), 1997. 
[34] KRYSL, P., BELYTSCHKO, T. Analysis of thin plates by the element-free Galerkin method, International Journal of Solids and Structures, 33(20-22), 1996.

[35] PIMENTA, P. M. Fundamentos da Mecânica das Estruturas. Texto do curso de Fundamentos da Teoria das Estruturas, Departamento de Estruturas e Fundações, Escola Politécnica da Universidade de São Paulo, 2004.

[36] PIMENTA, P. M., CAMPELLO, E. M. B., WRIGGERS, P. A triangular finite element applied to the geometrically exact analysis of elastic-plastic shells. Sixth World Congress on Computational Mechanics Second Asian-Pacific Congress on Computational Mechanics. Tsinghua University Press \& Springer-Verlag, Beijing, China, 2004.

[37] SIMO, J. C., FOX, D. D., HUGHES, T. J. R. Formulations of finite elasticity with independent rotations. Computer Methods in Applied Mechanics and Engineering, 95(2), 1992.

[38] TIAGO T. F., C. M., LEITÃO, V. M. A. Application of radial basis functions to linear and nonlinear structural analysis problems. Computers \& Mathematics with Applications, 51(8), 2006.

[39] WAGNER, G. J., LIU, W. K. Application of essential boundary conditions in meshfree methods: a corrected collocation method. International Journal for Numerical Methods in Engineering, 47(8), 2000.

[40] ZHU, T., ZHANG, J.-D., ATLURI, S. N. A local boundary integral equation (LBIE) method in computational mechanics, and a meshless discretization approach. Computational Mechanics, 21(3), 1998. 